\title{
Possibility and Evidence-Based Reliability Analysis and Design Optimization
}

\author{
Hong-Zhong Huang, Liping He, \\ Yu Liu, Ning-Cong Xiao, Yan-Feng Li and Zhonglai Wang \\ Department of Mechanical Engineering, \\ School of Mechanical, Electronic and Industrial Engineering, \\ University of Electronic Science and Technology of China, \\ No. 2006, Xiyuan Avenue, West Hi-Tech Zone, Chengdu, Sichuan, 611731, China
}

Received 2012-05-14; Revised 2012-07-30; Accepted 2013-05-03

\begin{abstract}
Engineering design under uncertainty has gained considerable attention in recent years. A great multitude of new design optimization methodologies and reliability analysis approaches are put forth with the aim of accommodating various uncertainties. Uncertainties in practical engineering applications are commonly classified into two categories, i.e., aleatory uncertainty and epistemic uncertainty. Aleatory uncertainty arises because of unpredictable variation in the performance and processes of systems, it is irreducible even adding more data or knowledge. On the other hand, epistemic uncertainty stems from lack of knowledge of the system due to limited data, measurement limitations, or simplified approximations in modeling system behavior and it can be reduced by obtaining more data or knowledge. More specifically, aleatory uncertainty is naturally represented by a statistical distribution and its associated parameters can be characterized by sufficient data. If, however, the data is limited and can be quantified in a statistical sense, epistemic uncertainty can be considered as an alternative tool in such a situation. Of the several optional treatments for epistemic uncertainty, possibility theory and evidence theory have proved to be the most computationally efficient and stable for reliability analysis and engineering design optimization. This study first attempts to provide a better understanding of uncertainty in engineering design by giving a comprehensive overview of its classifications, theories and design considerations. Then a review is conducted of general topics such as the foundations and applications of possibility theory and evidence theory. This overview includes the most recent results from theoretical research, computational developments and performance improvement of possibility theory and evidence theory with an emphasis on revealing the capability and characteristics of quantifying uncertainty from different perspectives. Possibility and evidence theory-based reliability methods have many advantages for practical engineering when compared with traditional probability-based reliability methods. They can work well under limited data while the latter need large amounts of information, more than possible in engineering practice due to aleatory and epistemic uncertainties. The possible directions for future work are summarized.
\end{abstract}

Keywords: Possibility Theory, Evidence Theory, Design Optimization, Per Formability Improvement, Various Uncertainties, Theoretical Research, Computational Development

\section{INTRODUCTION}

Uncertainty is one of the greatest challenges for engineering design (Huang et al., 2008; 2009; 2011a; 2011b; 2012a; Huang, 2012; Zhang et al., 2010a). Over the past two decades, there has been an ever-increasing tendency to take uncertainty analysis into account. Various uncertainties can be observed in engineering practices across multiple spatial and temporal scales, as well as phases of product design (Liu et al., 2009; 2012; Greene et al., 2011); these

\footnotetext{
Corresponding Author: Hong-Zhong Huang, Department of Mechanical Engineering, School of Mechanical, Electronic and Industrial Engineering, University of Electronic Science and Technology of China, No. 2006, Xiyuan Avenue, West Hi-Tech Zone, Chengdu, Sichuan, 611731, China
} 
must be carefully dealt with in engineering design activity. In fact, uncertainty is associated with both the qualitative and quantitative characteristics of design problems, hence, we first present classifications, theories and design considerations to provide a better understanding of uncertainty. A holistic review is then provided of alternative approaches to dealing with epistemic uncertainty and applying these approaches to design practices.

The characteristics of uncertainty depend on the mathematical theory within which problem situations are formalized (Klir, 1995). Generally speaking, each mathematical theory can characterize only some of the specific types of uncertainty. A more general theory can, of course, characterize more types of uncertainty. The theories and methods that we recommand will shed light on dealing with practical problems in engineering design.

\subsection{Definitions and Classifications of Uncertainty}

Different terms have been used as synonyms for uncertainty. For examples, words such as unsuredness, indefiniteness, unpredictability, indeterminacy, changeability, irregularity, arbitrariness, ambiguity, vagueness, randomness, variability and haphazardness provide various perspectives and/or specific nuances in meaning.

Uncertainty has several definitions. To some extent, it is associated with phenomena that are questionable, problematical, poorly defined or determined, lacking certain knowledge, or liable to change/vary (Klir and Folger, 1988). Uncertainty is also related to degree of belief in the validity of a particular proposition or datum (Agarwal et al., 2004; Kangas and Kangas, 2004). Based on the existing descriptions, Zimmermann (2000) gave a more general definition for uncertainty as follows.

Uncertainty implies that in a certain situation a person does not dispose about information which quantitatively and qualitatively is appropriate to describe, prescribe or predict deterministically and numerically a system, its behavior or other characteristics.

Uncertainty is also classified in several different ways by the literature. For example, Nikolaidis and Haftka (2001) reviewed the types of uncertainty involved in risk assessment problems and classified them into irreducible (random) and reducible uncertainty. The former is due to inherent randomness in physical phenomena or processes whereas the latter is due to a lack of knowledge. As their names imply, collecting data can reduce reducible uncertainty but not irreducible uncertainty. Oberkampf et al. (2000; 2004) considered a third type of uncertainty, namely error, which is defined as a recognizable deficiency in modeling and simulation that is not due to lack of knowledge.

When considering design problems, Robinson (1998) treated uncertainties using two alteratives, probabilistic and possibilistic methods, He pointed out that in exact system modeling, probabilistic techniques are characterized by random variables describing the various sources of uncertainties; these often referred to as reliability methods by structural engineers, are typically applied to systems of small or moderate complexity. In contrast, possibilistic techniques (often referred to as the fuzzy set theory or possibility theory) are typically applied to large and complex systems.

Zimmermann (2000) classified the causes of uncertainty (not types of uncertainty, as he emphasized) as: lack of information, abundance of information (complexity), conflicting evidence, ambiguity, error in measurement and subjective belief. Rowe (1994), on the other hand, classified uncertainty as metrical (measurement variability and uncertainty), structural (uncertainty due to the complexity of systems), temporal (uncertainty about future and past states of nature) and translational (uncertainty in explaining uncertain results). Ferson and Ginzburg (1996) used only two broad classes, namely ignorance and variability, where variability includes the variation between individuals and spatial and temporal variation. Ignorance refers to uncertainty due to lack of knowledge; the true value exists and it is fixed, but we do not know it. According to this definition, ignorance can be reduced by further study better measurement techniques and so on. Variability, on the other hand, remains the same no matter how many additional studies are conducted.

The three distinct categories of variability, uncertainty and error, proposed by Moens and Vandepitte (2004), which have been popularly accepted widely and used in modeling, are similar to classifications by Oberkampf et al. (2004) and Agarwal et al. (2004). It is noteworthy that the word uncertainty often refers to random variability. A distinction can also be made between aleatory uncertainty (referred to as variability, irreducible uncertainty, inherent uncertainty and stochastic uncertainty), epistemic uncertainty (referred to as reducible uncertainty, subjective uncertainty, state-of-knowledge uncertainty, model form uncertainty and simple uncertainty) and error (Oberkampf et al., 2000; 2004). Aleatory uncertainty regarding a quantity can often be distinguished from other types of uncertainty by its characterization as a random value with known distribution (Oberkampf et al., 2004). These classifications can be illustrated with their causations as shown in Fig. 1.

In view of descriptions of degrees of uncertainty and simplifications of systems, Klir and Folger (1988) and Klir and Yuan (1995) first reviewed various meanings of the word "uncertain", then categorized uncertainty naturally into vagueness and ambiguity. 


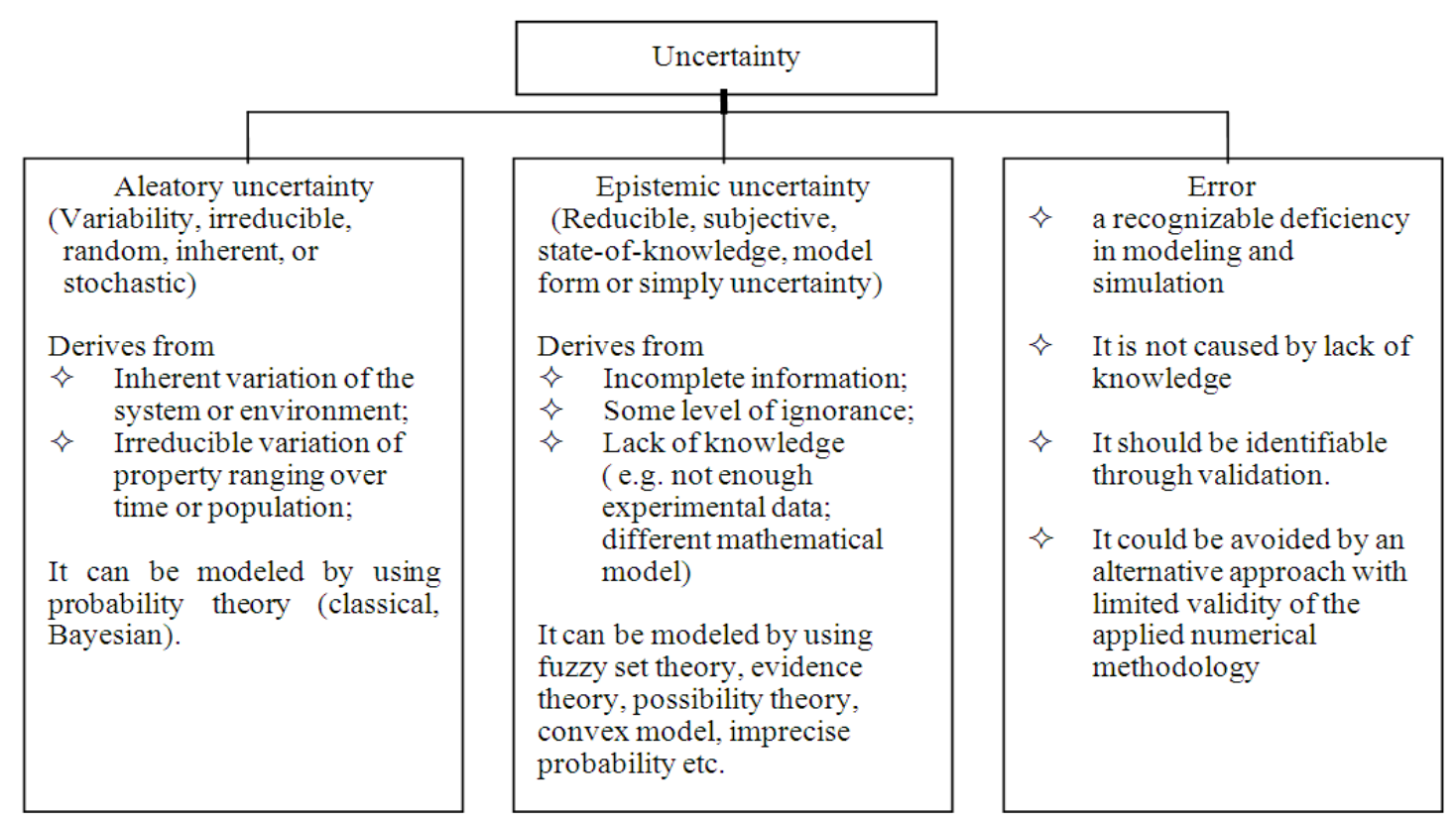

Fig. 1. A well-known classification of uncertainty (Oberkampf et al., 2000; 2004; Moens and Vandepitte, 2004)

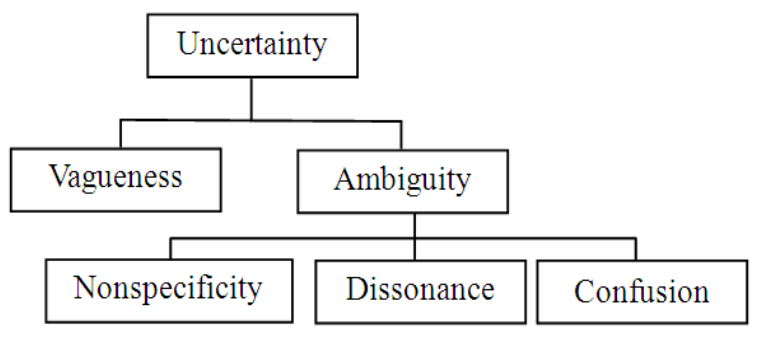

Fig. 2. Klir's classification of uncertainty (Klir and Folger, 1988; Klir and Yuan, 1995)

Generally speaking, vagueness reveals the difficulty of making sharp or precise distinctions in the world and ambiguity, arises out of one-to-many relations. Klir and Folger (1988) also put forth a set of similar concepts for these two distinct forms of uncertainty; that is, vagueness is connected with such concepts as fuzziness, haziness, cloudiness, unclearness, indistinctiveness and shaplessness, whereas ambiguity is connected with such concepts as nonspecificity, one-to-many relations, variety, generality, diversity and divergence.

Klir's classifications of uncertainty can be seen in Fig. 2. There the word vagueness has the same meaning as fuzziness and the word dissonance is sometimes replaced by conflict. Klir's purpose was to provide a basic framework for characterizing the full scope of the concept of uncertainty and its relationship to the increasingly important concepts of information and complexity; his work plays a fundamental role in the relevant theories on uncertainty and information.

\subsection{Sources of Uncertainty}

In order to develop a general methodology for quantifying various types of uncertainty, the vital and necessary first step is identifying of the sources of uncertainty. In different phases of modeling and simulation, uncertainty arises from the following (Robinson, 1998; Agarwal et al., 2004; Huang et al., 2004; 2006c; 2009; 2012b):

- External system parameters (load, temperature, radiation)

- Internal system parameters (material properties)

- Modeling of the physical system (conceptual or mathematical methods)

- Observational uncertainty

- Solution processes of the mathematical model (numerical or algorithmic uncertainty)

- Representation of the numerical solution

- Field data or experimental data

\subsection{Theories of Uncertainty Modeling}

There is an abundant collection of theories regarding modeling all types of uncertainties. 


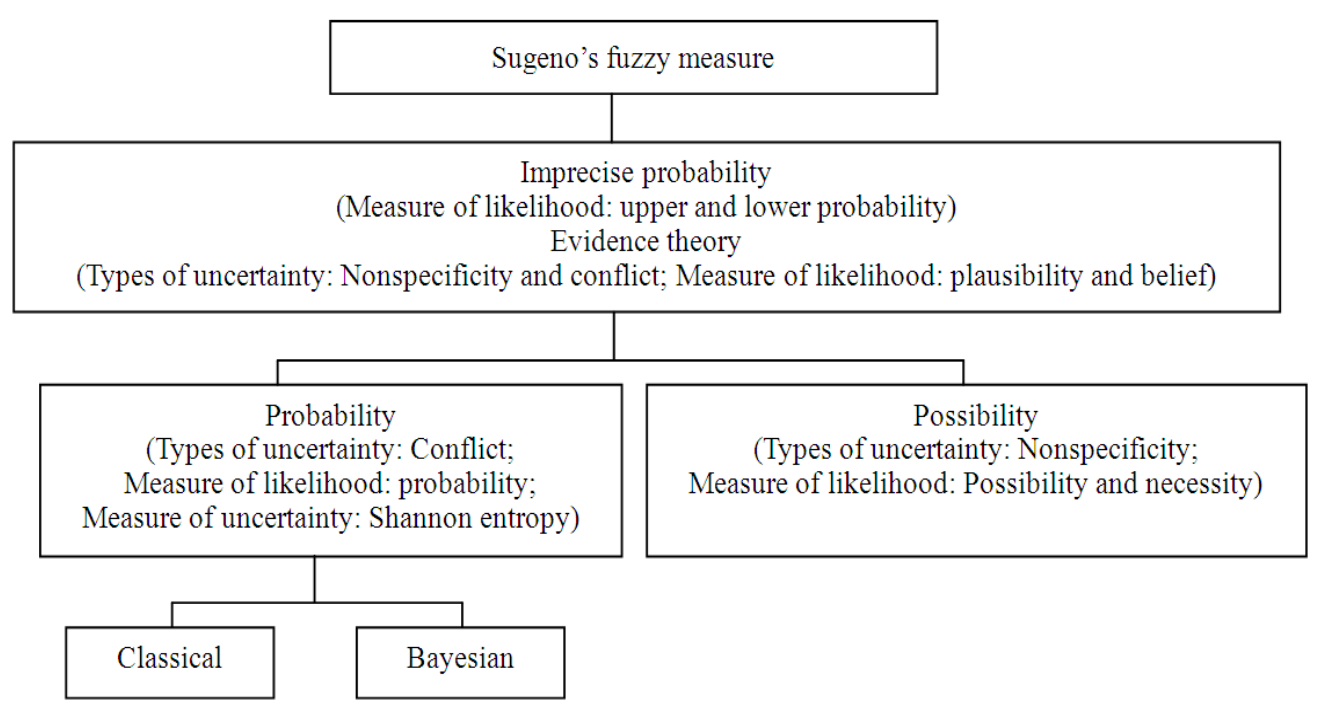

Fig. 3. Families of theories of uncertainty (Nikolaidis and Haftka, 2001)

Before fuzzy measure was proposed by Sugeno, probability theory (including classical or frequentist) was the dominant and most effective way to model uncertainty, especially stochastic uncertainty when sufficient information is available. This theory is not appropriate for reducible uncertainty and error, however, because the additivity axioms, which probability theory relies on, are unable to express lack of knowledge in scarce-data situations. For this reason, alternative uncertainty analysis tools such as imprecise probability and evidence theory, have been developed; they can be combined with probability theory to develop a framework in a specific field (Nikolaidis and Haftka, 2001), e.g., risk assessment of systems when data is scarce (Nikolaidis and Haftka, 2001). Such a family of theories of uncertainty is presented in Fig. 3.

We need to note that these theories of imprecise probability (i.e., intervals of probabilities) and evidence theory don't conflict with Bayesian or classical probability. Instead, they are tools that complement probabilistic methods for problems which probability theory cannot solve (Moller et al., 2006). These theories are flexible enough to model both nonspecificity and conflict types of uncertainty (see classification in Fig. 2). The fundamental measure in the most general theory is Sugeno's fuzzy measure, which is less restrictive than measures in the other two theories of probability and possibility.

\subsection{Uncertainty Measures}

After Klir overviews the various types of uncertainty, he discusses their relation to information and complexity and investigates in detail measures of the individual types of uncertainty (Klir and Folger, 1988;
Klir, 1995; Klir and Yuan, 1995). Measures of types of uncertainty must be formulated in accordance with their own distinct framework. The understanding that measures are related to types of uncertainty has been widely accepted. The formulas are as shown in Table 1.

\subsection{Design Under Uncertainty}

Because nature doesn't adhere to determinism, the development of uncertainty analysis in engineering science has received increased attention, from both the epistemological and methodological perspectives. It is generally believed that unless the impact of uncertainties is considered, a design solution may be sensitive to variations in input which will lead to a loss of system performance, or to a potential risk of violating critical design constraints (Du and Chen, 2004). As a result, design under uncertainty has been applied increasingly in practice.

The characteristics and formulations of uncertainty have to be mathematically quantified before design optimization is conducted. A real design or decision problem involving uncertainty may be formalized in all theories of uncertainty. Each is a certain mathematical model of the specific situation; for example, as depicted in Fig. 3, probability theory can model decision situations in terms of conflicting degrees of belief which are mutually exclusive. On the other hand, possibility theory can model a decision situation in terms of conflict-free, or nonspecificity degrees of belief which are presented as nested subsets of alternatives (Klir, 1995; Huang, 1997). Moreover, each method of handling uncertainty emphasizes a different paradigm. 
Table 1. A summary of measures of uncertainty by Klir and Folger (1988)

\begin{tabular}{llll}
\hline Type & Name & Formula & Notation \\
\hline \multirow{2}{*}{ Classical } & Hartley information & $\mathrm{I}(\mathrm{N})=\log _{2} \mathrm{~N}$ & $\begin{array}{l}\text { N: cardinality of a crisp set } \\
\text { P: Probability distribution }\end{array}$ \\
& Shannon entropy & $\mathrm{H}(\mathrm{P})=-\sum_{\mathrm{i}=1}^{\mathrm{n}} \mathrm{p}_{\mathrm{i}} \log _{2} \mathrm{p}_{\mathrm{i}}$ & $\mathrm{P}=\left(\mathrm{p}_{1}, \mathrm{p}_{2}, \ldots, \mathrm{p}_{\mathrm{n}}\right) \Pi$ : possibility distribution \\
General & $U$-uncertainty & $\mathrm{U}(\Pi)=\sum_{\mathrm{i}=1}^{\mathrm{n}}\left(\pi_{\mathrm{i}}-\pi_{\mathrm{i}+1}\right) \log _{2} \mathrm{i}$ & $\begin{array}{l}\Pi=(123) \text { membership function } \mu \mathrm{A}(\mathrm{x}) \text { : } \\
\text { (or possibility distribution) } \mathrm{C}: \text { fuzzy complement }\end{array}$ \\
Vagueness & Measure of fuzziness & $\mathrm{f}_{\mathrm{C}}(\mathrm{A})=|\mathrm{X}|-\sum_{\mathrm{x} \in \mathrm{X}}\left|\mu_{\mathrm{A}}(\mathrm{x})-\mathrm{C}\left(\mu_{\mathrm{A}}(\mathrm{x})\right)\right|$ & m: basic assignment \\
& Measure of onspecificity & $\mathrm{V}(\mathrm{m})=\sum_{\mathrm{A} \in \mathbb{F}} \mathrm{m}(\mathrm{A}) \log _{2}|\mathrm{~A}|$ & $\mathrm{F}$ : set of focal element F: set of focal element \\
Ambiguity & Measure of dissonance & $\mathrm{E}(\mathrm{m})=-\sum_{\mathrm{A} \in \mathbb{F}} \mathrm{m}(\mathrm{A}) \log _{2} \mathrm{Pl}(\mathrm{A})$ & \\
& Measure of confusion & $\mathrm{C}(\mathrm{m})=-\sum_{\mathrm{A} \in \mathbb{F}} \mathrm{m}(\mathrm{A}) \log _{2} \mathrm{Bel}(\mathrm{A})$ & \\
\hline
\end{tabular}

For example, robust design is expected to improve the quality of a product by minimizing the effects of input variation, whereas a reliability-based design approach focuses on maintaining design feasibility at expected probabilistic levels (Huang, 1995; 1996; Tu and Choi, 1999).

The traditional way of defining design problems, such as optimization problems, is to create a model of the system that assumes that it is exact and deterministic. Recently, a number of non-deterministic approaches for design problems have emerged, mainly in response to criticism of the credibility of standard probabilistic analysis which ignores information on epistemic uncertainty (Huang et al., 2008; 2009; 2012a). Adding non-probabilistic methods to traditional design methods makes it more difficult for a designer to choose the best method to use. The common non-probabilistic techniques used to model uncertainties include possibility theory and evidence theory, which are the main tools to be discussed in the rest of this study.

The aim of this section is to present a holistic view on design optimization under uncertainty in the context of possibility theory and evidence theory when data is insufficient.

\section{THEORETICAL FOUNDATIONS OF POSSIBILITY THEORY AND EVIDENCE THEORY}

\subsection{Possibility Theory}

Possibility theory was formulated by Zadeh (1978). As one of three constituents of fuzzy theory (the others are fuzzy set theory and fuzzy logic) (Klir, 2000), possibility theory provides a theoretical framework for practical applications of fuzzy theory. Zadeh (1978) points out in his paper that, much of the information on which decisions are based more possibilistic rather than probabilistic in nature. Based on this premise, Zadeh proposes the theory of possibility, analogous to but different from probability theory, to express the intrinsic fuzziness of natural language and uncertainty information. Zadeh focuses on information's meaning, rather than its measure. He provides a set of ways of analyzing and translating propositions expressed in natural language by computing the possibility distribution of a set of fuzzy relations.

As noted by Dubois and Prade $(1983 ; 1988)$, the original goal of possibility theory is finding a mathematical tool for further studying fuzzy language and approximate reasoning, thus extending and systematizing possibility theory. Klir and Folger (1988); Klir (1995) and Klir and Yuan (1995) concluded that possibility theory emerged as a natural tool for modeling and handling uncertainty involving knowledge expressed in natural language and represented by fuzzy propositions. Kaufmann (1983) thought that the role possibility theory plays for fuzzy sets is analogous to that mathematical expectancy plays in probability theory and that such valuation agrees with information available subjectively.

In general, possibility theory is one of several formal mathematical systems suitable for characterizing and analyzing situations that involve various types of uncertainties (Klir, 2000). Due to practical demands, a variety of interpretations of each of these formal systems for possibility theory have been studied, in correspondence with diverse types of uncertainty. A representative but not total list is the possibilistic interpretation by Dubois and Prade (1983), the modal logic interpretation by Klir (2000), the DST (or evidence theory) interpretation examined and compared by Sudkamp (1992) and Klir and Yuan (1995) and the fuzzy-set interpretation by Zadeh (1978) and Klir (referred to as standard form later as and revised form). 


\subsubsection{Definition of Possibility}

Possibility is a subjective measure that expresses the extent to which, either, a person thinks that an event can occur or, alternatively, the available evidence shows that an event will occur (De-Cooman, 1997; Nikolaidis and Haftka, 2001).

In 1949, Shackle was convinced of the need for a formal mathematical system in terms of possibility theory in economics. He defined possibility, in 1961, as the degree to which it is likely for an event to occur. Moreover, he stated that possibility should be used instead of probability when the conditions under which we have to make a decision under uncertainty cannot be reproduced (Liu et al., 2009).

The term possibilistic first occurred in the study on possible automata presented by (Gaines and Kohout, 1975), but it is Zadeh who coined the concepts of possibility measure, possibility distribution and possibility theory, in which the definition of possibility is quite different from that of modal logic (Zadeh, 1978; Klir, 2000). According to Zadeh, a proposition that associates an uncertain quantity with a fuzzy set induces a possibility distribution for this quantity which provides information about the values this quantity can assume.

Another interpretation, which is based on evidence theory, denotes that possibility is the limit of plausibility for a body of evidence that is nested (Shafer, 1976; Klir and Yuan, 1995).

Possibility is also viewed as an upper bound of probability (Zadeh, 1978; Klir, 2000). Giles (1982) a definition of possibility according to which the possibility of an event is the smallest amount in an interval of $[0,1]$.

\subsubsection{Standard Fuzzy-Set Interpretation of Possibility Theory}

Among all the multifarious interpretations of possibility theory, the well-known fuzzy-set interpretation has proved to be the most prominent and useful. This is mainly because fuzzy set is widely used and possibility theory palys an important role in approximate reasoning.

Let $\mathrm{X}$ denote a variable that takes values in a universe of discourse, $U$ and $F$ is a fuzzy set on $U$, whose membership function, $\mu_{\mathrm{F}}(\mathrm{u})$, shows the compatibility of an assigned value, $\mathrm{u}$, from $\mathrm{X}$ with the concept of $\mathrm{F}$. If $\mathrm{F}$ acts as an elastic constraint on possible values that may be assigned to $\mathrm{X}$, then $\mathrm{F}$ is a fuzzy restriction on $\mathrm{X}$ (or associated with $\mathrm{X}$ ); it is referred to as $\mathrm{R}(\mathrm{X})$. A fuzzy proposition, " $\mathrm{X}$ is F", can be described as Equation 1:

$$
\mathrm{R}(\mathrm{X})=\mathrm{F}
$$

In the view of the possibility hypothesis, there is no other information regarding $\mathrm{X}$ except the proposition " $\mathrm{X}$ is F". Thus the proposition associates a possibility distribution, $\Pi_{X}$, with $X$ which is postulated to be equal to $\mathrm{R}(\mathrm{X})$, i.e., $\Pi_{\mathrm{X}}$ can be expressed as Equation 2:

$\Pi_{\mathrm{X}}=\mathrm{R}(\mathrm{X})$

Correspondingly, let $\pi_{\mathrm{X}}$ denote the possibility distribution function associated with $\mathrm{X}$ (or the possibility distribution function of $\Pi_{X}$ ), then the degree of possibility of $\mathrm{X}=\mathrm{u}$ for all $\mathrm{u} \in \mathrm{U}$, is defined as numerically equal to the degree of membership function when $\mathrm{X}=\mathrm{u}$. Formally:

$\forall \mathrm{u} \in \mathrm{U}, \quad \pi_{\mathrm{X}}(\mathrm{u})=\mu_{\mathrm{F}}(\mathrm{u})$

Equation 3 formulates the connection between membership degrees and possibility degrees, in a more general framework, by the proposition " $\mathrm{X}$ is F".

Let $\Pi_{X}$ be the possibility distribution associated with a variable, $X$, which takes value in $\mathrm{U}$. Then the possibility measure, $\pi(\mathrm{A})$, is defined as a number in $[0$, 1]. When $A$ is a nonfuzzy (crisp) subset of $U$, we have:

$\mathrm{P}_{\mathrm{oss}}\{\mathrm{X} \in \mathrm{A}\} \equiv \pi(\mathrm{A}) \equiv \sup _{\mathrm{u} \in \mathrm{A}} \pi_{\mathrm{X}}(\mathrm{u})$

When A is a fuzzy subset of $U$, a more general definition of possibility measure is as follows:

$\mathrm{P}_{\text {oss }}\{\mathrm{X}$ is $\mathrm{A}\} \equiv \pi(\mathrm{A}) \equiv \sup _{\mathrm{u} \in \mathrm{U}}\left(\mu_{\mathrm{A}}(\mathrm{u}) \wedge \pi_{\mathrm{X}}(\mathrm{u})\right)$

Let A and B be arbitrary fuzzy subsets of $U$, the following Equation 6 and 7 are induced from Equation 4 and (5):

$\pi(\mathrm{A} \cup \mathrm{B})=\pi(\mathrm{A}) \vee \pi(\mathrm{B})$

It can be derived that, $\max (\pi(\mathrm{A}), \pi(\overline{\mathrm{A}}))=1$ :

$\pi(\mathrm{A} \cap \mathrm{B}) \leq \pi(\mathrm{A}) \wedge \pi(\mathrm{B})$

Zadeh's primary contribution is that he introduces a meaningful connection between fuzzy propositions and possibility measures, a connection that has been widely adopted in literature as the standard fuzzy-set 
interpretation of possibility theory. As argued in his paper, by employing the concept of a possibility distribution, it is possible to translate a proposition expressed in natural language into a procedure. This manipulates the probability distribution using the combination rules of fuzzy sets and particularly of fuzzy restrictions (Dubois and Prade, 1980). In this study, Zadeh did not, however, address the connection between possibility measures and dual necessity measures; that work was done later.

As seen in the above interpretation, we can conclude that interpretations such as the initial fuzzy set (Zadeh, 1978) occurred naturally because of the similarity between the mathematical structures of possibility measures and fuzzy sets. In possibility theory, the underlying families of nested sets comprise focal element, whereas in fuzzy sets, these families comprise $\alpha$-cuts (Klir, 2000).

\subsubsection{Some Extensions on the Standard Interpretation}

Dubois and Prade (1988) gave a more detailed interpretation of possibility theory. De-Cooman (1997) systemized the existing views), especially with regard to uncertain measures. They pointed out that possibility theory denotes uncertainty of a proposition by means of a pair of fuzzy measures, i.e., possibility measure, $\mathrm{P}_{\text {oss }}$ (A) and necessity measure, $\mathrm{N}_{\mathrm{ec}}$ (A). Possibility theory might be characterized in terms of either of these measures $\left(\mathrm{P}_{\text {oss }}(\mathrm{A}), \mathrm{P}_{\text {oss }}(\overline{\mathrm{A}})\right)$, or expressed as $\left(\mathrm{P}_{\mathrm{oss}}(\mathrm{A}), \mathrm{N}_{\mathrm{ec}}(\mathrm{A})\right)$ equivalently.

Necessity measure is defined, as being one of the two dual formulations, in the sense that can be expressed as Equation 8:

$$
\mathrm{N}_{\mathrm{ec}}(\mathrm{A})=1-\mathrm{P}_{\mathrm{oss}}(\overline{\mathrm{A}})
$$

Analogous to the additive axiom of probability measure, possibility measure satisfies the following axiomatic requirement, which is expressed as Equation 9:

$$
\mathrm{P}_{\text {oss }}(\mathrm{A} \cup \mathrm{B})=\max \left(\mathrm{P}_{\text {oss }}(\mathrm{A}), \mathrm{P}_{\text {oss }}(\mathrm{B})\right)
$$

Some basic properties in Equation 10-13 of the two fuzzy measures can be induced (Dubois and Prade, 1988; Klir and Yuan, 1995; De-Cooman, 1997):

$$
\begin{aligned}
& \mathrm{N}_{\mathrm{ec}}(\mathrm{A} \cap \mathrm{B})=\min \left(\mathrm{N}_{\mathrm{ec}}(\mathrm{A}), \mathrm{N}_{\mathrm{ec}}(\mathrm{B})\right) \\
& \max \left(\mathrm{P}_{\text {oss }}(\mathrm{A}), \mathrm{P}_{\text {oss }}(\overline{\mathrm{A}})\right)=1
\end{aligned}
$$

$$
\begin{aligned}
& \min \left(\mathrm{N}_{\mathrm{ec}}(\mathrm{A}), \mathrm{N}_{\mathrm{ec}}(\overline{\mathrm{A}})\right)=0 \\
& \mathrm{P}_{\mathrm{oss}}(\mathrm{A}) \geq \mathrm{N}_{\mathrm{ec}}(\mathrm{A})
\end{aligned}
$$

\subsubsection{Revised Fuzzy-Set Interpretation of Possibility Theory}

After introducing the most common axiomatic characterizations of possibility theory and some basic properties of possibility measure, we'd like to summarize a revised fuzzy-set interpretation of possibility theory, proposed to overcome the difficulties of the standard interpretation when applied to subnormal fuzzy sets (i.e., height of a fuzzy set F, $\left.\mathrm{h}_{\mathrm{F}}=\sup \mu_{\mathrm{F}(\mathrm{u})^{11}}\right)$, as discussed by Klir (2000).

û̂U

The difficulty with subnormal fuzzy sets was first recognized by Yager (1986), who demonstrated that the standard interpretation expressed by Equation 3 is not coherent when $\mathrm{F}$ is subnormal, it proved to be one of the key properties of possibility theory, as expressed by inequality Equation 13, that does not come into existence when $\mathrm{h}_{\mathrm{F}}<1$.

In order to overcome the defect, Yager proposed a new function, called a measure of certainty, to take the place of the necessity function, which is given by Equation 14:

$\mathrm{C}_{\text {ert }}(\mathrm{A})=\min \left(\mathrm{P}_{\text {oss }}(\mathrm{A}), \mathrm{N}_{\mathrm{ec}}(\mathrm{A})\right)$

Dubois and Prade (1987) pointed out that such replacement violates Equation 10, one of the basic requirements of possibility theory. As a result, Dubois and Prade (1987) suggested keeping the necessity function but replacing Equation 8 with a generalized equation, which is expressed as Equation 15:

$\mathrm{N}_{\mathrm{ec}}(\mathrm{A})=\mathrm{h}_{\mathrm{F}}-\mathrm{P}_{\mathrm{oss}}(\overline{\mathrm{A}})$

This is then converted to Equation 8 when $\mathrm{F}$ is normal and satisfies both Equation 10 and 13 for any subsets of $U$, hence it sounds more reasonable.

To address the still-existing severe deficiencies argued by Klir (2000), they modified possibility theory to adapt to subnormal fuzzy sets by replacing Equation 4, 8 and 9 respectively. This can be seen in their paper (Klir, 2000 ), with the complementary case in which $\mathrm{A}=\mathrm{U}$ or $\bigcup_{i \in I} A_{i}=U$, where $I$ is an arbitrary index set.

Obviously, all of the above previous work is more an interpretation of systems that are based on a given modification of possibility theory, rather than an essential fuzzy-set interpretation of possibility theory. 
Table 2. The revised fuzzy-set interpretation of possibility theory proposed by Klir (2000)

\begin{tabular}{ll}
\hline Standard Interpretation & Revised interpretation \\
\hline$\pi_{\mathrm{X}}(\mathrm{u})=\mu_{\mathrm{F}}(\mathrm{u})$ & $\pi_{\mathrm{X}}(\mathrm{u})=\mu_{\mathrm{F}}(\mathrm{u})+\mathrm{c}_{\mathrm{F}}$ \\
& $=\mu_{\mathrm{F}}(\mathrm{u})+1-\mathrm{h}_{\mathrm{F}}$ \\
$\mathrm{m}_{\mathrm{F}}(\mathrm{u})=\inf _{\mathrm{u} \in \mathrm{U}} \mu_{\mathrm{F}}(\mathrm{u})$ & $\mathrm{m}_{\mathrm{F}}(\mathrm{u})=\inf _{\mathrm{u} \in \mathrm{U}} \mu_{\mathrm{F}}(\mathrm{u})+1-\mathrm{h}_{\mathrm{F}}$ \\
$\sum_{\mathrm{A} \in \mathrm{P}(\mathrm{x})} \mathrm{m}_{\mathrm{F}}(\mathrm{A})=\mathrm{h}_{\mathrm{F}}<1$ & $\sum_{\mathrm{A} \in \mathrm{P}(\mathrm{x})} \mathrm{m}_{\mathrm{F}}(\mathrm{A})=1$ \\
$\mathrm{P}_{\mathrm{oss}}(\mathrm{A}) \equiv \sup _{\mathrm{u} \in \mathrm{U}} \min \left(\mu_{\mathrm{F}}(\mathrm{u}), \mu_{\mathrm{A}}(\mathrm{u})\right)$ & $\mathrm{P}_{\mathrm{oss}}(\mathrm{A}) \equiv \sup _{\mathrm{u} \in \mathrm{U}} \mathrm{min}$ \\
& $\left(\mu_{\mathrm{F}}(\mathrm{u})+1-\mathrm{h}_{\mathrm{F}}, \mu_{\mathrm{A}}(\mathrm{u})\right)$ \\
\hline
\end{tabular}

When study returns to fuzzy-set interpretation, the possibility distribution function, $\pi(\mathrm{u})$, satisfies the following equation for subnormal fuzzy set, which is expressed as Equation 16:

$$
\sup _{u \in U} \pi(u)=1
$$

To consider the connection between possibility theory and evidence theory, Klir (2000) revised some definitions as in Table 2.

where function, $\mathrm{m}$, is called a basic probability assignment function in evidence theory and $c_{F}$ is a constant for each given fuzzy set, F. When Equation 16 is satisfied, then $c_{F}=1-h_{F}$.

Klir's direction for improving the initial interpretation of possibility theory can thus be summarized briefly as:

- Keep coherent all fuzzy sets, regardless of whether they are normal or not, so they violate no property of possibility theory

- Capture the evidence expressed by any given fuzzy proposition, $\mathrm{m}_{\mathrm{F}}$, which carries information in the framework of evidence theory

- Be meaningful on intuitive grounds

\subsection{Evidence Theory}

The origins of evidence theory, also called Dempster-Shafer Theory (DST), can be traced back to the work by Dempster (1967) which developed a system of upper and lower probabilities that do not satisfy additivity. Following Dempster's work, it was his student, Shafer (1976) and Liu et al. (2009) who extended Dempster's probability to the theory of evidence in 1976, including a more thorough explanation of belief functions. The name "Dempster-Shafer theory" was coined by Barnett in a paper which marked the entry of the belief functions into the field of artificial intelligence (Aughenbaugh and Paredis, 2005).
Evidence theory could be viewed as a branch of mathematics which studies empirical evidence in order to construct a coherent picture of reality (Fioretti, 2004). It can narrow down a hypothesis set with the accumulation of evidence and it allows for a representation of ignorance due to uncertainty in the evidence (Bhattacharya, 2000). When ignorance has the value of zero, the Dempster-Shafer model is reduced to the standard Bayesian model. Thus, the Dempster-Shafer theory is an attempt to generalize probability theory by introducing a rule for combining distinct bodies of evidence (Beynon et al., 2000); it is thus actually a numerical method of evidential reasoning. Compared with Bayesian theory, evidence theory feels closer to our human perception and reasoning processes. Its ability to assign uncertainty or ignorance to propositions is a powerful tool for dealing with a large range of problems that otherwise would seem intractable (Wu et al., 2002).

There have been many interpretations of the Dempster-Shafer theory, (Jumarie, 1994; Kohlas and Monney, 1994; Rowe, 1994; Utkin, 1994; Wang, 1994; Wonneberger, 1994; Yager et al., 1994; Cai et al., 1995a; 1995b) including probabilistic approaches and nonprobabilistic ones. Also, there have been many closely related developments in recent years. The most influential version of the theory is still Shafer's presentation in his book A Mathematical Theory of Evidence (Shafer, 1976), which we follow in providing a brief introduction of evidence theory.

Evidence theory starts with defining a frame of discernment that is a set of mutually exclusive "elementary" propositions; it can be viewed as a finite sample space in probability theory. Evidence theory uses two measures, Belief (Bel) and Plausibility (Pl), which are used to characterize uncertainty. In the DempsterShafer theory, evidence is represented by the basic probability assignment and the combination rule of evidence is discussed.

\subsubsection{Basic Concepts of Evidence Theory}

Liu et al. (2009); Klir (1995) and Shafer (1976) let $\mathrm{U}$ denote a finite, nonempty universal set that represents the entire collection of elements having the same characteristics, which is usually called a Frame of Discernment (FD) in DS theory. Let $\wp(\mathrm{U})$ denote the power set of U, set A is a collection of some elements of U. Then, available evidence can be expressed with respect to the nonnegative function as Equation (17) and (18):

$$
\mathrm{m}: \wp(\mathrm{U}) \rightarrow[0,1]
$$


Such that $\mathrm{m}(\varnothing)=0$ and:

$\sum_{\mathrm{A} \in \mathfrak{\wp}(\mathrm{U})} \mathrm{m}(\mathrm{A})=1$

This function, $\mathrm{m}$, is called a Basic Probability Assignment (BPA) (or mass function). For each set, $\mathrm{A} \in$ $\wp(\mathrm{U})$, the function value, $\mathrm{m}(\mathrm{A})$, measures the degree of evidence supporting the claim that a specific element of $\mathrm{U}$ belongs to set $\mathrm{A}$, as well as the degree to which we believe that such a claim is warranted (Klir, 1995).

Given a basic probability assignment, $\mathrm{m}$, each set, $\mathrm{A}$ $\in \wp(\mathrm{U})$, for which $\mathrm{m}(\mathrm{A}) \neq 0$, is called a focal element and the value, $\mathrm{m}(\mathrm{A})$, is called the weight of $\mathrm{A}$. The family, $\mathbb{F}$, of all the focal elements of $m$ characterizes the subsets of the frame of discernment on which all the available evidence rests. The pair $(\mathbb{F}, \mathrm{m})$ is called a body of evidence (or belief structure) denoted by $\mathrm{m}$.

\subsubsection{Belief and Plausibility Measures}

Two large classes of fuzzy measures, referred to as the belief measure and plausibility measure, respectively, characterize the mathematical theory of evidence. Given a basic probability assignment, $\mathrm{m}$, the two measures are correspondingly defined by the Equation 19 and 20:

$$
\begin{aligned}
& \operatorname{Bel}(A)=\sum_{B \subseteq A} m(B) \\
& \operatorname{Pl}(A)=\sum_{B \cap A \neq \varnothing} m(B)
\end{aligned}
$$

They show that Bel and Pl give the lower and upper bounds of the event, respectively. They are mutually dual in the sense that one of them can be uniquely transformed by the other, as seen in the Equation 21:

$$
\operatorname{Pl}(\mathrm{A})=1-\operatorname{Bel}(\overline{\mathrm{A}})
$$

where, $\bar{A}$ is the classical complement of $A$. This definition reflects the fact that all basic assignments must sum to 1, as seen in Equation 15.

An inverse procedure is also possible for all $\mathrm{A} \in$ $\wp(\mathrm{U})$, e.g., (Klir, 1995) Equation 22:

$$
\mathrm{m}(\mathrm{A})=\sum_{\mathrm{B} \subseteq \mathrm{A}}(-1)^{|\mathrm{A}-\mathrm{B}|} \operatorname{Bel}(\mathrm{B})
$$

Belief and plausibility measures satisfy both the axioms of fuzzy measures (Klir and Yuan, 1995) and the following additional axioms in Equation 23 and 24:

$\operatorname{Bel}\left(A_{1} \cup A_{2}\right) \geq \operatorname{Bel}\left(A_{1}\right)+\operatorname{Bel}\left(A_{2}\right)-\operatorname{Bel}\left(A_{1} \cap A_{2}\right)$

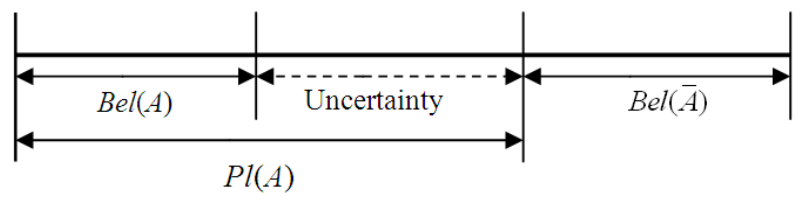

Fig. 4. Relation of belief measure and plausibility measure

$\operatorname{Pl}\left(\mathrm{A}_{1} \cap \mathrm{A}_{2}\right) \leq \operatorname{Pl}\left(\mathrm{A}_{1}\right)+\operatorname{Pl}\left(\mathrm{A}_{2}\right)-\operatorname{Pl}\left(\mathrm{A}_{1} \cup \mathrm{A}_{2}\right)$

and then the following properties can be derived Equation 25:

$$
\begin{aligned}
& \operatorname{Bel}(\mathrm{A})+\operatorname{Bel}(\overline{\mathrm{A}}) \leq 1 \\
& \operatorname{Pl}(\mathrm{A})+\operatorname{Pl}(\overline{\mathrm{A}}) \geq 1
\end{aligned}
$$

Bel(A) in Equation 26 represents the total evidence or belief that the elements belong to A. The total evidence or belief, $\mathrm{Pl}(\mathrm{A})$ in Equation 27, represents, moreover, the additional evidence or belief corresponding to the focal elements overlapping with A (Henkind and Harrison, 1988; Klir and Parviz, 1992). Thus, the relation between the two dual measures is:

$$
\operatorname{Pl}(\mathrm{A}) \geq \operatorname{Bel}(\mathrm{A})
$$

Equation 18 and 27 can be visualized by Fig. 4 (Klir and Parviz, 1992).

\subsubsection{Postulates}

The following postulates are assumed, which shape the foundation of evidence theory (Beynon et al., 2000) in Shafer's interpretation (Shafer, 1976):

Postulate $1=$ Chance is the limit of the proportion of positive outcomes among all outcomes

Postulate 2 = Chances, if known, should be used as belief functions

Postulate 3 = Evidence combination refers to the pooling, or accumulating, of distinct bodies of evidence

Postulate $4=$ Dempster's rule can be used on belief functions for evidence combination

\subsubsection{Bodies of Evidence}

\subsubsection{Algebraic interpretation (Kohlas and Monney, 1994)}

Evidence theory is connected with the description and analysis of possibly incomplete and uncertain information relative to a certain precise question. 
In an abstract setting, we call two sets, $\mathrm{H}$ and $\mathrm{A}$, of elements the hypotheses and argument, respectively. Then the triplet $(\mathrm{H}, \mathrm{A}, \mathrm{s})$ where $\mathrm{s}$ is an allocation of support is called a body of arguments. This simple algebraic structure acts as the foundation for evidence theory.

In the body of arguments, (H, A, s), not all arguments in $A$ may be equally likely. Some may be more probable than others. Thus, some hypotheses may become more credible than others, depending on the likelihood of the arguments supporting them.

The likelihood of arguments can be measured by probabilities, however, the Boolean algebra, A, may be too large to associate a probability with every element. As is usual in probability theory we consider a sub- $\sigma$ algebra, $\mathrm{A}_{0}$ contained in $\mathrm{A}$ and associate a probability, $\mathrm{P}$ $(\alpha)$, with every element, $\alpha$, of $A_{0}$. Thus $P(\alpha)$ is a probability measure on $\mathrm{A}_{0}$. The quintuple $\left(\mathrm{H}, \mathrm{A}, \mathrm{A}_{0}, \mathrm{P}, \mathrm{s}\right)$ is called a body of evidence.

\subsubsection{Axiomatic Interpretation (Fioretti, 2004)}

Suppose that empirical evidence is measurable and available as sets of numbers, $\left\{\mathrm{m}\left(\mathrm{A}_{1}\right), \mathrm{m}\left(\mathrm{A}_{2}\right), \cdots, \mathrm{m}(\mathrm{U})\right\}$, which represent the amounts of evidence that support subsets, $\left\{A_{1}, A_{2}, \cdots\right\}$, of a frame of discernment in $U$, respectively. Then each set of numbers, $\left\{\mathrm{m}_{1}, \mathrm{~m}_{2}, \cdots, \mathrm{m}_{\mathrm{U}}\right\}$, is called a body of evidence, where the number $\mathrm{m}$ is generally normalized to satisfy, which is expressed as Equation 28:

$$
\sum_{i} m\left(A_{i}\right)+m(U)=1, \quad \text { where } m(U)>0
$$

The numbers, $\left\{\mathrm{m}_{1}, \mathrm{~m}_{2}, \cdots\right\}$, represent amounts of empirical evidence supporting alternative possibilities $\left\{\mathrm{A}_{1}, \mathrm{~A}_{2}, \cdots\right\}$. Their meaning is clarified using an example of belief formation from the biotech industry and the ensuing discussion which is presented in (Fioretti, 2004).

\subsubsection{Combinations of Evidence}

\subsubsection{Types of Evidence (Sentz and Ferson, 2002)}

We consider four types of evidence from multiple sources that impact the choice of how information is to be combined:

- Consonant evidence

- Consistent evidence

- Arbitrary evidence

- Disjoint evidence
Evidence theory is such a framework that can handle these various evidentiary types by combining a notion of probability with the traditional conception of sets.

\subsubsection{Rules of Combinations}

Sometimes the available evidence may come from different sources. Such bodies of evidence can be aggregated using existing rules of combination (Agarwal et al., 2004). In other words, combination rules are the special types of aggregation methods for data obtained from multiple sources. Commonly used combination rules are listed below (Sentz and Ferson, 2002; Fan and Zuo, 2006a; 2006b):

- The Dempster rule of combination

- Discount $t$ combination method

- Yager's modified Dempster's rule

- Inagaki's unified combination rule

- Zhang's center combination rule

- Dubois and Prade's disjunctive consensus rule

- Fan and Zuo's improved combination rules

- Mixing or averaging

- Convolutive X-averaging

Other rules include Smets' rule, qualitative combination rule and Yen's rule (Agarwal et al., 2004).

\subsubsection{Dempster's Rule of Combination}

Although there is always a debate about the suitability of combination rules, Dempster's rule of combination is one of the most popularly used rules and could be viewed as the core of the Dempster-Shafer fusion method. The combination (called the joint $\mathrm{m}_{12}$ ) is calculated in the following manner, whicih is expressed as Equation 29:

$$
\begin{aligned}
& \left(\mathrm{m}_{1} \oplus \mathrm{m}_{2}\right)(\mathrm{A})=\mathrm{m}_{12}(\mathrm{~A}) \\
& =\frac{\sum_{\mathrm{B} \cap \mathrm{C}=\mathrm{A}} \mathrm{m}_{1}(\mathrm{~B}) \mathrm{m}_{2}(\mathrm{C})}{1-\sum_{\mathrm{B} \cap \mathrm{C}=\varnothing} \mathrm{m}_{1}(\mathrm{~B}) \mathrm{m}_{2}(\mathrm{C})}, \quad \mathrm{A} \neq \varnothing
\end{aligned}
$$

where, B and C denote propositions from each source $\left(\mathrm{m}_{1}\right.$ and $\left.\mathrm{m}_{2}\right)$.

Because of the normalization factor in the denominator, Dempster's rule is not suitable for cases where there are many inconsistencies in the available evidence, however, it is appropriate where there is some degree of consistency or sufficient agreement among the opinions of different sources (Agarwal et al., 2004). When there is little or no consistency among the evidence from different sources, the mixing or averaging rule (Sentz and Ferson, 2002) and the improved combination rule (Fan and Zuo, 2006a; 2006b) are 
available. Recent studies on combination rules fall into two categories: the methods modifying Dempster rule and the methods correcting original evidence sources (Florea et al., 2009).

\subsubsection{Advantages of Evidence Theory}

Hegarat-Mascle et al. (1997); Agarwal et al. (2004) and Nikolaidis and Haftka (2001):

- Evidence theory can model both reducible and irreducible uncertainty when the amount of information available is small. For instance, one can express his ignorance of the likelihood of a certain event being extremely small by assigning a large plausibility and a low belief to it, which appears as a more flexible and general approach than the Bayesian one

- Evidence theory provides a measure of the uncertainty in estimating of risk. The wider the gap between plausibility and belief in the previous example, the greater the uncertainty about the estimated risk of the event

- Evidence theory considers not only single or individual classes, but also unions of classes

\subsubsection{Disadvantages of Evidence Theory}

In the present literature, the criticism of the Dempster-Shafer theory of evidence is crucially based on the following points (Kozine and Filimonov, 2000):

- Failure to produce rational results in the case of inconsistent combined pieces of information according to Dempster's rule of combination

- Inability to combine opinions of different people with overlapping experiences, especially in safety analysis application (Wu et al., 1990)

- Be formally incoherent in safety assessment similar to the theory of probability

To implement the Dempster-Shafer and possibility theories into risk and reliability analyses, Kozine and Filimonov (2000) also encountered some difficulties that could not be solved in the frameworks of these theories. They summarize the main drawbacks as follows:

- Combination of homogeneous bodies of evidence

- Combination of inconsistent pieces of information

- Judgments admitted in elicitation

- Dependence of imprecision on the amount of information

This indicates, in a final personal opinion, Dempster's rule of combination can produce formally incoherent inferences.

\section{COMPARISON OF POSSIBILITY THEORY AND PROBABILITY THEORY}

When Zadeh (1978) proposed the possibility theory, he pointed out that additional insight into the distinction between probability and possibility may be gained by comparing the concept of a possibility measure with the familiar concept of a probability measure. Since then, the debate between possibility theory and probability theory has been on-going. We believe that the focus should be on the specific practical surroundings a certain method is available to, rather than which is better than the other. After all, each concept is useful in its own domain and blending is normal in several situations, as Kaufmann (1983) said.

There are rich studies that compare probability theory with possibility theory, or with fuzzy sets theory and evidence theory (Liu et al., 2009; 2012; Greene et al., 2011; Klir and Yuan, 1995; Misra and Soman, 1995; Utkin et al., 1995; Cai, 1996; Cayrac et al., 1996; Ferson and Ginzburg, 1996; Huang, 1996; Utkin and Gurov, 1996). Discussions mainly focus on the aspect of axiom, from which we conclude that a principal difference between these theories is that the probability theory's additivity axiom (about the probability of the disjointed events uniting) is replaced with less restrictive axioms. Some comparisons consider the notion of consistency between possibilities and probabilities (Delgado and Moral, 1987; Dubois and Prade, 1983). Some focus on the transformations from probabilities and possibilities to evidence theory (Klir and Parviz, 1992). Their comparisons are quantitative in terms of both efficiency and expressiveness, but cannot give the exact relationship between probabilities and possibilities. Thus Drakopoulos (1995) studies extensions of the universal sets, mapping among probabilities, possibilities and fuzzy sets in order to specify their important relationships. Other comparisons are from the angle of design rather than theory. Chen et al. (1999) compare probabilistic and fuzzy set models for design against uncertainty when there is limited information about the statistics of the uncertainty or modeling error. They conclude that if there is sufficient information for building accurate probabilistic models of uncertainties, probabilistic methods are better than fuzzy set methods. Soundappan et al. (2004) compare evidence theory and Bayesian theory for uncertainty modeling and decisionmaking under uncertainty.

Although each measure or concept of the different theories is useful in its own domain and has its own definition and application, consideration and blending is normal in several situations (Kaufmann, 1983). Comparisons are necessary in order to evaluate and characterize those measures. 


\subsection{Similarities}

Both possibility theory and probability theory are used for uncertainty analysis and quantification. They are both subsumed under the mathematical theory of evidence (or DST).

Possibility theory has been based on distributions in the same way as probability theory has been. Fuzzy variables are associated with possibility distributions in the same way as random variables are associated with probability distributions.

Possibility measures, analogous to probability measures, constitute tools for representing and quantifying uncertainty. Probability and possibility measures, i.e., fuzzy measures, are all nonnegative and monotonic (Klir and Yuan, 1995; Nikolaidis and Haftka, 2001).

\subsection{Differences}

Generally, although probability and possibility measures are both tools for representing uncertainty and their adopted theories and methods are related to each other, the two concepts are essentially different. Unlike probability, possibility is not subject to repeated experiments and hence does not refer to statistic properties. Moreover, in terms of observating various circumstances, a probability measure assesses odds of occurrence, whereas possibility assesses degree of ease. Hence, unlike the classical probability theory which is best suited to aleatory uncertainty, possibility theory is usually used to quantify only epistemic uncertainty. With a view to the information involved, probability is a quantitative ratio scale of uncertainty while possibility is a quasi-qualitative ordinal scale. Thus there is an opinion (Dubois et al., 1993) that there is no more information included in a possibility distribution than in a probability distribution. We analyze and discuss both in the following.

Let $U$ be the universal set and $A$ be a set of crisp subsets of $\mathrm{U}$; comparisons of some basic formulae in possibility theory and probability theory are given in Table 3. To some extent, probability is a ratio scale of uncertainty while possibility can be considered as an ordinal scale. Consequently, the numerical values for probability have their own meaning. On the other hand, the essential information from a possibility distribution is the order of elements for possibilities; numerical values considered are just an expedient way of specifying the order (Dubois and Prade, 1983; Dubois et al., 1993).

\subsubsection{Axiomatic Differences}

A major difference between probability and possibility can be found in axioms about the union of events (disjoint or overlapping), that probability is additive whereas possibility is sub-additive (Nikolaidis and Haftka, 2001).
Table 3. Comparison of possibility theory and probability theory

\begin{tabular}{|c|c|}
\hline Probability & possibility \\
\hline $\begin{array}{l}\text { Distribution } \prod \text { measure } \pi \\
\sup _{\mathrm{u} \in \mathrm{U}} \pi(\mathrm{u})=1\end{array}$ & $\begin{array}{l}\text { Distribution P, Probability } p \\
\sum_{u \in U} p(u)=1\end{array}$ \\
\hline$\forall(\mathrm{A}, \mathrm{B}) \in \wp(\mathrm{U})$ & $\forall(\mathrm{A}, \mathrm{B}) \in \wp(\mathrm{U})$ \\
\hline $\mathrm{P}_{\mathrm{oss}}(\mathrm{A})=\pi(\mathrm{A})=\sup _{\mathrm{u} \in \mathrm{A}} \Pi(\mathrm{u})$ & $\mathrm{P}_{\mathrm{ro}}(\mathrm{A})=\mathrm{p}(\mathrm{A})=\sum_{\mathrm{u} \in \mathrm{A}} \mathrm{P}(\mathrm{u})$ \\
\hline$\pi(\mathrm{A} \cup \mathrm{B}), \max (\pi(\mathrm{A}), \pi(\mathrm{B}))$ & $\begin{array}{l}\mathrm{p}(\mathrm{A} \cup \mathrm{B})=\mathrm{p}(\mathrm{A})+\mathrm{p}(\mathrm{B}) \\
\text { when } \mathrm{A}, \mathrm{B} \text { is disjoint }\end{array}$ \\
\hline $\mathrm{N}_{\mathrm{ec}}(\mathrm{A} \cap \mathrm{B})=\min \left(\mathrm{N}_{\mathrm{ec}}(\mathrm{A}), \mathrm{N}_{\mathrm{ec}}(\mathrm{B})\right)$ & $\mathrm{p}(\mathrm{A} \cap \mathrm{B}) \leq \mathrm{p}(\mathrm{A}) \overline{\mathrm{p}}(\mathrm{B})$ \\
\hline$\left\{\begin{array}{l}\max (\pi(\mathrm{A}), \pi(\overline{\mathrm{A}}))=1 \\
\pi(\mathrm{A})+\pi(\overline{\mathrm{A}}) \geq 1 \\
\mathrm{~N}_{\mathrm{ec}}(\mathrm{A})+\mathrm{N}_{\mathrm{ec}}(\overline{\mathrm{A}}) \leq 1\end{array}\right.$ & $\mathrm{p}(\mathrm{A})+\mathrm{p}(\overline{\mathrm{A}})=1$ \\
\hline$\forall \mathrm{u} \in \mathrm{U}, \quad \Pi(\mathrm{x})=1$ & $\forall \mathrm{u} \in \mathrm{U}, \quad \mathrm{P}(\mathrm{x})=\frac{1}{|\mathrm{U}|}$ \\
\hline
\end{tabular}

In other words, the possibility of the union of a finite number of events (disjoint or not) is equal to the maximum of the possibilities of the individual events, whereas the probability of a union of disjoint events is equal to the sum of their probabilities. As well, the probability of a union of an event and its negation must add up to 1 (Chen et al., 1999); Table 2. The additive rule is the basic feature of probability theory (including classical and Bayesian probability theory).

The difference between possibilities and probabilities can also be seen in the context of fuzzy measure. Sugeno introduced fuzzy measure as a generalization of real measures. Fuzzy measure is a continuous or semicontinuous function from a class of crisp sets of a power set to the interval $[0,1]$. When the universal set is finite, probability and possibility measures are special cases of the fuzzy measure (Chen et al., 1999). Table 4 compares these measures in terms of their axioms (Klir, 2000; Klir and Folger, 1988; Klir and Yuan, 1995). Let U be the universal set and $A$ be a set of crisp subsets of $U$.

So the consistency of probability measure, possibility measure and necessity measure is expressed below $\forall \mathrm{A} \in \wp(\mathrm{U})$ Equation 30:

$\mathrm{N}_{\mathrm{ec}}(\mathrm{A}) \leq \mathrm{P}(\mathrm{A}) \leq \mathrm{P}_{\mathrm{oss}}(\mathrm{A})$

Note that, of course, the above requirement of the same event is based on intuition and cannot be proven mathematically (Chen et al., 1999).

\subsubsection{Calculi Differences}

Because of the axiomatic difference, probability and possibility calculi are fundamentally different and one cannot simulate possibility calculus using probabilistic models (Nikolaidis et al., 2004). 
Table 4. Comparison of possibility measure and probability measure

\begin{tabular}{|c|c|c|c|}
\hline & Fuzzy measure $g(*)$ & Probability measure $\mathrm{p}\left({ }^{*}\right)$ & Possibility measure $\pi(*)$ \\
\hline Boundary conditions & $\mathrm{g}(\varnothing)=1, \mathrm{~g}(\mathrm{U})=0$ & $\mathrm{p}(\mathrm{U})=1$ & $\pi(\varnothing)=1, \pi(\mathrm{U})=0$ \\
\hline \multirow[t]{3}{*}{ Monotonicity } & $\begin{array}{l}\text { For all, } \mathrm{A}, \mathrm{B} \in \mathrm{U} \text {, } \\
\text { if } \mathrm{A} \subseteq \mathrm{B} \text { then } \mathrm{g}(\mathrm{A}) \leq \mathrm{g}(\mathrm{B}) \\
\text { (continuity from below) }\end{array}$ & $\mathrm{P}(\mathrm{A}) \geq 0$ when $\mathrm{A} \in \mathrm{U}$ & $\begin{array}{l}\text { For all, } A, B \in U \text { if } A \subseteq B \text {, } \\
\text { then } \pi(A) \leq(B) \pi\end{array}$ \\
\hline & For all $A_{1} \subseteq A_{2} \subseteq \cdots$ if $\bigcup_{i=1}^{\infty} A_{i} \in U$ & $\begin{array}{l}\forall A_{i}, i \in I \\
\text { where } A_{i} \text { is disjoint, } \\
p\left(\bigcup_{i=1}^{I} A_{i}\right)=\sum_{i \in I} p\left(A_{i}\right)\end{array}$ & $\begin{array}{l}\forall A_{i}, i \in I \\
\text { where } A_{i} \text { is disjoint, } \\
\pi\left(\bigcup_{i=1}^{I} A_{i}\right)=\max _{i \in I}\left(\pi\left(A_{i}\right)\right)\end{array}$ \\
\hline & Then $\lim _{i \rightarrow \infty} g\left(A_{i}\right)=g\left(\bigcup_{i=1}^{\infty} A_{i}\right)$ & & \\
\hline \multirow[t]{3}{*}{ Continuity } & (continuity from above) & & \\
\hline & For all $A_{1} \supseteq A_{2} \supseteq \cdots$ if $\bigcap_{i=1}^{\infty} A_{i} \in U$ & & \\
\hline & Then $\lim _{i \rightarrow \infty} g\left(A_{i}\right)=g\left(\bigcap_{i=1}^{\infty} A_{i}\right)$ & & \\
\hline
\end{tabular}

Nikolaidis et al. (2004) and Nikolaidis and Haftka (2001) compare probability and possibility by means of uncertainty modeling. They summarize the main differences as follows.

Methods of modeling an uncertain quantit: probability theory models an uncertain quantity by using its probability distribution function, $\mathrm{F}(\mathrm{x})$ and its probability density function, $f(x)$, the latter being a derivative of the former. Correspondingly, possibility theory uses the possibility distribution function, $\pi(\mathrm{u})$, which is the function form and numerical expression of the possibility distribution, $\Pi(\mathrm{u})$; in respect that possibility is both a measure and a function. Some differences between the probability density and possibility distribution of a continuous variable are summarized below:

- The area below the probability density function is one whereas the area below the possibility distribution function has no such meaning and can be any value

- The probability of a continuous variable which takes value in an infinitesimal interval is usually zero, whereas the possibility of the same case is usually greater than zero

- The maximum value of the probability density function can be greater than one and the value of the possibility distribution is not greater than one

The notion of independence of events; in probability, we say two events are independent if and only if Equation 31 is hold:

$\mathrm{p}(\mathrm{A} \cap \mathrm{B})=\mathrm{p}(\mathrm{A}) \cdot \mathrm{p}(\mathrm{B})$

In the case of possibility theory, there does not exist an exact definition of independence. In order to express the fact that two events are not interdependent, we say they are non-interactive when Equation 7 holds with the equality sign, i.e., Equation 32:

$\pi(\mathrm{A} \cap \mathrm{B})=\pi(\mathrm{A}) \wedge \pi(\mathrm{B})$

This indicates that independence is a stronger condition than non-interaction. In other words, if we decrease the possibility of the least possible event, we cannot compensate for the entailed reduction in the possibility by increasing the possibility of the other events (Nikolaidis et al., 2004).

\subsubsection{Realization Differences}

The above comparisons affirm two statements. In the epistemological position, probability theory, which is based on classical set theory, abandons the law of causation; whereas possibility theory, which is based on fuzzy set theory, abandons the law of balance. In the degree of information required, probability theory is best suited to aleatory uncertainty when there is sufficient input data, whereas possibility theory is usually used to quantify only epistemic uncertainty even if insufficient information is available.

As a result, probability theory is popular in statistic technologies, data analysis and communication systems. On the other hands, applications of possibility theory can be found in industrial process control, pattern recognition and group decision-making.

\subsection{Possibility-Probability Principles}

Consistency

If a probability and a possibility are assigned to the same event, then one expects that the possibility of that 
event should be consistent with its probability, i.e., the possibility should be equal to or greater than the probability of the event, seen in inequality Equation 30 . This is a logical interpretation of possibility-probability consistency principles.

Zadeh (1978) has used a famous example of eating eggs to illustrate the difference between probability and possibility. His observation is that Equation 33 and 34:

high probability $\underset{\mathrm{No}}{\stackrel{\mathrm{Yes}}{\rightleftarrows}}$ hign possibility

low probability $\underset{\text { Yes }}{\stackrel{\text { No }}{\rightleftarrows}}$ low possibility

Such connection is named as the possibility/probability consistency principle. If a variable $\mathrm{X}$ can take the values $\mathrm{u}_{1}, \mathrm{u}_{2}, \ldots, \mathrm{u}_{\mathrm{n}}$ with respective possibilities $\Pi=\left(\pi_{1}, \ldots, \pi_{n}\right)$ and probabilities $P=\left(p_{1}, \ldots\right.$, $\mathrm{p}_{\mathrm{n}}$ ), then the degree of consistency of the probability distribution $\mathrm{P}$ with the possibility distribution $\Pi$ is expressed by by Equation 35:

$\gamma=\pi_{1} p_{1}+\cdots+\pi_{n} p_{n}$

Note that, of course, the principle is not a precise law or an intrinsic relationship rather it is an approximate formalization of a heuristic observation; this means that decreasing the possibility of an event leads to decreasing its probability-but the obverse is not true.

Zadeh's original motivation for the possibility/probability consistency principle is to provide a basis for computing of possibility distribution from the probability distribution of X (Zadeh, 1978). Such computation is important in decision-making under uncertainty and in the evidence and belief theories.

\section{COMPATIBILITY OF EVIDENCE THEORY WITH POSSIBILITY THEORY AND PROBABILITY THEORY}

As expatiated above attempts at quantifying uncertainty using possibilities, probabilities and fuzzy sets share some common properties but also display important differences (Borotschnig et al., 1999). We may induce a compatibility capacity of evidence theory, with possibility theory and probability theory. Briefly we can say that the classical probability theory and the possibility theory are subsets of the evidence theory (Mourelatos and Zhou, 2004). In other words, both of them are complementary or alternative theories, neither of which is a generalization of the other (Klir and Folger, 1988); Fig. 5.

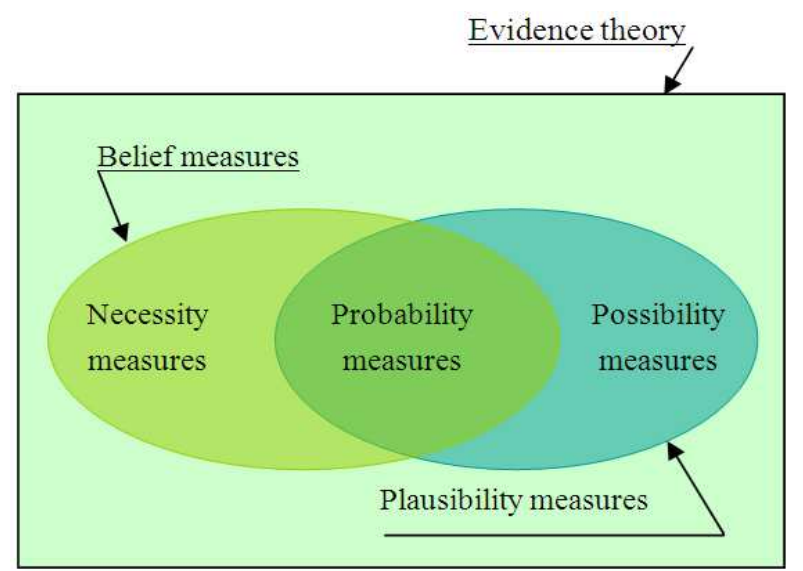

Fig. 5. A pictorial description of uncertainty classification based on fuzzy measures

\subsection{Connection of Possibility Theory with Evidence Theory}

Possibility theory may be viewed as a special branch of fuzzy measure theory (Klir and Folger, 1988). Fuzzy measure theory is based on two dual fuzzy measures, $\mathrm{P}_{\text {oss }}$ and $\mathrm{N}_{\mathrm{ec}}$, which are connected with the corresponding two measures, $\mathrm{B}_{\mathrm{el}}$ and $\mathrm{P}_{1}$ from evidence theory. This is expatiated below.

Function $\mathrm{m}$, defined in Equation 17, i.e., basic probability assignment in evidence theory, is an alternative representation of the possibility measure. As determined by (Klir, 1995), when all focal elements in evidence theory are nested or consonant, provided that $\mathrm{m}$ (A) $>0$, we obtain a special plausibility measure which is expressed in Equation 36:

$$
\pi: U \rightarrow[0,1]
$$

Via the Equation 37 (Klir, 2000):

$$
\mathrm{P}_{\text {oss }}(\mathrm{A})=\sup _{\mathrm{u} \in \mathrm{A}} \pi(\mathrm{u})
$$

which derives from Equation 17 and 20 and resembles Equation 4. This special plausibility measure is actually called a possibility measure in possibility theory. Moreover, the corresponding special belief measure, the alternative to the two dual measures in evidence theory, is called a necessity measure. It is defined in Equation 18 as $\mathrm{N}_{\mathrm{ec}}(\mathrm{A})=1-\mathrm{P}_{\mathrm{oss}}(\overline{\mathrm{A}})$. In short, when all the focal elements are nested or consonant, the $\mathrm{P}_{1}$ and $\mathrm{B}_{\mathrm{el}}$ in 
evidence theory are equal to the $P_{o s s}$ and $N_{e c}$ in possibility theory. Here, the nested structure implies that $\pi_{1}^{3} \pi_{\mathrm{i}+1}$, for all $\mathrm{i}=1,2, \mathrm{~L}, \mathrm{n}-1$, where $\pi=\left(\pi_{1}, \pi_{2}, \mathrm{~L}, \pi_{\mathrm{n}}\right)$. When $\mathrm{m}(\mathrm{A}) \neq 0$, it is induced (Klir, 1995) that Equation 38 and 39 :

$$
\begin{aligned}
& \pi_{\mathrm{i}}=\sum_{\mathrm{l}=\mathrm{i}}^{\mathrm{n}} \mathrm{m}_{1} \\
& \mathrm{~m}_{\mathrm{i}}=\pi_{\mathrm{i}}-\pi_{\mathrm{i}+1}
\end{aligned}
$$

The pair, $\langle\mathbb{F}, \mathrm{m}\rangle$, where $\mathbb{F}$ denotes the set of all focal elements induced by $\mathrm{m}$, is called a body of evidence. Thus possibility theory deals with special bodies of evidence, where $\mathbb{F}$ is a nested family of subsets of $U$ in each body of evidence. In addition to the well-known fuzzy-set interpretation, possibility can also be interpreted in the framework of evidence theory, as a subset of evidence theory (Mourelatos and Zhou, 2004).

\subsection{Evidence Theory as an Extension of Probability Theory}

Evidence theory uses two large classes of measures of uncertainty, $\mathrm{B}_{\mathrm{el}}$ and $\mathrm{P}_{1}$. In comparison, probability theory uses just one measure, the probability of an event, i.e., $\mathrm{P}_{\text {ro }}$.

Assuming all focal elements in evidence theory are singletons, consider an imprecise set of probabilities expressed by the interval $\left[\mathrm{B}_{\mathrm{el}}(\mathrm{A}), \mathrm{P}_{1}(\mathrm{~A})\right]$. For all $A \in \wp(U)$, there is then $B_{e l}(A)=P_{1}(A)$. This gives us a classical probability measure, $\mathrm{P}_{\text {ro, }}$, which is determined by Equation 40:

$$
\mathrm{p}: \mathrm{U} \rightarrow[0,1]
$$

Via the Equation 41:

$$
\mathrm{P}_{\mathrm{ro}}(\mathrm{A})=\sum_{\mathrm{u} \in \mathrm{A}} \mathrm{p}(\mathrm{u})
$$

where, $\mathrm{p}(\mathrm{u})$ is the classical probability distribution function (PDF). Compared with Equation 19 and 20 p(u) $=\mathrm{m}(\{\mathrm{u}\})$ for all $\mathrm{u} \in \mathrm{U}$.

Since evidence theory deals with imprecise probabilities (Walley, 1991), when handling a mixture of input parameters from incomplete data, the range of each input can be described as falling within a specific interval, $\left[\mathrm{B}_{\mathrm{el}}(\mathrm{A}), \mathrm{P}_{1}(\mathrm{~A})\right]$. If the plausibility measure and the belief measure are equal, it follows logically from Equation 40 and 41 that classical probability theory is a special case of evidence theory. In other words, when the ignorance of uncertainty reaches the value zero, evidence theory can be considered as a generalization of probability theory; this is because its measure falls below those associated with probability theory.

Strictly speaking, classical probability theory is a subset of possibility theory, which in turn (Fig. 5) is a subset of evidence theory. The transformation between probability measure and possibility measure is discussed in the next sub-section.

\subsection{Probability-Possibility Transformation}

In order to comprehend the relationship between probability theory and possibility theory and to realize how compatible of evidence theory is with these two theories, many researchers have studied transformations between probability and possibility; this provides a theoretical background for practical problems of system modeling, decision making and analysis of data and expert systems. Most researchers examined the principles to be satisfied for transformation in a heuristic way (Liu et al., 2009; Dubois and Prade, 1983; Dubois et al., 1993; Oberkampf et al., 2000; Yamada, 2001; Zadeh, 1978) asserting that their propositions are the only specific ones to satisfy until Yamada (2001) devised three new transformation methods based on evidence theory and declared these three transformations to be the only ones that satisfy the principles.

Let's review the already-existing transformations (Yamada, 2001; Zadeh, 1978) now.

\subsubsection{Zadeh's Consistency Principle}

Zadeh illustrated the relationship between probability and possibility (See inequality (30)), proposed the consistency principle expressed by Equation 33 and 34 and defined the degree of consistency signified as Equation 35, i.e. $\gamma=\pi_{1} p_{1}+\cdots+\pi_{n} p_{n}$. From the basic properties of the possibility and necessity measures (Klir, 2000) we know that maximizing the degree of consistency brings a strong restrictive condition as below Equation 42 and 43:

$$
\begin{aligned}
& \mathrm{P}_{\mathrm{oss}}(\mathrm{A})<1 \Rightarrow \mathrm{N}_{\mathrm{ec}}(\mathrm{A})=0 \\
& \mathrm{~N}_{\mathrm{ec}}(\mathrm{A})>0 \Rightarrow \mathrm{P}_{\mathrm{oss}}(\mathrm{A})=1
\end{aligned}
$$

which demand that Equation 30, i.e., $\mathrm{N}_{\mathrm{ec}}(\mathrm{A}) \leq \mathrm{P}(\mathrm{A}) \leq$ $\mathrm{P}_{\text {oss }}(\mathrm{A})$, should be satisfied in general.

\subsubsection{Dubois and Prade's Transformation from a Histogram}

Dubois and Prade asserted that two principles must be satisfied first, one is Equation 13, i.e., 
$\mathrm{P}_{\text {oss }}(\mathrm{A}) \geq \mathrm{N}_{\mathrm{ec}}(\mathrm{A})$ and the other is a revision of Equation 33 which is expressed as Equation 44:

$$
\mathrm{p}\left(\mathrm{u}_{\mathrm{i}}\right)>\mathrm{p}\left(\mathrm{u}_{\mathrm{j}}\right) \Leftrightarrow \pi\left(\mathrm{u}_{\mathrm{i}}\right)>\pi\left(\mathrm{u}_{\mathrm{j}}\right)
$$

These two principles are also called probability/possibility consistency and preference preservation Dubois et al. (1993) and Dubois and Prade (1983) proposed the transformation between probability and possibility from a histogram as the following equation, when the probability of $\mu_{\mathrm{i}}$ is an degressive sequence, $p\left(u_{1}\right) \geq p(u) \geq \ldots \geq p\left(u_{n}\right)$, then:

$$
\begin{aligned}
& \pi_{\mathrm{x}}\left(\mathrm{u}_{\mathrm{i}}\right)=\Pi\left(\left\{\mathrm{u}_{\mathrm{i}}\right\}\right) \\
& =\sum_{\mathrm{j}=\mathrm{i}}^{\mathrm{n}} \min \left(\mathrm{p}\left(\mathrm{u}_{\mathrm{i}}\right), \mathrm{p}\left(\mathrm{u}_{\mathrm{j}}\right)\right) \\
& =\mathrm{i} \cdot \mathrm{p}\left(\mathrm{u}_{\mathrm{i}}\right)+\sum_{\mathrm{j}=\mathrm{i}+1}^{\mathrm{n}} \mathrm{p}\left(\mathrm{u}_{\mathrm{j}}\right)
\end{aligned}
$$

where, $\sum_{j=i+1}^{n} p\left(u_{j}\right)=0$ and $u_{i}$ is an element of variable $X$ taking value in a universe discourse, U. Of course, Equation 45 can be transformed in the converse direction. Generally, a possibility distribution can be obtained by normalizing a histogram as Equation 46:

$$
\pi_{\mathrm{X}}\left(\mathrm{u}_{\mathrm{i}}\right)=\frac{\mathrm{p}\left(\mathrm{u}_{\mathrm{i}}\right)}{\max _{\mathrm{i}=1}^{\mathrm{n}} \mathrm{p}\left(\mathrm{u}_{\mathrm{i}}\right)}
$$

Although both consistency and preference principles are satisfied, this transformation shows no guarantee to be just the only available one (Yamada, 2001).

\subsubsection{Transformation Based on Maximal Specificity}

From the comparison of possibility and probability as certain uncertainty quantifications as stated above, aware of Zadeh's investigation of the relationship between possibility and information and having seen the unique connection between uncertainty and information pointed out by Klir (1995), we now know there is less information covered by a possibility distribution than by a probability distribution. This suggests a principle that possibility distributions generated from probability distributions should include the fewest possible number of fuzzy sets in order to remain maximally specific (Cai et al., 1993).

Given a possibility and probability distribution, the transformation under the maximally specific principle, satisfying the consistency principle (Equation 13 and 44) can be expressed as Equation 47 and 48 :

$$
\begin{aligned}
& \pi_{\mathrm{x}}\left(\mathrm{u}_{\mathrm{i}}\right) \geq \sum_{\mathrm{j}=\mathrm{i}}^{\mathrm{n}} \mathrm{p}\left(\mathrm{u}_{\mathrm{j}}\right), \text { when } \pi\left(\mathrm{u}_{1}\right) \geq \pi\left(\mathrm{u}_{2}\right) \geq \cdots \geq \pi\left(\mathrm{u}_{\mathrm{n}}\right) \\
& \pi_{\mathrm{x}}\left(\mathrm{u}_{\mathrm{i}}\right)=\sum_{\mathrm{j}=\mathrm{i}}^{\mathrm{n}} \mathrm{p}\left(\mathrm{u}_{\mathrm{j}}\right) \text {, when } \mathrm{p}\left(\mathrm{u}_{1}\right) \geq \mathrm{p}\left(\mathrm{u}_{2}\right) \geq \cdots \geq \mathrm{p}\left(\mathrm{u}_{\mathrm{n}}\right)
\end{aligned}
$$

Yamada proved that the above condition as Equation 48 is not adequate for the consistency principle.

\subsubsection{Klir's Transformation Based on Uncertainty Invariance}

In accordance with the principle of uncertainty and information invariance (Klir, 1995; Klir and Folger, 1988) which claims that the amount of uncertainty should be preserved and the degree of belief should be converted by an appropriate scale when information flows through different uncertainty models in different theories, Geer and Klir (1988) and Klir and Parviz (1992) proposed that transformation be based on the principle of uncertainty invariance, also called the principle of information preservation or information invariance. These principles defining the relationship between probability and possibility, were also derived by Jumarie (1994) and developed by Wonneberger (1994).

Geer and Klir first defined two kinds of possibilistic uncertainties on the body of evidence called nonspecificity $\mathrm{N}$ and strife (or discord), $\mathrm{S}$, for each ordered degressive possibility distribution on a set with $n$ elements. These were expressed as Equation 49 and 50:

$$
\begin{aligned}
& \mathrm{N}(\pi)=\sum_{\mathrm{i}=2}^{\mathrm{n}}\left(\pi_{\mathrm{i}}-\pi_{\mathrm{i}+1}\right) \log _{2} \mathrm{i} \\
& \mathrm{S}(\pi)=\sum_{\mathrm{i}=2}^{\mathrm{n}}\left(\pi_{\mathrm{i}}-\pi_{\mathrm{i}+1}\right) \frac{\mathrm{i}}{\sum_{\mathrm{j}=1}^{\mathrm{i}} \pi_{\mathrm{j}}}
\end{aligned}
$$

This transformation has been investigated under such assumption that the total possibilistic uncertainty is measured by the sum of $\mathrm{N}$ and $\mathrm{S}$ and the total uncertainty must be preserved in the transformation process.

Then Geer and Klir give the log-interval scale transformation satisfying the principle in both directions, if $\mathrm{p}\left(\mathrm{u}_{1}\right) \geq \ldots \geq \mathrm{p}\left(\mathrm{u}_{\mathrm{n}}\right), \pi\left(\mathrm{u}_{1}\right) \geq \pi\left(\mathrm{u}_{2}\right) \geq \ldots \geq \pi\left(\mathrm{u}_{\mathrm{n}}\right)$, then $\pi_{\mathrm{X}}\left(\mathrm{u}_{\mathrm{i}}\right)$ can be expressed as Equation 51:

$\pi_{\mathrm{x}}\left(\mathrm{u}_{\mathrm{i}}\right),\left(\frac{\mathrm{p}\left(\mathrm{u}_{\mathrm{i}}\right)}{\mathrm{p}\left(\mathrm{u}_{1}\right)}\right)^{\alpha}, \quad \alpha \in(0,1)$ 
Hong-Zhong Huang et al. / American Journal of Engineering and Applied Sciences 6 (1): 95-136, 2013

Table 5. Probability-possibility transformation based on evidence theory (Yamada, 2001)

\begin{tabular}{lll}
\hline Possibility types & Given principles & Transformation expressions \\
\hline $\mathrm{T}_{1}$ : Ordinal scale & Probabilistic order preservation principle & $\mathbb{F}_{\mathrm{k}}^{\pi}=\bigcup_{\mathrm{h}=1}^{\mathrm{k}} \mathrm{U}_{\mathrm{h}}, \mathrm{k}=1, \cdots, \mathrm{K}$ \\
$\mathrm{T}_{2}$ : Ratio scale (1) & Consistency principle and probabilistic order preservation principle & $\pi\left(\mathrm{u}_{\mathrm{i}}\right)=\left\{\begin{array}{l}\sum_{\mathrm{k}=\mathrm{q}_{\mathrm{j}}}^{\mathrm{n}} \mathrm{p}\left(\mathrm{u}_{\mathrm{k}}\right), \mathrm{q}_{\mathrm{j}} \leq \mathrm{i} \leq \mathrm{r}_{\mathrm{j}}(\mathrm{j}=1, \cdots, \mathrm{m}) \\
\sum_{\mathrm{k}=\mathrm{i}}^{\mathrm{n}} \mathrm{p}\left(\mathrm{u}_{\mathrm{k}}\right), \text { otherwise }\end{array}\right.$ \\
$\mathrm{T}_{3}$ : Ratio scale (2) & Equidistribution principle & $\mathrm{p}\left(\mathrm{u}_{\mathrm{i}}\right)=\mathrm{m}_{\mathrm{p}}\left(\left\{\mathrm{u}_{\mathrm{i}}\right\}\right)=\sum_{\mathrm{h}=\mathrm{k}}^{\mathrm{K}} \mathrm{m}_{\pi}\left(\mathbb{F}_{\mathrm{h}}^{\pi}\right) /\left|\mathbb{F}_{\mathrm{h}}^{\pi}\right|$, \\
& & $\forall \mathrm{u}_{\mathrm{i}} \in \mathrm{G}_{\mathrm{k}}^{\pi}=\mathbb{F}_{\mathrm{k}}^{\pi}-\mathbb{F}_{\mathrm{k}-1}^{\pi}$ \\
\hline
\end{tabular}

where, coefficient $\alpha$ is a constant satisfying a unique equation $g(\alpha)=0$ (Klir, 1995) and $\alpha \in(0,1)$ implies that the consistency condition, $\mathrm{N}_{\mathrm{ec}}(\mathrm{A}) \leq \mathrm{P}(\mathrm{A}) \leq \mathrm{P}_{\text {oss }}$, is also satisfied in general.

Actually, the flexibility of such transformations has not been fully investigated since first explored (Klir and Parviz, 1992). The uncertainty-invariant transformations are also questioned with regard to several aspects. Even Klir himself has realized that the uncertainty-invariant transformations are not unique to ordinal scales, although that is a disadvantage. From another point of view, it is an advantage that additional requirements can be imposed on the transformation because of the lack of uniqueness. Moreover, the principle of information preservation, as proposed by Klir, is incompatible with the basis of maximal specificity. In addition, we are not sure whether or not a function relationship such as Equation 51 is universal between such a possibility-probability transformation.

\subsubsection{Yamada's Transformation Based on Evidence Theory}

Now that evidence theory, which proved to be an amalgamation of probability theory and possibility theory, is particularly useful for representing and combining uncertain information when a single, precise, uncertainty model is unavailable, transformations between probability and possibility will also point to base of evidence theory. Yamada (2001) devises three new transformation methods based on evidence theory when possibility is considered as three cases respectively and finds these transformations generate the same ordinal structure of possibility, moreover can be the only ones satisfying the transformation principles.

In Yamada's transformations, let $(\mathrm{F}, \mathrm{m})$ be the body of evidence, then $\mathrm{E}_{\mathrm{p}}=\left(\mathrm{F}^{\mathrm{p}}, \mathrm{m}_{\mathrm{p}}\right)$ and $\mathrm{E}_{\pi}=\left(\mathrm{F}^{\pi}, \mathrm{m}_{\pi}\right)$ are bodies of evidence to define probability and possibility distribution, respectively. So the transformation between $\mathrm{p}\left(\mathrm{u}_{\mathrm{i}}\right)$ and $\pi\left(\mathrm{u}_{\mathrm{i}}\right)$ can be substituted for the transformation $\mathrm{E}_{\mathrm{p}}$ and $\mathrm{E}_{\pi}$, where $\mathrm{m}_{\mathrm{p}}=\left(\left\{\mathrm{u}_{\mathrm{i}}\right\}\right)=\mathrm{p}\left(\mathrm{u}_{\mathrm{i}}\right)$ and the focal

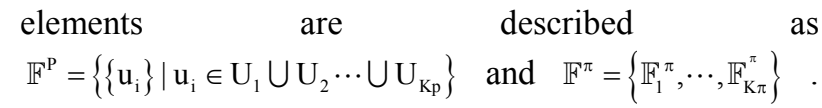

The other symbols (e.g., $U_{k}$ and $K$ ) can be seen in (Yamada, 2001).

The transformations based on evidence theory, more exactly bodies of evidence are as follows in Table 5.

Here the three cases $T_{1}, T_{2}$ and $T_{3}$ are all transformations from a given probability distribution into a possibility one. But the case $\mathrm{T}_{2}$ is not the inverse transformation whereas the third case $T_{3}$ is one applicable in both directions. So, when possibility is regarded as a ratio scale, considering the given principles (consistency principle, probabilistic order preservation principle and equidistribution principle) simultaneously, the transformation $T_{3}$ is more valid than $T_{2}$.

\section{POSSIBILITY AND EVIDENCE-BASED RELIABILITY ANALYSIS AND DESIGN OPTIMIZATION}

Before the acceptance and adoption of possibility theory and evidence theory in engineering areas, probability theory had shown its effectiveness and had gained popularity in many applications such as modeling and quantifying uncertainty in engineering systems or structural designs instead of simply assigning safety factors.

Possibility theory and evidence theory offer alternatives to traditional probabilistic theory, ones that provide for the mathematical representations of uncertainty for complex and sophisticated systems. They can also be used when there is insufficient information about random variations because they make possible a combinatorial interval analysis. Both possibility theory and evidence theory have been used recently in reliability analysis and optimization. Since Zadeh (1978) published his famous paper on possibility theory, possibility theory has received more and more attention from researchers in an increasing range of scientific areas, including reliability analysis and uncertainty 
management, so too has evidence theory.

Here we mainly discuss applying possibility theory and evidence theory to engineering design and analysis situations. As well, it is epistemic uncertainty that this study focuses on primarily.

\subsection{General Topics of Applications}

Possibility theory has been applied in many areas. It is usually used to quantify epistemic uncertainty if there is no conflicting evidence among experts, unlike classical probability theory, which is best suited to aleatory uncertainty (Bae et al., 2004a). In the area of reliability engineering, reliability estimation and design are investigated by (Mourelatos and Zhou, 2004), Kozine and Filimonov (2000); Moller et al. (1999; 2004); Huang (1995; 1996); Huang et al. (2006a; 2006b; 2010); Huang (2012); Li et al. (2012); Pang et al. (2012); Wang et al. (2011; 2012) and Xiao et al. (2012). Modeling of reliability using a new data fusion rule is proposed by Delmotte and Borne (1998); Sun et al. (2008) and Yang et al. (2011a); Possibility-based design optimization is studied and developed by (Mourelatos and Zhou, 2004; Youn, 2005; Youn and Choi, 2004a; 2004b; 2005; Youn et al., 2004; 2005; Choi et al., 2004; Huang et al., 2009; 2012b; Zhang et al., 2010b). Fuzzy reliability theory in the context of possibility theory is proposed and developed by (Cai et al., 1991a; 1991b; 1993; Utkin and Gurov, 1996; Onisawa, 1988; Huang et al., 2004; 2010). In addition to reliability engineering, the application areas also include civil engineering and structural engineering (Moller et al., 1999; Huang et al., 2011a; Huang, 2012), computational mechanics, military, energy, forestry (Kangas and Kangas, 2004), aerospace and automobile engineering (Cayrac et al., 1996) and many other fields.

As a more general tool for uncertainty analysis, evidence theory has also been applied to many areas, including artificial intelligence (particularly in the development of expert systems) (Bae et al., 2004b; Nikolaidis and Haftka, 2001), object detection and approximate reasoning (Lowrance et al., 1986; Perrin et al., 2004; Xu and Smets, 1996; Borotschnig et al., 1999), design optimization (Mourelatos and Zhou, 2005), multidisciplinary design optimization (Agarwal et al., 2004), uncertainty quantification (Bae et al., 2004a; 2004b), risk and reliability evaluation (Yang et al., 2011b), remote sensing classification (Lee et al., 1987), pattern recognition and image analysis, decision making (Buckley, 1988; Limbourg, 2005), data fusion (Delmotte and Borne, 1998; Hall and Llinas, 1997; Sun et al., 2008; Yang et al., 2011a) and fault diagnosis (Fan and Zuo, 2006a; 2006b; Wu et al., 1990). The popularity of evidence theory has risen, however, because evidence theory requires epistemological assumptions that are at odds with those underlying classical and Bayesian probability theories (Fioretti, 2004).

There is a tendency to use more than one framework to deal with complicated and variable environments. Work of this kind include the integration of probabilistic and possibilistic approaches (Youn and Choi, 2004a; Youn et al., 2004), probabilistic integration of the probabilistic and evidential approaches (Lee et al., 1987); integration of possibility-based design optimization and robust design (Youn et al., 2005; Huang et al., 2009), integration of probabilistic optimization and robust design (Du et al., 2003), integration of aleatory and epistemic uncertainty for various design optimizations (Mourelatos and Zhou, 2005; Huang and Zhang, 2009; Zhang and Huang, 2010; Huang et al., 2012a) and so forth.

From our point of view, the existing applications of and developments in possibility and evidence theories deal with uncertainty and reliability analysis, mainly focusing on two aspects. One is theoretic development related to the fundamentals of reliability theory, e.g. imprecise reliability (Walley, 1991; Utkin and Coolen, 2007; Kozine and Filimonov, 2000) and fuzzy reliability (Cai et al., 1991a; 1991b; 1993; Huang et al., 2004; 2010); the other is computational (or algorithmic) development in analysis and the design method, e.g., data fusion technology applied to reliability assessment (Hall and Llinas, 1997; Zhang et al., 2010a; Sun et al., 2008; Yang, 2011a; 2011b) and optimum design methods (Youn and Choi, 2004b; Youn et al., 2004; Aughenbaugh and Paredis, 2005; Huang et al., 2005a; Limbourg, 2005; Mourelatos and Zhou, 2005; Huang et al., 2006a; 2006b; 2012a). These are illustrated in the sections that follow.

The two aspects of applications and developments arise from the variety and complexity in engineering environments, i.e.:

- Various types of uncertainty are taken into account (Youn, 2005; Wu, 2008; Wu et al., 2002; Huang et al., 2012b; Zhang et al., 2010b) 
- Lack of information in input data, small sample size, insufficient information or scarce data (Chen et al., 1999; Mourelatos and Zhou, 2004; Nikolaidis and Haftka, 2001; Huang et al., 2004; 2010)

- Imprecise data or lack of knowledge (Choi et al., 2004; Youn and Choi, 2004a; 2004b; Youn et al., 2004; Aughenbaugh and Paredis, 2005; Huang et al., 2005b; 2006d; Limbourg, 2005; Mourelatos and Zhou, 2005)

- Large-scale, complex or costly systems (Zhuang et al., 2000)

\subsection{Fundamental Developments Related to Reliability Theory}

Reliability research was initiated by the problem of machine maintenance in the late 1930s and of replacing street-lighting lamps in the early 1940s; it was given an especially impetus by the demands of complex systems in World War II (Cai et al., 1991a; 1991b). The word reliability is concerned with whether a system can operate properly without failure. It is defined as the ability of an item to perform a required function under stated conditions for a stated period of time. The term can also be denoted as a probability or as a success ratio or feasibility. Thus, in most cases, reliability is connected with such related concepts as failure and loss of quality.

Classifying aspects of reliability is subject to various considerations, such as general topics of reliability (reliability engineering, reliability management, warranty and maintenance), contents of reliability research (reliability assessment, reliability prediction and modeling, reliability analysis, reliability allocation and reliability testing), stages of reliability (reliability in design, reliability in manufacture, reliability in use), objects of reliability (hardware reliability, software reliability, human reliability, structural reliability) among other criteria.

At present, the chief reliability design activities involve Fault Tree Analysis (FTA), FMEA and reliability optimization.

Conventional reliability analysis of engineering systems relies on a probabilistic method, which represents the system state variable as precise probability distributions and generates precise estimations of system failure given sufficient input data. In complicated situations of engineering decision making, we encounter many indeterminable factors, both during the early stages of design and in the process of a product's manufacturing and use; these are due to lack of knowledge. A determinate decision from a precise probabilistic method of analysis can not be considered a faithful reflection of reality itself. In reliability practice, there have recently been some fundamental theoretic developments involving safety and reliability analysis when data is scarce or incomplete.

\subsubsection{Imprecise Reliability}

\subsubsection{Origin and Basics of Imprecise Probability Theories: Origin and Motivation}

It is popularly accepted that engineering design is a process of decision making during which engineers, because they inherently don't have enough information, must deal with uncertainty. Only if the imprecise characterizations of uncertainty are accommodated, can it be said that the uncertainty is reflected or represented clearly and quantitatively. Imprecision can result from fundamental indeterminacy in the available evidence or from incomplete characterizations of the available evidence or beliefs (Youn et al., 2004).

Imprecise probabilities have been used to represent uncertainty in practical reliability and risk analysis, by characterizing state-of knowledge uncertainty with intervals of probabilities. The general motivation for imprecise probabilities is that the confidence felt by a decision-maker depends mainly on the evidence on which his/her probability estimate is based. Thus, any imprecision in the probabilities should be expressed explicitly in order to signal the level of confidence appropriate to them (Youn et al., 2004).

There are several theories of imprecise probabilities, including evidence theory and possibility theory. Probability theory, possibility theory and evidence theory are all special cases of fuzzy measure theory and recently, a theory of coherent imprecise probabilities has been developed by Walley (1991) and Wu et al. (1990).

The term imprecise probabilities is used as a generic one to cover mathematical models such as upper and lower probabilities, upper and lower previsions (or expectations), possibilities and necessities, belief and plausibility functions and other qualitative models (Kozine and Filimonov, 2000).

The coherent imprecise probability theories (Walley, 1991) are based on a behavioral interpretation and three fundamental principles: avoiding sure loss, coherence and natural extension. The basic concept associated with behavioral interpretation is that of a gamble. This is a bounded real-valued function defined on domain $\Omega$ and it should be interpreted as a reward whose value depends on an uncertain state. Each one 
belongs to the domain $\Omega$, in the context of decision theory and utility theory.

The coherent imprecise probability theories are also based on two probabilistic models: Lower previsions (or expectations) and upper previsions. In reliability and risk analysis problems, we consider a particular gamble for which the reward can be either 0 or 1 . In this case, the lower and upper previsions are called lower and upper probabilities, respectively, just as the name implies.

The combinative rules of multi-source information discriminate between consistent and inconsistent judgments (or models). The former contain the conjunction rule, which combines lower and upper previsions for consistent judgments. The latter include an alternative rule called the unanimity rule. Walley (1991) obtained these on the basis of concept desirability and preference.

\subsubsection{Application and Advances of Imprecise Probability Theories}

Imprecise probability theories, including evidence theory and possibility theory, have proved to be useful and applicable to the implementation of reliability and risk analysis, even though some unsatisfactory points have been criticized (e.g., difficulties with evidence's combinations, diversity of judgments admitted in elicitation) Kozine and Filimomov (2000) developed imprecise probabilities as a particularly advantageous way of handling indeterminacy and summarized their experiences in dealing with evidence theory as it relates to reliability assessments. Using practical system reliability assessments for serial, parallel and general reliability structures, they demonstrated recent advances in applying the theory of coherent imprecise probabilities to system reliability assessments.

Walley (1991) and $\mathrm{Wu}$ et al. (1990) proposed a theory of coherent imprecise probabilities with a behavioral interpretation in terms of decision theory and utility theory.

Hall and Lawry (2001) introduced a new method of constructing an imprecise limit state function from scarce data based on minimal assumptions about the underlying systems behavior. Application to a case study on reliability analysis has demonstrated how this conventional approach can be extended to handle imprecise knowledge about system state variables. It is represented in general as random sets, in order to generate bounds on the probability of failure. This approach has provided new insights into the sources of uncertainty and the assumptions implicit in the conventional probabilistic approach.

Coolen (2004) discussed a variety of issues involving advantages and disadvantages and reviewed suggested applications of imprecise probability in reliability. A recently developed statistical approach, called nonparametric predictive inference to reliability, has been introduced by Coolen et al. (2002) as a coherent framework offering exciting opportunities for when data is scarce. They also presented applications of this approach with regard to replacement and maintenance decisions.

Aughenbaugh and Paredis (2005) consider imprecise probabilities in order to express clearly the precision with which something is known, on the hypothesis that it is valuable to explicitly represent this imprecision by using imprecise probabilities in engineering design. Then an example and computational experiments involving pressure vessel design problems are carried out using two approaches, both variations of utility-based decision making. The experiments demonstrate that when designers have access to only a small set of sample data, a Probability Bounds Analysis (PBA) approach that uses imprecise probabilities to model uncertainty can lead on average to better designs than can a purely probabilistic approach that requires precise probabilities.

Augustin and Hable (2010) claim that building a relationship between the theory of imprecise probabilities and robust statistics is promising. Oberguggenberger et al. (2009) have applied imprecise probability to deal with sensitivity analysis. An aerospace engineering example is used to compare the results obtained using random sets, fuzzy sets and interval spreads simulated with the aid of the Cauchy distribution.

We can conclude from these results that, at least in some design problems, it is valuable to explicitly express any imprecision in the available characterization of uncertainties in terms of imprecise probabilities. Further introductory information and examples of imprecise reliability analysis can be found in (Nikolaidis and Haftka, 2001).

Although applying imprecise probability methods to reliability has shed light on many interesting research problems, there is still a need for a wide variety of research tools for addressing problems. By far, the main difficulties for modeling imprecise probabilities involve computation. Recent work by (Utkin and Coolen, 2007) has made great progress in this regard, yet much remains to be done. Another topic that has not yet been studied is the design of experiments with uncertainty quantified through imprecise probabilities (Youn et al., 2004; Nikolaidis and Haftka, 2001). From this perspective, the wide backgrounds of development for researchers 
devoted to this field may be of great benefit in future.

\subsubsection{Fuzzy Reliability}

Similar to the origin of imprecise probability theories, possibility theory and evidence theory also find their positions in reliability analysis. The systematic and typical one which has been well-developed is fuzzy reliability. Mainly referred to as employing possibility theory, one uses fuzzy theory as the uncertainty analysis tools in the framework of reliability theory.

\subsubsection{Motivation and Consideration of Fuzzy Theory in Reliability Analysis}

The origin of fuzzy reliability theory comes from the consideration of reliability aspects in gracefully degradable computing systems, where system states cannot be simply classified as failed or functioning. In addition to the nature of performance degradation, a failure does not necessarily occur at random because of complex and uncertain factors. Hence, a concept of fuzzy reliability was proposed by to meet these demands.

In detail, the two basic assumptions of traditional probability-based reliability analysis, is not appropriate if the data to estimate the failure probability is not enough; or the determination of a safety criterion is also dependent on engineering judgment, which lead to diverse uncertain factors. Utilizing emergence and development of uncertain quantification analysis tools, such as possibility theory and evidence theory, the concept of fuzzy reliability was introduced based on possibility theory (Cai et al., 1991a; 1991b; 1993).

\subsubsection{Classifications and Structures of Fuzzy Reliability Theory}

It is no surprise to note that possibility theorybased reliability theory can be classified as not only a member of fuzzy reliability theory, but also a member of non-probabilistic or imprecise probability theory. The former classification is due to the theoretic background of fuzzy set theory while the latter classification is due to the non-statistical characteristics of the information involved. Compared with the conventional reliability theory, the structure of fuzzy reliability theory is illustrated as Fig. $\mathbf{6}$.

\subsubsection{Present Works on the Subject of Fuzzy Reliability}

According to different reliability assumptions, various forms of fuzzy reliability theories, including profust reliability theory, posbist reliability theory and posfust reliability theory, have been considered to put new assumptions in place of the binary-state and probability assumptions.

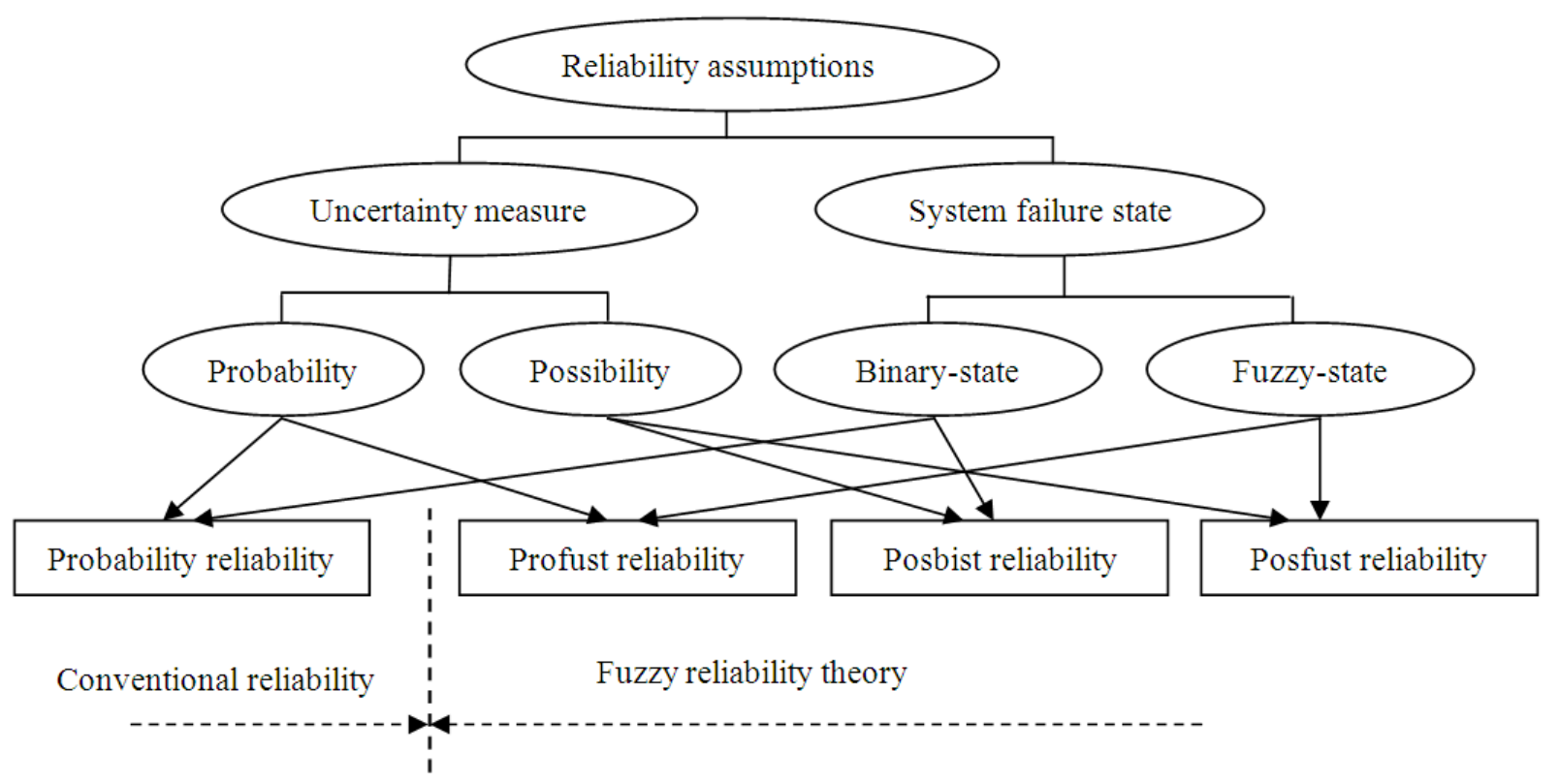

Fig. 6. Reliability theories based on various fundamental assumptions 
With the concept of possibilistic logic proposed by (Dubois and Prade, 1983; 1987), a new knowledge-based solution now enables possibility theory to achieve wider applications in the artificial intelligence or data fusion domains than was possible with probability theory and evidence theory.

To date, most existing works are in theoretic construction and modification (Cai et al., 1991a; 1991b; 1993; 1995a; 1995b; Cappelle and Kerre, 1993; 1994; 1995b; Moller et al., 2004; Nikolaidis et al., 2004; Huang et al., 2010), or practical connections in engineering (Cappelle and Kerre, 1995a; 1995b; Bae et al., 2004a; 2004b; Bai and Asgarpoor, 2004; Moller et al., 1999; 2004; Li et al., 2012). Cai et al. (1991a; 1991b) has considered Posbist reliability theory for typical systems, such as series, parallel and k-out-of-n systems as well as for cold and warm redundant systems (Cai et al., 1995a; 1995b). Utkin (1994) and Utkin et al. (1995) has provided an analysis of typical repairable systems in the possibility context. Aiming at more change-friendly systems, Utkin and Gurov (1996) proposed a general formal approach to analyzing posbist reliability behavior in arbitrary systems using a state transition diagram. Systematic work on maintenance policy and FTA in the presence of fuzzystate assumptions has been only been partially done. Huang et al. (2004) have developed a new model of fault tree analysis corresponding to posbist reliability theory in order to evaluate system reliability and safety when statistical data is scarce or the probability of failure is extremely small.

Most recently, fuzzy reliability has been extended to a multi-state systems context where both components and systems possess multiple discrete performance rates in lifecycle (Liu et al., 2008; Liu and Huang, 2010; 2011) and dynamic fault tree analysis (Li et al., 2012).

Although much research has been done on fuzzy reliability theory itself and its extensions, the exploration is not complete yet. Existing models of fuzzy reliability theory have caused some difficulties for practitioners who feel this theory does not cover a large enough variety of possible judgments in reliability (Utkin and Coolen, 2007). Actually, even the evidence-theory-based approach to reliability analysis encounters this criticism when information is incomplete. In some real cases, there does not exist a type of possibility distribution that is reasonably consistent with statistical data. Clear interpretation of possibility distributions is a goal for the future.

To a certain extent, reliability analysis using the proposed possibility-based method can not be completely separated from possibility-based design optimization. Nikolaidis et al. (2004) and Nikolaidis and Haftka (2001) compared the theoretical foundations of probability- and possibility-based methods in design for maximum safety and concluded that possibility theory tends to be less conservative than probability theory in risk assessment when data is sufficient and to be more conservative under more unfavorable circumstances that is under epistemic uncertainty.

\subsection{Computational Developments Related to Design Optimization Under Uncertainty}

\subsubsection{Design Optimization}

\subsubsection{An overall Understanding of Design Optimization}

Design optimization is now a mainstream discipline in high-technology product development and a natural extension of the ever-increasing analytical abilities of computer-aided engineering (Papalambros and Michelena, 2000). Such factors as designing largescale complex systems, business demands and technological intersections, all require methods of design optimization with a system view, in contrast to a view of component design.

Design optimization under uncertainty should take into account the main types of uncertainty arising during the design or operation of the product, i.e. physical of uncertainties (manufacturing tolerances, uncontrollable variations in external operating conditions) and uncertainties in decision making (vagueness in conflicting objectives), uncertainties in modeling and simulation.

\section{(1) Formal Mathematical Model of Design Optimization}

Design optimization assumes a decision-making paradigm for the design process. It takes the following form expressed as Equation 52:

$$
\begin{array}{ll}
\text { minimize } & \mathrm{f}(\mathrm{d}, \mathrm{X}) \\
\text { subject to } & \mathrm{h}(\mathrm{d}(\mathrm{X}))=0 \\
& \mathrm{~g}(\mathrm{~d}(\mathrm{X})) \leq 0 \\
& \mathrm{X}_{\mathrm{i}} \in \mathrm{U} \subseteq \mathrm{R}^{\mathrm{n}}
\end{array}
$$

where, $f(*)$ is the scalar design objective function, $d=$ $[\mathrm{di}]^{\mathrm{T}}$ is the vector of design variables in the n-dimensional real space $R^{n}, X=\left[X_{i}\right]^{T}$ is the vector of random variables, the vector-valued functions $\mathrm{h}\left({ }^{*}\right)$ and $\mathrm{g}\left({ }^{*}\right)$ are the constraint functions that determine whether a design is feasible (reliable) or not. In a practical engineering optimization problem, the main criteria used to measure the effectiveness are cost and performance. 
Although providing a practical tool for analysis and design, the traditional design optimization has its drawbacks because it does not consider the inherent uncertainties (such as variations in design variables and parameters) and modeling uncertainties (such as modeling and numerical errors existing in the analysis tool) quantitatively (Dhanesh, 2003). Hence researchers have proposed various specialized optimization methodologies to reduce the computational costs of traditional design optimization problems. There have been some advances in exploring decomposition strategies or approximation concepts, especially in aerospace and automobile engineering applications.

\section{(2) Classifications of Design Optimization}

The diversity of system structures, resource constraints and types of uncertainty considered has led to the construction and analysis of various design optimization models (or design methods under uncertainty) (Liang et al., 2008; Huang et al., 2012a; 2012b; Huang et al., 2011b; Huang and Zhang, 2009; Huang et al., 2008):

- Reliability-Based Design Optimization (RBDO): aleatory uncertainties

- Possibility-Based Design Optimization (PBDO): epistemic uncertainties

- Evidence-Based Design Optimization (EBDO): epistemic uncertainties, also for a mixture of aleatory and epistemic uncertainties

- Interval-Based Design Optimization (IBDO): interval variables for describing design variables or/and parameters with two bounds

- Robust design: mainly aimed at enhancing product quality as well as reliability

- Design for six-sigma: satisfy the six-sigma requirements under uncertainty, mainly a combination of reliability-based design optimization and robust design

Traditional design optimization methods can be classified as a bi-level approach. When concrete uncertainty-based design optimization is applied, the methods can be categorized as:

- Double-loop method: Two loops (Inner loop: reliability analysis and outer loop: design optimization) are nested, which is the simple method but with low computational efficiency (Zhang and Huang, 2010)

- Single-loop single-variable method: It is mainly proposed for the RBDO process and attempts to improve numerical efficiency by eliminating numerical iterations in the only loop of reliability analysis (Du et al., 2003)

- Safety-factor approach: The approximate equivalent deterministic constraints are used to decouple the optimization and reliability analysis

- Sequential optimization and reliability assessment: A series of cycles are obtained by decoupling the deterministic optimization and reliability assessment in order to improve computational efficiency $(\mathrm{Tu}$ and Choi, 1999; Du and Chen, 2004; Zhang and Huang, 2010; Huang et al., 2012a)

Based on types of principles and theories of uncertainty, there is a classification of analysis methods for uncertain system in the present literature (Langley, 2000; Choi et al., 2004) as follows.

Describing uncertain input variables via a probability density function. The well-known probabilistic approaches include.

\section{1) Asymptotic Reliability Analysis}

It fully describes the statistics of the engineering structure system by the joint probability density function with the random variables in the form of the entries of a vector and dimension. The condition of the structure is described by a safety margin. Here the word asymptotic means that the errors involved in the approximation of failure probability approaches zero when the reliability index goes to infinite.

\section{2) First-Order Reliability Method (FORM)}

It is a subset of the asymptotic approach by transforming the random variables to a set of uncorrelated Gaussian variables with zero mean and unit variance and the safety margin is approximated by a hyperplane.

\section{3) Second-Order Reliability Method (SORM)}

It follows the identical steps of FORM analysis except that the final representation of the safety margin uses a quadratic approximation.

\section{4) Other Methods}

Such as Monte Carlo Simulation (MCS) technology, Bayesian method, experimental design techniques (or Taguchi's method).

\section{1) Interval Analysis}

Expressing uncertain input variables via lower and upper bounds and defining the output interval by the 
minimum and maximum of all input end point combinations (Youn and Choi, 2004a).

\section{2) Convex Modeling}

Assuming that uncertain input variables lie within a convex region and reducing to interval analysis when taking a hypercube convex region.

\section{3) Fuzzy Modeling (or Possibility Theory Using a Fuzzy Set)}

Nikolaidis et al. (2004) and Nikolaidis and Haftka (2001): Describing uncertain input variable by a fuzzy number (or possibility distribution function).

Encompassing or integrating the probabilistic and possibilistic analysis methods in a general framework, e.g., a common mathematical algorithm in reliability analysis (Langley, 2000).

\section{(3) General Introduction of RBDO and its Development}

Reliability-Based Design Optimization (RBDO) has been used to consider aleatory uncertainties in an engineering design process. When the input data contain sufficient information to characterize statistical distributions, the design optimization that incorporates the probability method is called a reliability-based design optimization (Youn et al., 2004). The modern reliability methods themselves are actually formulated as a problem of optimization, which involves evaluating of probabilistic output performance measures.

The standard RBDO model can be defined as Equation 53 (Youn et al., 2004), by replacing the hard constraints of traditional design optimization with reliability constraints:

$$
\begin{array}{ll}
\min & \operatorname{Cost}(d, X) \\
\text { s.t. } & P\left(G_{i}(d(X))>0\right) \leq \Phi\left(\beta_{t_{i}}\right), \quad i=1,2, \cdots, n p \\
& d^{L} \leq d \leq d^{U} \\
& d=\mu(X) \in R^{n} \text { and } X=\left[X_{i}\right]^{T} \in R^{n r}
\end{array}
$$

where, $\mathrm{d}$ and $\mathrm{X}$ are the design vector and the random vector respectively while $\beta_{\mathrm{t}}$ is a target failure probability (or reliability level) and n, nr, np are the number of design variables, the number of random variables and the number of probability constraints, respectively. The design variables can be distribution parameters like means or standard deviations of the random variables, or of course can be deterministic. The reliability constraints are on the reliabilities with respect to the various failure modes.

Some alternative methods (Dhanesh, 2003) have been suggested to reduce the high computational costs related to a traditional RBDO, including a unified-level RBDO, Performance Measure Approach (PMA), semiinfinite optimization, a single level approach and a variable complexity algorithm. In a unified-level RBDO, the FORM-based reliability constraints take the place of the first order optimality conditions of the Most Probable Point (MPP) searches. In a PMA, the FORM-based reliability constraints are replaced with the worst case values of hard constraints under a specified reliability level. In a semi-infinite optimization techniques based on PMA, the constraints are replaced with a single deterministic one in form of a min-max formulation, satisfying the hard constraints within a sphere on radius of required reliability.

The approximation concepts in RBDO can improve the efficiency of such approaches, including Two-point Adaptive Nonlinear Approximation (TANA2), multivariate spline approximation. A more detailed survey of detailed approximation and optimum design can be found in (Dhanesh, 2003).

Recently, the extensive research or development of RBDO concentrates on how to make it computationally affordable, while maintaining numerical accuracy and stability. Tu and Choi (1999) have reformulated the FORM reliability constraints by an inverse reliability analysis formulation. They also point out that PMA can work for cases where conventional MPP searches fail. (Youn and Choi, 2005) present an enriched Performance Measure Approach (PMA+) for RBDO to substantially improve computational efficiency in large-scale applications, by carrying out the refined reliability analysis using the enhanced Hybrid Mean Value $(\mathrm{HMV}+)$ first-order method. We can find that such approach can also be applied and developed in a PBDO environment, with each method having its own strong and weak points (Mourelatos and Zhou, 2005). In addition to these methods, a new reliability analysis tool based on Trust Region methods is also developed.

On the other hand, a general optimization under uncertainty formulation can adopt both robustness and reliability constraints. One such formulation that minimizes both mean of merit function and its variance subject to constraints on worst value of hard constraints within intervals of required confidence level is expressed as Equation 54 (Su and Renaud, 1997):

$$
\begin{array}{ll}
\min & \mu_{\mathrm{f}}+\mathrm{w \sigma}_{\mathrm{f}} \\
\text { s.t. } & \mu_{\mathrm{g}_{\mathrm{i}}}-\beta_{\mathrm{t}} \sigma_{\mathrm{g}_{\mathrm{i}}} \geq 0 \quad \mathrm{i}=1,2, \cdots, \mathrm{N}_{\text {hard }} \\
& \mathrm{d}^{\mathrm{L}} \leq \mathrm{d} \leq \mathrm{d}^{\mathrm{U}}
\end{array}
$$

where, $\mu_{\mathrm{f}}$ and $\sigma_{\mathrm{f}}$ are the mean and standard derivation of variables and $\mathrm{w}$ is a weighting function. 


\subsubsection{PBDO}

We have mentioned earlier that in practical engineering design, there are two main types of uncertainty: Aleatory and epistemic uncertainties. The former is objective and irreducible with a large amount of information on input data and then can be modeled using probability theory. The latter is subjective and reducible coming from lack of knowledge on input data.

In areas where it is not possible to obtain accurate statistical data due to restriction of resources or conditions (i.e., budgets, facilities, time, human factors), probabilistic methods may not be appropriate for structural analysis and design optimization. Thus a demand for alternative design methods is created by epistemic uncertainty which requires the modeling of physical uncertainty when there is insufficient information (Klir, 2000; Youn et al., 2004). Possibility theory and evidence theory are used in such cases. Very recently, possibility-based (or fuzzy set) methods have been proposed (Klir, 2000; Choi et al., 2004; Moller et al., 2004; Zhang et al., 2010b), in which a mean performance is optimized subject to possibilistic constraints.

\section{(1) Formation of PBDO}

The general PBDO can be formulated as Equation 55 (Zhou and Mourelatos, 2008):

$$
\begin{array}{ll}
\min & \operatorname{Cost}(d, Y) \\
\text { s.t. } & \Pi\left(G_{i}(d(Y))>0\right) \leq \alpha_{t_{i}}, \quad i=1,2, \cdots, n p \\
& d^{L} \leq d \leq d^{U} \\
& d=\mu(Y) \in R^{n} \text { and } Y=\left[Y_{i}\right]^{T} \in R^{n r}
\end{array}
$$

where, $\mathrm{d}$ and $\mathrm{Y}$ are the design vector and the fuzzy random vector respectively while $\alpha_{t}$ is a target failure possibility and $\mathrm{n}, \mathrm{nr}, \mathrm{np}$ are the number of design variables, the number of fuzzy random variables and the number of possibility constraints, respectively.

\section{(2) Fuzzy Analysis Method for PBDO}

Compared to other methods, a fuzzy (or possibility) analysis method represents a very useful tool to perform operations in the framework of possibility theory, with the following main advantages: (1) preserving the intrinsic random nature of physical variables through their membership functions; (2) simpler extended fuzzy operations (Klir and Folger, 1988; Klir and Yuan, 1995) than those required to use probability; (3) yielding a more conservative design than the probabilistic design method in terms of a confidence level (Klir, 2000); (4) providing a system-level possibility unlike reliability analysis.

For numerical methods of fuzzy analysis, some reported methods are listed below:

- $\quad$ Vertex method (Sentz and Ferson, 2002)

- Discretization method

- $\quad$ Level-cuts ( $\alpha$-cuts) method (Huang et al., 2008)

- Multilevel-cut method

- Possibility index approach

- $\quad$ Performance Measure Approach (PMA)

- Most Probable Point (MPP) search

- Maximal Possibility Search (MPS)

In practical engineering analysis and design, the vertex method is popular but rather expensive for large-scale engineering applications and could yield inaccurate results of fuzzy analysis in the case that an output response has a maximum or minimum within the input range. A levelcuts method has been used to overcome the difficulties of nonlinear problems using various design levels. Recently, a multilevel-cut method has been developed to improve the accuracy of the vertex method for nonlinear structural design, but it is also very expensive to carry out PBDO. The PMA has been successfully applied with its advantages of numerical efficiency and stability in PBDO (Youn et al., 2003; Youn et al., 2004).

Fuzzy analysis method is different from reliability analysis in such two cases: one is the MPP in reliability analysis based on FORM results in the first order approximation, whereas MPP in fuzzy analysis is exact along with the related possibility; the other is that the search domain is different, which is an nr-dimensional sphere in reliability analysis while nr-dimensional hypercube in fuzzy analysis as shown in Table 6, thus lead to simpler computation in fuzzy analysis (Choi et al., 2004).

\section{(3) Comparison of PMA in PBDO and RBDO}

Both RBDO and PBDO employ PMA to improve numerical efficiency, stability and accuracy. The difference of PMA method in reliability analysis by (Youn et al., 2004; 2003) and in fuzzy analysis (Cai et al., 1991a; 1991b; 1993; 1995a; 1995b; Cappelle and Kerre, 1993; 1994; 1995a) is illustrated in Table 6.

\section{(4) Present Works Related to PBDO}

At present, one of the main concerns related to PBDO research has been how to improve numerical efficiency, accuracy and stability during the optimization process. The Performance Measure Approach (PMA) is such a method satisfying the requirement, replacing the probabilistic constraint in Equation 53 with the performance measure under a specified reliability level (Youn and Choi, 2005; Youn et al., 2003). 
Table 6. Comparison of PMA method in RBDO and PBDO (Choi et al., 2004; Youn et al., 2004; Youn, 2005)

\begin{tabular}{|c|c|c|}
\hline & PMA in RBDO & PMA in PBDO \\
\hline & $\min \operatorname{Cost}(\mathrm{d} ; \mathrm{X})$ & $\min \operatorname{Cost}(\mathrm{d} ; \mathrm{Y})$ \\
\hline Formulation & s.t. $\quad \mathrm{G}_{\mathrm{pi}}(\mathrm{d}(\mathrm{X})) \leq 0, \quad \mathrm{i}=1,2, \cdots, \mathrm{np}$ & s.t. $\mathrm{G}_{\pi \mathrm{i}}(\mathrm{d}(\mathrm{Y})) \leq 0, \quad \mathrm{i}=1,2, \cdots, \mathrm{np}$ \\
\hline \multirow[t]{2}{*}{ Objective of constraints } & $\begin{array}{l}\quad \mathrm{d}^{\mathrm{L}} \leq \mathrm{d} \leq \mathrm{d}^{\mathrm{U}} \\
\text { random quality loss and random } \\
\text { manufacturing cost. }\end{array}$ & $\begin{array}{l}\quad \mathrm{d}^{\mathrm{L}} \leq \mathrm{d} \leq \mathrm{d}^{\mathrm{U}} \\
\text { Deterministic material cost, random quality loss } \\
\text { manufacturing cost. }\end{array}$ \\
\hline & $\mathrm{G}_{\mathrm{pi}}:$ the $i$ th probabilistic constraints & $\mathrm{G}_{\pi \mathrm{i}}:$ the $i$ th possibilistic constraints \\
\hline \multirow{3}{*}{ Evaluation of constraints } & $\min G(U)$ & $\min G(V)$ \\
\hline & s.t. $\|\mathrm{U}\|_{2} \leq \beta_{\mathrm{t}}$ & s.t. $\|\mathrm{U}\|_{\infty} \leq 1-\alpha_{\mathrm{t}}$ \\
\hline & $\begin{array}{l}X: \text { random variable } \\
\text { U: standard normal random variable; } \\
\text { d: Design variable. } d=\mu(X) \in R^{n} \text {. }\end{array}$ & $\begin{array}{l}\text { Y: non-interactive fuzzy variables; } \\
\text { V: fuzzy variable with isosceles } \\
\text { d: Design variable, }\end{array}$ \\
\hline \multirow{2}{*}{ Variables and parameters } & $\beta_{\mathrm{t}}:$ target reliability index or target & $\mathrm{d}=\left\{\max \left[\Pi_{\mathrm{Y}_{\mathrm{i}}}\left(\mathrm{y}_{\mathrm{i}}\right)\right]\right\}=\left[\mathrm{d}_{\mathrm{i}}\right]^{\mathrm{T}}$ \\
\hline & reliability level & $\alpha_{\mathrm{t}}$ : target failure possibility. \\
\hline
\end{tabular}

Choi et al. (2004) provided a new formulation of PBDO using PMA to improve numerical efficiency, stability and accuracy. They also propose a new Maximal Possibility Search (MPS) method to resolve disadvantages of the vertex method and the multilevel-cut method, by evaluating possibility constraints efficiently and accurately for nonlinear structural applications. Youn et al. (2004) presented an integrated design platform of both RBDO and PBDO using PMA when modeling physical uncertainty with insufficient information. (Mourelatos and Zhou, 2004) used the possibility theory as a variant of fuzzy set theory to assess reliability with incomplete information in structural analysis and design.

In their study, a hybrid optimization approach for calculation of the confidence level of fuzzy response is presented first, combining the merits of conventional vertex and discretization methods. Then they provide a general PBDO method with numerical examples, which is from the angle of design and proves to be computationally efficient. Tu and Choi (1999) showed that the advantage of PMA is that when the reliability index is very high, PMA is less expensive and the disadvantage of this approach is that it might require more computations where the reliability index is lower than the required reliability level. So there is a need for some modification and extension of fuzzy analysis methods in PBDO.

In order to improve the computational efficiency and stability, the enriched Performance Measure Approach (PMA+) has been proposed. As an extension of PMA, it combines four key ideas (Youn and Choi, 2005): as a way to launch RBDO at a deterministic optimum design, as a probabilistic feasibility check, as an Enhanced Hybrid-Mean Value $(\mathrm{HMV}+)$ method and as a fast reliability analysis under the condition of design closeness. Youn and Choi (2004a) provided two examples to show computational features of PMA+ for RBDO with aleatory input uncertainties and MPS for PBDO with epistemic input uncertainties. In addition, RBDO and PBDO results are compared for implications of these methods in design optimization.

Another concern of the extension of PBDO is to provide a general framework integrating various proposed design optimization methodologies such as RBDO, PBDO, under aleatory uncertainty or epistemic uncertainty, or both of them. Youn (2005) proposed a method called the adaptive-loop method used for such integrated framework, enhancing numerical efficiency without losing computational stability by integration of parallel-loop and single-loop methods adaptively.

Nikolaidis et al. (2004); Nikolaidis and Haftka (2001) and Chen et al. (1999) also consider the PBDO problem for design under uncertainty. It is shown that more conservative results are obtained compared with the probability-based RBDO, especially when there is insufficient information available in reliability assessment. But it is also true that using possibility theory can yield less conservative designs in certain cases, when the main design criterion is to minimize the probability and possibilities of failure.

The existing works related to PBDO have revealed the characteristics and advantages of possibility theory for coherent systems; but there still need a general solution (not referred to only method or algorithms) of all uncertainty-based optimization under diverse uncertainties, which could be the future direction of research. 


\subsubsection{EBDO}

As a more general tool for uncertainty quantification analysis than probability and possibility theory, evidence theory has shown its qualitative value and computational efficiency in many application areas.

The significant point of evidence theory is that it allows for allocation of a probability mass to sets or intervals and does not require a premier assumption regarding the probability of the individual constituents of the set or interval, compared with other methods. These special advantages are potentially valuable in engineering design if limited and even conflicting information is provided from expert elicitation or experiments, by means of combining aleatory and epistemic uncertainty in a straightforward way.

However, to the best of our knowledge, reported exploration of evidence theory in engineering design is fairly limited and even much less in a design optimization framework. It is only recently that evidence-based methods are used to propagate epistemic uncertainty (Bae et al.,
2002; Bae et al., 2004a), e.g., in large-scale engineering structure systems. One of the major difficulties of applications for evidence theory, investigated by Bae et al. (2004a; 2004b) may be its high computational cost. Adopts evidence theory as a general tool of uncertainty quantification analysis for large-scale structures, using a cost-effective and reliable algorithm, the Multi Point Approximation (MPA) to alleviate the computational difficulties (Bae et al., 2002; Bae et al., 2004a; 2004b). In their study, compared with the popular evidence-based methods of the sampling method and the vertex method, such a proposed optimization technique as MPA method enhances its accuracy mainly through local approximation, focusing the computational recourses on the failure region and then the Two-point Adaptive Non-linear Approximate (TANA2) is selected. Both the optimization and the approximation techniques may efficiently evaluate the belief and the plausibility functions without sacrificing the accuracy of resulting measurements. The detailed flow diagram is showed in Fig. 7.

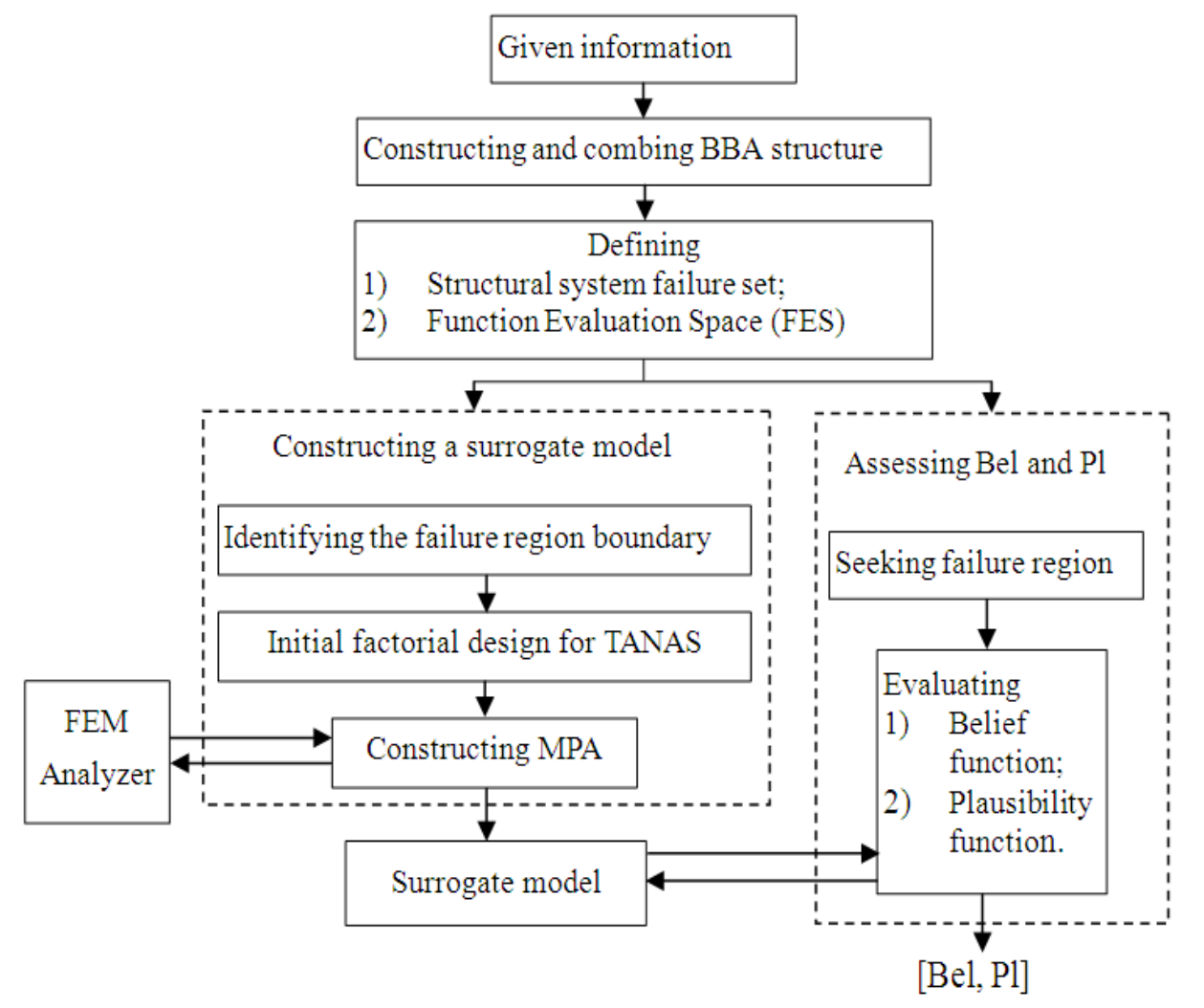

Fig. 7. An uncertainty qualification approximation algorithm using evidence theory (Bae et al., 2004a; 2004b) 
Although such a computationally efficient and accurate method is proposed and demonstrated by two structural examples (Bae et al., 2004a), it is not an issue which takes design problem into account. The study which propagates epistemic uncertainty using evidence theory and also performs a design optimization is first carried out by (Agarwal et al., 2004), in which optimum design is calculated for multidisciplinary systems under uncertainty. Since the belief functions are discontinuous to formulate non-deterministic constraints in this research, Agarwal et al. (2004) employ a trust region sequential approximate optimization method to drive the optimization process with surrogate models representing the uncertain measures as continuous functions. Their work is significant in throwing light on the use of evidence theory for optimization under uncertainty.

Mourelatos and Zhou (2005) continue the research of Evidence-Based Design Optimization (EBDO). The proposed design optimization method, which is computationally efficient and can handle a mixture of aleatory and epistemic uncertainties, can be formulated as Equation 56:

$$
\begin{array}{cc}
\min & f\left(d, X^{N}, P^{N}\right) \\
\text { s.t. } & P l\left(G_{i}(d(X))>0\right) \leq p_{f_{i}}, i=1,2, \cdots, n p \\
& d^{L} \leq d \leq d^{U} X_{L}^{N} \leq X^{N} \leq X_{U}^{N} \\
& d \in R^{n}, X \in R^{n r}, P \in R^{q}
\end{array}
$$

where, $\mathrm{d}, \mathrm{X}$ and $\mathrm{P}$ are the vectors of deterministic design variables, uncertain design variables and uncertain design parameters, respectively. Here $\mathrm{n}, \mathrm{nr}, \mathrm{q}$ are the number of the above variables or parameters respectively and $\mathrm{np}$ is the number of constraints. $f(*)$ is the objective function, $\mathrm{p}_{f \mathrm{i}}$ is a prescribed probability value. Here the superscript "N" in Equation 56 indicates the nominal value of each variables or parameters.

After a geometrical interpretation of the EBDO problems, a computationally efficient solution is presented and two design examples are provided to demonstrate the proposed EBDO method in Ref. (Mourelatos and Zhou, 2005). The algorithm quickly identifies the vicinity of the optimal point by a derivativefree optimizer calculating the evidence-based optimum, starting from the close-by RBDO optimum and moving a hyper-ellipse in the original design space, as used in RBDO algorithm. Moreover, only the identified active constraints are considered for local surrogate models. All these concerns keep the computational cost desirably low (Mourelatos and Zhou, 2005).

It is also shown that the EBDO is conservative compared with all RBDO designs obtained with different probability distributions. It provides the possibility to investigate design optimization in a more broadened and general point of view, if the uncertainty representation tools can be further improved.

\subsubsection{Other design Optimizations}

Robust design is developed to address the concern of robustness of certain performance parameters and reliability of the design (Du et al., 2003). In a robust design optimization, the variation in performance parameter can be either minimized, or constrained to be lower than some value. In a traditional robust optimization proposed by Taguchi, the main aim is to find designs with minimum variation of certain performance characteristics, i.e., to minimize the product quality loss. Using the property of orthogonal arrays, the robust design with minimum performance parameter variation can be identified.

A robust design method has been adopted in a variety of practical engineering problems. Recently there is a tendency of integrating it to uncertainty-based design optimizations with a result of enhancing product quality as well as confidence level (e.g., reliability) (Youn, 2005), although the emphasis of each individual paradigm is different.

The fuzzy set approach is common, where membership functions characterize the input uncertainty.

This method expresses uncertain input variables via lower and upper bounds and defines the output interval by the minimum and the maximum of all input end point combinations (Xiao et al., 2011).

It assumes that uncertain input variables lie within a convex region and be reduced to interval analysis when taking a hypercube convex region.

\subsubsection{Integration of Various Optimization Methods}

In recent years more and more attention of designers is paid to the integrated framework of uncertainty analysis and even more of design optimization methods, due to the modified design guideline or standard. Moreover, in order to deal with the situation when there is insufficient information, the possibilistic method itself or its integrated framework may be the better choice.

Such integration research includes integration of the probabilistic approach with the possibilistic approach (Youn et al., 2004; Langley, 2000), integration of the probabilistic approach with the evidential approach (Lee et al., 1987), integration of possibility-based design optimization with robust design (Youn et al., 2005; Huang et al., 2009), integration of probabilistic optimization with robust design (Du et al., 2003), integration of aleatory uncertainty with epistemic uncertainty for various design optimizations (Youn, 2005; Huang and Zhang, 2009) and so on. 


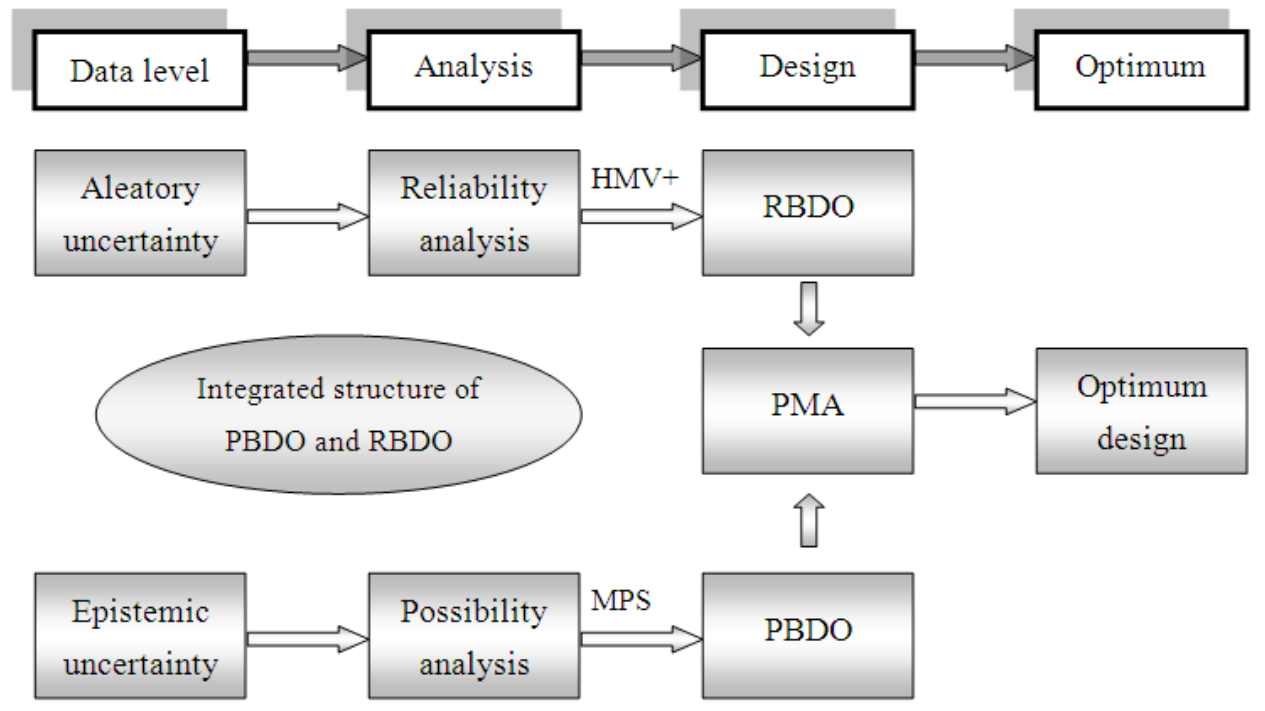

Fig. 8. An integration of PBDO and RBDO by PMA method (Youn et al., 2004)

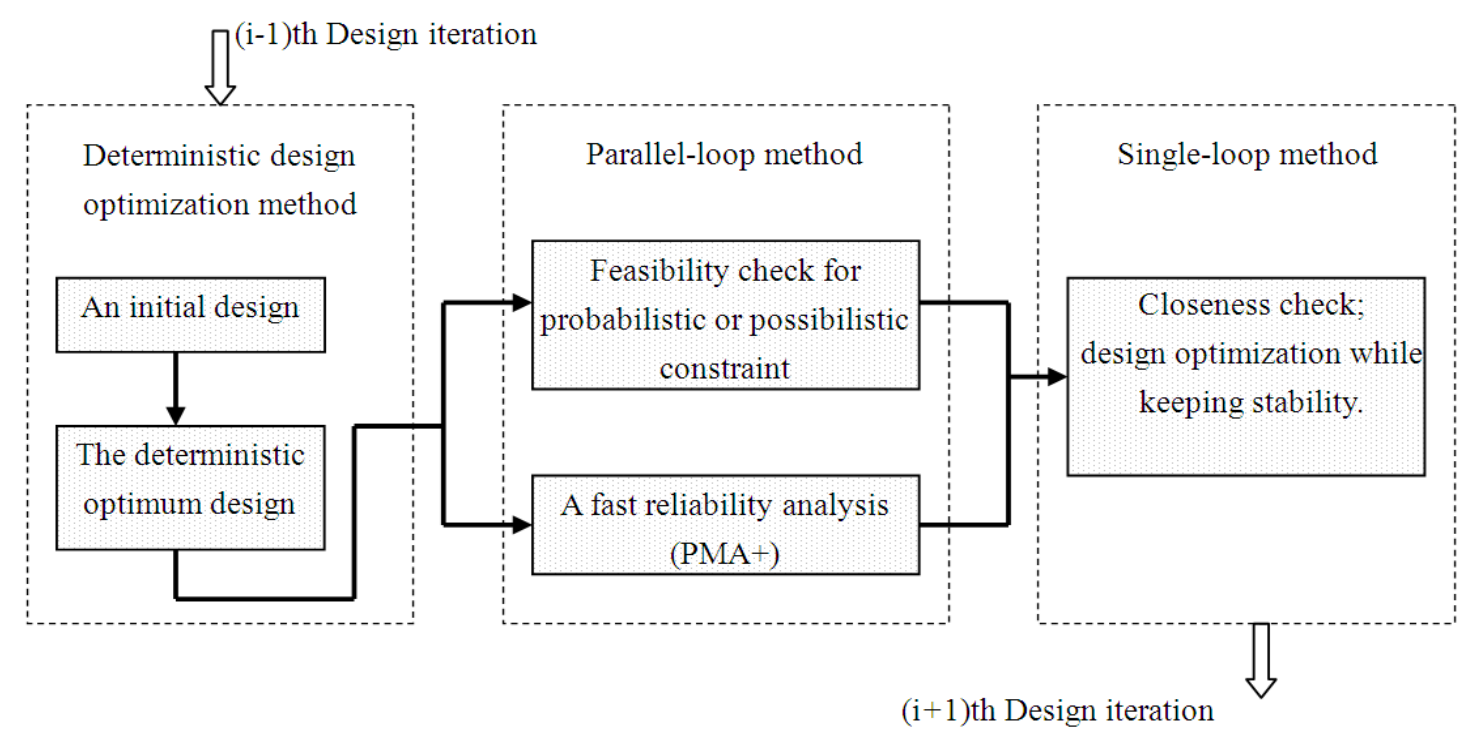

Fig. 9. The adaptive-loop method proposed by (Youn, 2005)

The survey of existing work is not all-inclusive, but rather representative work emphasizing those methods that can ultimately be expressed under a common analytical framework.

Youn and Choi (2004a) and Youn et al. (2004) present an integrated design platform of both RBDO and PBDO when modeling physical uncertainty with insufficient information, using PMA to improve numerical efficiency and stability in PBDO while MPS for highly nonlinear and monotonic performance response in RBDO. Such a structure is shown in Fig. 8.

In his study, the adaptive-loop method is composed of three phrases of optimization: The deterministic design optimization is employed at the beginning of the process with such additional improvement of numerical efficiency by reducing the design iterations and then the parallel-loop method is expedited addressing numerical convergence and statistical feasibility using PMA+, the 
last step is for single-loop method, checking the design closeness and improving computational efficiency. Such integrated framework is typical as an organic structure for uncertainty-design optimization. The adaptive-loop method is illustrated in Fig. 9.

In response to such improved standards in engineering design as reliability and robustness and considering the fact that, for epistemic uncertainties, a possibility-based design optimization deals with the failure rate, while a robust design optimization minimizes the product quality loss, researchers are interested in the integration work for epistemic uncertainty. Since there is no metric for product quality loss defined under epistemic uncertainty, (Youn et al., 2005) propose a new design framework successfully integrating the PBDO and a robust design optimization with new formulation of product quality loss for epistemic uncertainty. Such Possibility-Based Robust Design Optimization (PBRDO) can be formulated as Equation 57:

$$
\begin{array}{ll}
\min & \operatorname{Cost}(d, V) \\
\text { s.t. } & \Pi\left(G_{i}(d(V))>0\right) \leq \alpha_{t}, \quad i=1,2, \cdots, n p \\
& d^{L} \leq d \leq d^{U} \\
& d=m(V) \in R^{n d v} \text { and } \quad V=\left[V_{i}\right]^{T} \in R^{n r v}
\end{array}
$$

where, the design vector $d=m(V)$ is the maximum likely value of the fuzzy random vector and $\mathrm{V}$ is the fuzzy random vector and $n p$, ndv and nrv are the number of possibilistic constraints, the number of design variables and the number of fuzzy random variables, respectively.

First, their paper proposes a new metric for product quality loss in three different types of robust objectives. Then the MPS method and PMA+ are employed for more effectively estimating possibilistic constraints and conducting the design optimization, respectively. Two examples are used to show the feasibility of possibilitybased robust design with epistemic uncertainty, comparing with those results of reliability-based robust design optimization. Such framework is ready for application in other areas.

Actually, robust design can be integrated to any of uncertainty-based design optimizations with the result of enhancing product quality as well as confidence level (e.g., reliability).

Considering that robust design emphasizes on improving the product quality by minimizing the effects of variations while RBDO focuses on maintaining design feasibility at expected probabilistic levels, (Du et al.,
2003) propose an integrated framework of two methodologies for the design objective robustness with probabilistic constraints.

They employ an inverse reliability strategy that uses percentile performance and give a new search algorithm for the Most Probable Point of Inverse Reliability (MPPIR), evaluating the performance robustness and percentile performance in the proposed formulation.

Their engineering example of a vehicle combustion engine piston design illustrates the effectiveness of the method, solving the tradeoff problem encountered in the integration simultaneously which has ever been the difficulty in uncertainty handling.

\subsubsection{Data Fusion Technology in Risk and Reliability Analysis}

\subsubsection{A General Introduction of Information Fusion}

In the early $1980 \mathrm{~s}$, it was military scientists that were the pioneers in the use of techniques of what is now called information fusion (Hall and Llinas, 1997; Zhuang et al., 2000). Data fusion is now a formal framework and tools for the alliance of data originating from different sources of different nature. It aims at obtaining information of greater quality.

There are indeed a large amount of literatures relating to this method in different aspects of application areas, e.g., defense systems, geosciences, medicine and industrial engineering. The information involved in the fusion process may be data, image, sensor and classifier. So the concept of data fusion can be extended and the application area can also been extended.

A fusion system is usually multi-leveled, e.g., from fixed level, then to feature level and lastly to decision level. The mathematical tools used for fusion are various, including probability theory, evidence theory, fuzzy set and possibility theories and more recently neural networks (Hall and Llinas, 1997).

As two of the most important fusion methods, evidence method and possibility method have been widely used, including the area of reliability assessment and engineering design (Perrin et al., 2004).

\subsubsection{Information Fusion Using Possibility Based Method}

In the framework of possibility theory, the information available, relative to the value of parameter, is represented by a possibility distribution, which corresponds to an interval (or a set) representing imprecise information. Such a set is generally fuzzy. 
Hong-Zhong Huang et al. / American Journal of Engineering and Applied Sciences 6 (1): 95-136, 2013

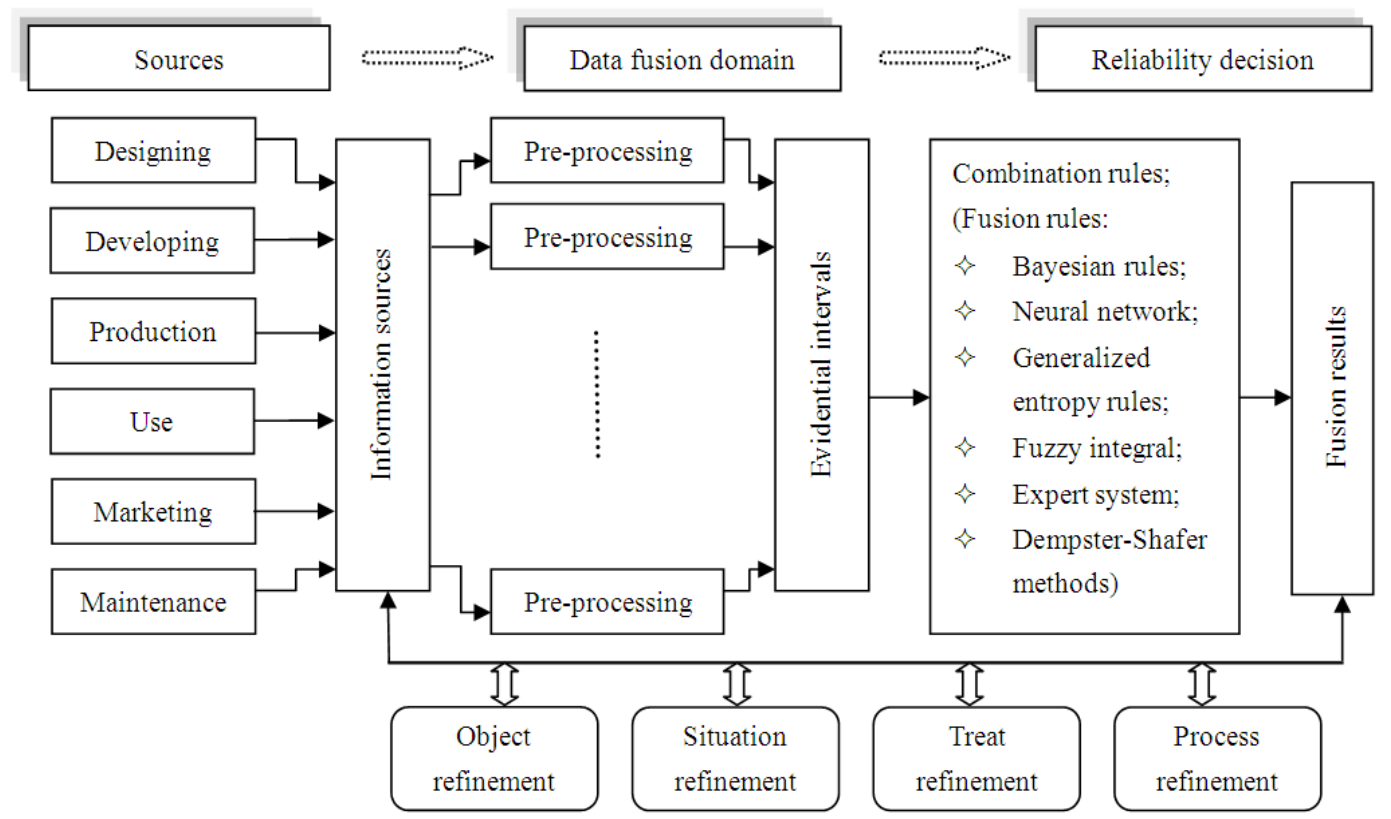

Fig. 10. Dempster-Shafer methods as a part of reliability information fusion model (Hall and Lawry, 2001)

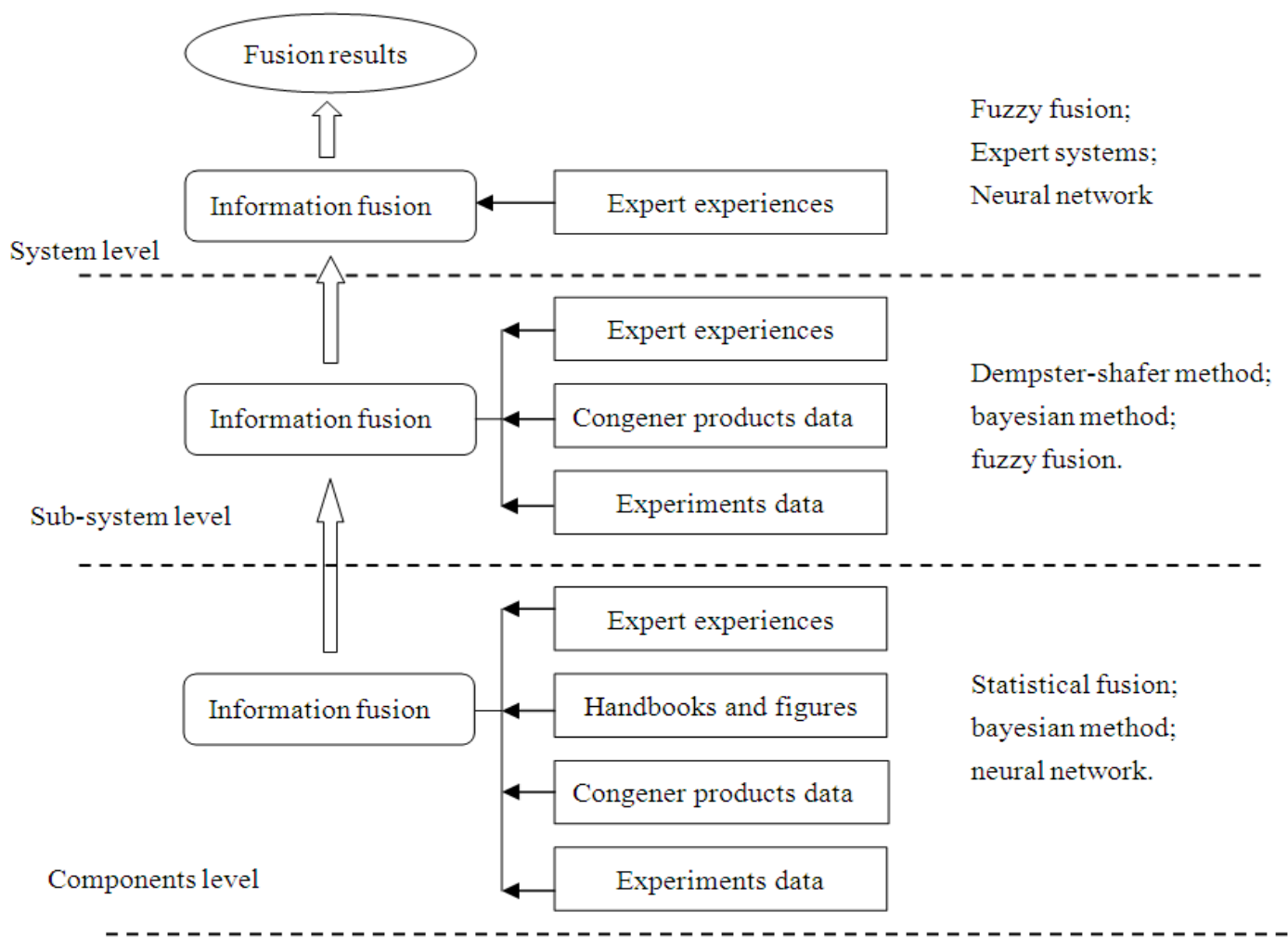

Fig. 11. An information fusion structure for comprehensive reliability assessment 
It is possible to find a large range of schemes suiting each case, where the fusion of uncertain information is equivalent to finding a compromise between a too accurate result which is certainly false and a sure result which is too imprecise.

Hence, the union and the intersection are both principal ways, among others, to fuse two sets, which provide a great opportunity for reliability analysis and evaluation.

\subsubsection{Information Fusion Using the DS Method}

As a generalization of the probability theory, the evidence theory allows the handling of non-exclusive and non-singleton events. Each measure attaches a probability to any element of the power set of the set of discernment (Hall and Llinas, 1997; Zhuang et al., 2000; Sentz and Ferson, 2002). The Dempster-Shafer rule is used to aggregate these input mass functions. Different modes of decision associated allow us to handle the compromise information. Based on this knowledge, with information theory, the DS fusion method in reliability engineering can be illustrated as Fig. 10.

Aiming at the difficulty in reliability assessment of complex large scale system, using information fusion technology is worth trying. The essential strategy should be considered as combining fusion technology into a comprehensive approach (Oberkampf et al., 2000). In the reliability assessment process, fusion technologies are first applied to subsystems, then synthesis all combine proper results together, as showed in Fig. 11 (Zhuang et al., 2000).

\subsection{Performability Improvement on the use of Possibility theory and Evidence Theory}

So far as we know, besides theoretical and computational developments by the means of possibilistic and evidential approaches, some physical problems have also been solved in the area of perfomability, which includes quality, reliability, maintenance and safety and risk. Such physical problems as failure mechanisms and detective methods are related to system failure engineering, which in some sense can be viewed as a part of operational research (Cai, 1996). From this point of view, fuzzy (possibilistic) methodology and evidence theory have made their own contributions to various aspects of dependability and performability. With fast advances in technology and the increasing complexity of technological systems, in a holistic point of view, product characteristics comprising functionality, reliability and maintainability have been becoming more and more important and alternative approaches are needed to address them.

Probability theory alone is not sufficient to solve the problem of human subjectivity as it does not follow the probability axioms. Possibility theory and DempsterShafer theory of evidence thus offer such alternative approaches by adoptions of natural language expressions about reliability information (Misra, 1993).

\subsubsection{Quality}

System quality, in a narrow sense as a body of performance indices whereas in a broad sense as all relevant variables and procedures, are often prone to human errors and management defects (Murthy and Djamaludin, 2002). Quality factors, even quality definitions are essentially fuzzy and unclear in reality. Applying fuzzy methodology, the inherent and intrinsic data and information can be represented in many examples including fuzzy control chart and fuzzy rules in quality control (Cai, 1996). The complex and fuzzy relationships among object system, operating situations and development process, supporting resources also call for fuzzy techniques.

\subsubsection{Reliability}

In the context of measure and integral, reliability is a quantitative index and can be measured by the opposite side, i.e., unreliable or failure events. The first adoption of fuzzy methodology in reliability and failure analysis, i.e., the proposed notion of component possibility as a reliability index, may be dated back to Kaufman's study (Kaufmann, 1983), although the motivation and exact meaning of component possibility were not explained at that time and now fuzzy-based approaches are appearing in various areas of reliability evaluation (Bai and Asgarpoor, 2004) and modeling (Delmotte and Borne, 1998).

Because of the simplicity of combination rules, it is shown recently that D-S theory has been used in various fields which were not common before, e.g., in system reliability settings, fault diagnosis (Fan and Zuo, 2006a; 2006b).

\subsubsection{Fault Tree Analysis}

Fault Tree Analysis (FTA) has been widely used as a powerful and efficient tool for reliability analysis and safety prediction. The visual and quantificational characteristics of FTA make it feasible in accordance with the trend of combining quantitative approaches with qualitative approaches, addressing limitations of the conventional probabilistic approach. Among others, we mention fuzzy reliability theories and fuzzy logic based method for linguistic (imprecise) quantification of fuzzy characteristics and construction of approximate reasoning system.

The first implementation of fuzzy method in the context of fault tree analysis was pioneered by 
(Tanaka et al., 1983), who treated imprecise probabilities of basic events as trapezoidal fuzzy numbers and employed the extension principle to describe the logical relationships leading to the top event. Furuta and Shiraishi (1984) also proposed a kind of importance measure but by means of max/min fuzzy operator and fuzzy integrals other than Tanaka's approach. When it comes to fuzzy number, (Singer, 1990) also thinks it as the perfectly straightforward way to overcome the deficiencies of inexact and inaccuracy knowledge. Soma et al. (1993) proposed a more general fuzzy method, also known as resolution identity to handle repeated events. Moreover they extended this method to deal with multi-state FTA (Misra and Soman, 1995). Another approach used to model imprecise relationships between physical and reliability states is proposed by (Pan and Yun, 1997), using fuzzy gates to describe output by triangular fuzzy numbers instead of crisp values 0 or 1 . In fact, by defining fuzzy possibility of fuzzy event analogous to fuzzy probability, FTA can take into consideration subjective and experts' opinion (Huang et al., 2004). Furthermore, fuzzy fault tree method has been implemented in engineering practice widely (Mentes and Helvacioglu, 2011; Jafarian and Rezvani, 2012; Al-Humaidi and Hadipriono, 2010).

Cai (1996) has summarized three main manners of fuzzy methodology in reliability engineering, respectively as:

- $\quad$ Treating the probability as a fuzzy number

- Defining reliability in terms of possibility measure

- Considering failure as a fuzzy event

\subsubsection{Failure Modes and Effects Analysis (FMEA)}

Failure Modes and Effects Analysis (FMEA) examines the failure cause-effect relationships. Both causes and effects can be fuzzy in some sense. Along with treating both probability and consequences of failures as fuzzy sets, interdependencies among various causes and effects may be assessed by the rule-based reasoning. Keller and Kara-Zaitri (1989) have observed this and introduced fuzzy logic to handle the impreciseness in fault representation. Gargama and Chaturvedi (2011) introduce fuzzy logic to describe the linguistic variables.

\subsubsection{Fault Diagnosis and Detection}

Fault diagnosis partially interprets the reasons why system fails. Fuzzy approach together with the idea of fuzzy logic and linguistic approach can be naturally used to deal with vagueness and ambiguity in system models and in human perceptions (Cai, 1996). Furthermore, failure detection and identification problems can be addressed by fuzzy logic and Dempster-Shafer theory (Misra, 1993), or along with probabilistic approaches in multi-source data analysis (Lee et al., 1987).

Since one of the central issues in the evidence theory is how to combine imperfect information given by independent knowledge sources, D-S theory of evidence has been gaining popularity in various fields when orienting to incomplete knowledge, e.g., multiple-fault diagnosis problem. Even though previous causal models for diagnostic expert systems are formulated in the framework of probability theory, the D-S theory of evidence has been suggested for solving some diagnostic problems.

Ishibuchi and his colleagues (Misra, 1993) developed such a diagnosis model to restrict their consideration to cases, where fuzzy symptoms are expressed by belief structures. Very recently (Fan and Zuo, 2006a; 2006b) have proposed new decision rules based on the improved D-S evidence theory and employed the improved method in gearbox fault diagnosis, which enhance diagnostic accuracy and autonomy by means of combining expert knowledge and multi-source information.

Even now, application of D-S evidence theory in diagnosis has just begun. Issues deserving study involve that how to transform expert diagnostic opinion into basic probability assignments and how to determine threshold precisely.

\subsubsection{Maintenance and Warranty}

When it comes to product failures, it is naturally mentioned the notions of maintenance and warranty. Maintenance involves actions to control the deterioration process leading to failure and actions to restore failed equipment to its operational states by corrective measures after a failure. A warranty is a contract between buyer and manufacturer to replace or repair a faulty item, or to partially or fully compensate the consumer in the case of failure.

Product maintenance and warranty have received the attention of researchers from many different disciplines and are related to sub-areas including optimal system design, optimal reliability improvement, modeling imperfect repairs and replacement.

To the best of our knowledge, the three formal views of warranty are the exploitation theory, the signal theory and the investment theory, respectively. On general grounds, the more reliable the product is, the lower the cost of replacement under warranty for the user. So warranty policies are structured according to perspectives of manufacture and buyer. There is a negative correlation between product quality and warranty costs. Murthy and Djamaludin (2002) and Wang et al. $(2009 ; 2010)$ for more details. 
The literature on warranties and preventive maintenance are vast. On the issue of improving the reliability of a product, one way is to eliminate infant mortality or initial failure rate with a burn-in program; another way is to upgrade the manufacturing process; and the third consideration may be outgoing inspection to eliminate nonconforming items (Misra, 1993). In these studies, new technologies and design methods may provide benefit to measurable improvement in quality and investment.

\subsubsection{Safety}

Concerning with a special kind of failure with catastrophic consequences, safety may be a part of reliability. Fuzzy methodology can be applied, although limited at present, in areas of safety design and safety assessment. Fuzzy rules can be adopted in expert judgments and subjective assessments.

If we must decide whether to operate or switch off a system based on available information which may be incomplete and imprecise, evidence theory can be explored to meet such demand. This is a kind of safety control problem and Dempster's rule of combination has been used for fusing a given set of information (Cai, 1996).

\subsubsection{Risk}

Risk is concerned with both failure consequences and failure occurrence uncertainty. Risk is also linked to decision-making, a policy and so on. Subjects about risks are divided into two phases: Risk assessments and risk management (Cai, 1996). When risk management is performed in relation to a Probabilistic Risk Assessment (PRA), the two activities are named as a Probabilistic Risk Assessment And Management (PRAM).

Quite a few research efforts have been made to establish a unified PRAM methodology where subjective assessment, value judgment, expertise and heuristics are being dealt with more objectively. However, to express the uncertainty of the event occurrence in terms of possibility measure, it is still an open and challenging problem how to define and assess risk of an event.

\section{DEVELOPING TRENDS OF POSSIBILITY AND EVIDENCE-BASED METHODS}

\subsection{The Possible Directions of Future Works}

Although significant progress has been made during the last two decades, the investigating and developing of possibility and evidence theory is still an active research domain. The probable and noticeable perspectives include:
- Integrating or perfecting already-existing integration methods

- In attempt to integrate possibilistic and probabilistic methods which have been proven to be efficient and matured, e.g., DS method with other related methods

○ Reducing design iteration and shorten searching interval using combination algorithms or genetic algorithm

- Enhancing computational accuracy and stability and numerical efficiency

- Focusing on those methods that can ultimately be expressed under a common analytical framework

○ Improving and solving the conflict problem of various uncertainties

- How to propagate uncertainty in a global angle

- Constructing an error-compensation feedback loop as a software improvement or an adaptive loop as a correction mechanism

- Uncertainty quantification analysis and risk assessment of precise systems or those which are difficult to measure.

- Design for six sigma as a new robust optimization formulation, incorporating approaches from structural reliability and robust design.

- Soft computing strategies as the cooperating framework with diverse methods

○ Basic cooperating with fuzzy logic, probabilistic reasoning and neural network

- More advanced cooperating with genetic algorithm, evidential reasoning, learning machine and chaos theory

- Combination of theoretical research and practical applications in real environment, from both the scientists and engineer's angle

- Design optimization methods under uncertainties for handling complex systems design with multiple failure modes and high nonlinear limit state functions

- Accuracy and efficient reliability analysis method with small probability of failure of systems under uncertainties

We strongly hope that reliability engineers will closely collaborate with statisticians in the development of models and methods, to ensure applications in a field where uncertainty often plays a significant role in decision making.

\subsection{Implication for Practice}

Uncertainties exist widely in practical engineering; these uncertainties which can be classified as epistemic 
or aleatory, come from limited data, incomplete information, ignorance and inherent variation. Parameters in systems usually can not be determined precisely due to these various uncertainties, especially for aerospace and some military industies, which often face the problem of insufficient data or imcomplete information. In these cases traditional probability-based reliability methods can not work well because they require that all probabilities or probability distributions be known and/or perfectly determined. Some recent research has pointed out that probability-based reliability methods are very sensitive to parameter values; a little variation in parameters will lead to large error calculations (Guo et al., 2001). This means that the results calculated by probability-based methods are unreliable in cases of limited data and incomplete information. Fortunately, possibility-and evidencetheories-based reliability methods have many advantages when compared with probability-based reliability methods. They are fit for situations in which data and information are limited or imcomplete, therefore, possibility- and evidence-theories-based reliability methods have become a focus for academic research and much has been achieved. Furthermore, these uncertainty methods are becoming increasingly popular for engineering practices such as those in the fields of aerospace, automobile engineering, nuclear energy and defense. This is because they help design safer and more reliable products under severe uncertainty than do traditional probability-based reliability methods.

\section{CONCLUSION}

In this study, we have given a detailed and annotated overview of possibility and evidence, both the fundamental theories and the application for reliability, risk and uncertainty analysis in engineering design when there is not sufficient input data available, due to specific uncertainty. From the comparison and relationship of the two measures, we conclude that possibility theory and evidence theory play a significant role in reliability analysis as well as design optimization considering various uncertainties, especially epistemic uncertainty from incomplete input data, due to their given representations and theoretic constructions. But there also leaves a relative space needing further exploration, for more general framework and performance characteristics in demand of new modified design criteria. Our holistic angle of view can provide a comprehensive understanding of existing approaches and future work.

\section{ACKNOWLEDGMENT}

This research was partially supported by the National Science Foundation of China under contract number 50775026.

\section{REFERENCES}

Agarwal, H., J.E. Renaud, E.L. Preston and D. Padmanabhan, 2004. Uncertainty quantification using evidence theory in multidisciplinary design optimization. Relia. Eng. Sys. Saf., 85: 281-294. DOI: $10.1016 /$ j.ress.2004.03.017

Al-Humaidi, H.M. and T. F. Hadipriono, 2010. A fuzzy logic approach to model delays in construction projects using rotational fuzzy fault tree models. Civ. Eng. Environ. Syst., 27: 329-351. DOI: 10.1080/10286600903150721

Aughenbaugh, J.M. and C.J.J Paredis, 2005. The value of using imprecise probabilities in engineering design. Proceedings of the Design Engineering Technical Conferences and Computers and Information in Engineering, Sep. 24-28, California, USA., pp: 469-481. DOI: 10.1115/DETC200585354

Augustin, T. and R. Hable, 2010. On the impact of robust statistics on imprecise probability models: A review. Struct. Saf., 32: 358-365. DOI: 10.1016/j.strusafe. 2010.06.002

Bae, H.R, R.V. Grandhia and R.A. Canfield, 2004a. An approximation approach for uncertainty quantification using evidence theory. Relia. Eng. Syst. Saf., 86: 215-225. DOI: 10.1016/j.ress.2004.01.011

Bae, H.R., R.V. Grandhi and R.A. Canfield, 2002. Uncertainty quantification of structural response using evidence theory. AIAA J., 41: 2062-2068.

Bae, H.R., R.V. Grandhi and R.A. Canfield, 2004b. Epistemic uncertainty quantification techniques including evidence theory for large-scale structures. Comput. Struct., 82: 1101-1112. DOI: 10.1016/j.compstruc.2004.03.014

Bai, X.G. and S. Asgarpoor, 2004. Fuzzy-based approaches to substation reliability evaluation. Electr. Pow. Syst. Res., 69: 197-204. DOI: 10.1016/j.epsr.2003.08.011

Beynon, M., B. Curry and P. Morgan, 2000. The Dempster-Shafer theory of evidence: An alternative approach to multicriteria decision modeling. Omega., 28: 37-50. DOI: 10.1016/S03050483(99)00033-X 
Bhattacharya, P., 2000. On the Dempster-Shafer evidence theory and non-hierarchical aggregation of belief structures. IEEE. Trans. Syst. Man. Cyb., 30: 526-536. DOI: 10.1109/3468.867860

Borotschnig, H., L. Paletta and M. Prantl, 1999. A comparison of probabilistic, possibilistic and evidence theoretic fusion schemes for active object recognition. Comput., 62: 293-319. DOI: 10.1007/s006070050026

Buckley, J.J., 1988. Possibility and necessity in optimization. Fuzzy Set. Syst., 25: 1-13. DOI: 10.1016/0165-0114(88)90094-2

Cai, K.Y., 1996. System failure engineering and fuzzy methodology: An introductory overview. Fuzzy Set. Syst., 83: 113-133. DOI: 10.1016/01650114(95)00385-1

Cai, K.Y., C.Y. Wen and M.L. Zhang, 1991b. Posbist reliability behavior of typical systems with two types of failure. Fuzzy Set. Syst., 43: 17-32. DOI: 10.1016/0165-0114(91)90018-L

Cai, K.Y., C.Y. Wen and M.L. Zhang, 1993. Fuzzy states as a basis for a theory of fuzzy reliability. Microelect. Reliab., 33: 2253-2263. DOI: 10.1016/0026-2714(93)90065-7

Cai, K.Y., C.Y. Wen and M.L. Zhang, 1995a. Mixture models in profust reliability theory. Microelect. Reliab., 35: 985-993. DOI: 10.1016/00262714(94)00058-V

Cai, K.Y., C.Y. Wen and M.L. Zhang, 1995b. Posbist reliability behavior of fault-tolerant systems. Microelect. Reliab., 35: 49-56. DOI: 10.1016/00262714(94)00052-P

Cai, K.Y., C.Y. Wen and W.L. Zhang, 1991a. Fuzzy variables as a basis for a theory of fuzzy reliability in the possibility context. Fuzzy Set. Syst., 42: 145172. DOI: 10.1016/0165-0114(91)90143-E

Cappelle, B. and E.E. Kerre, 1993. On a possibilistic approach to reliability theory. Proceedings of the 2nd International Symposium on Uncertainty Analysis, Apr. 25-28, IEEE Xplore Press, College Park, MD., pp: 415-418. DOI: 10.1109/ISUMA.1993.366736

Cappelle, B. and E.E. Kerre, 1994. A general possibilistic framework for reliability theory. IPMU, 945: 311-317. DOI: 10.1007/BFb0035964

Cappelle, B. and E.E. Kerre, 1995a. An algorithm to compute possibilistic reliability. Proceedings of the 3rd International Symposium on Uncertainty Modeling and Analysis, Sep. 17-19, IEEE Xplore Press, College Park, MD., pp: 350-354. DOI: 10.1109/ISUMA.1995.527720
Cappelle, B. and E.E. Kerre, 1995b. Computer assisted reliability analysis: An application of possibilistic reliability theory to a subsystem of a nuclear power plant. Fuzzy Set. Syst., 74: 103-113. DOI: 10.1016/0165-0114(95)00040-R

Cayrac, D., D. Dubois and H. Prade, 1996. Handling uncertainty with possibility theory and fuzzy sets in a satellite fault diagnosis application. IEEE Trans. Fuzzy Syst., 4: 251-269. DOI: 10.1109/91.531769

Chen, S., E. Nikolaidis, H.H. Cudney, R. Rosca and R.T. Haftka, 1999. Comparison of probabilistic and fuzzy set methods for designing under uncertainty. American Institute of Aeronautics and Astronautics.

Choi, K.K., L. Du and B.D. Youn, 2004. A new fuzzy analysis method for possibility-based design optimization. Proceedigns of the 10th AIAA/ISSMO Symposium on Multidisciplinary Analysis and Optimization, (ASMAO' 04), University of Iowa, pp: 1-1. DOI: $10.2514 / 6.2004-4585$

Coolen, F.P.A., 2004. On the use of imprecise probabilities in reliability. Q. Reliab. Eng. Int., 20: 193-202. DOI: 10.1002/qre.560

Coolen, F.P.A., P. Coolen-Schrijner and K.J. Yan, 2002. Nonparametric predictive inference in reliability. Relia. Eng. Sys. Saf., 78: 185-193. DOI: 10.1016/S0951-8320(02)00162-X

De-Cooman, G., 1997. Possibility theory-I, II, III. Int. J. Gen. Syst., 25: 291-371.

Delgado, M. and S. Moral, 1987. On the concept of possibility-probability consistency. Fuzzy Set. Syst., 21: 311-318. DOI: 10.1016/0165-0114(87)90132-1

Delmotte, F. and P. Borne, 1998. Modeling of reliability with possibility theory. IEEE. Trans. Syst. Man. Cyb., 20: 78-88. DOI: 10.1109/3468.650324

Dempster, A.P., 1967. Upper and lower probabilities induced by a multivalued mapping. Ann. Math. Stat., 38: 325-339. DOI: 10.1214/aoms/1177698950

Dhanesh, P., 2003. Reliability-based optimization for multidisciplinary system design. Ph.D. Thesis, University of Notre Dame.

Drakopoulos, J.A., 1995. Probabilities, possibilities and fuzzy sets. Fuzzy Set. Syst., 75: 1-15. DOI: 10.1016/0165-0114(94)00341-4

Du, X. and W. Chen, 2004. Sequential optimization and reliability assessment for probabilistic design. J. Mech. Design., 126: 225-233. DOI: $10.1115 / 1.1649968$

Du, X., A. Sudjianto and W. Chen, 2003. An integrated framework for probabilistic optimization using inverse reliability strategy. Proceedings of Design Engineering Technical Conferences and Computers and Information in Engineering Conference, Sep. 26, Chicago, Illinois, USA., pp: 25-34. DOI: 10.1115/DETC2003/DAC-48706 
Dubois, D. and H. Prade, 1980. Fuzzy Sets and Systems: Theory and Applications. 1st Edn., Academic Press, New York, ISBN-10: 9780080917726, pp: 393.

Dubois, D. and H. Prade, 1983. Unfair coins and necessity measure: Towards a possibilistic interpretation of histograms. Fuzzy Set. Syst., 10: 15-20. DOI: 10.1016/S0165-0114(83)80099-2

Dubois, D. and H. Prade, 1987. An alternative approach to the handling of subnormal possibility distributions- A critical comment on a proposal by Yager. Fuzzy Set. Syst., 24: 123-126. DOI: 10.1016/0165-0114(87)90122-9

Dubois, D. and H. Prade, 1988. Possibility Theory: An Approach to Computerized Processing of Uncertainty. Plenum Press, New York, ISBN-10: 0306425203, pp: 263.

Dubois, D., H. Prade and S. Sandri, 1993. On Possibiiity/Pmbability Transformations. In: Fuzzy Logic: State of the Art, Lowen, R. and M.R. Roubens (Eds.), Kluwer Academic Publication, New York, ISBN-10: 0792323246.

Fan, X.F. and M.J. Zuo, 2006a. Fault diagnosis of machines based on D-S evidence theory. Part 1: D-S evidence theory and its improvement. Patt. Recogn. Lett., 27: 366-376. 10.1016/j.patrec.2005.08.025

Fan, X.F. and M.J. Zuo, 2006b. Fault diagnosis of machines based on D-S evidence theory. Part 2: Application of the improved D-S evidence theory in gearbox fault diagnosis. Patt. Recogn. Lett., 27: 377 385. DOI: 10.1016/j.patrec.2005.08.024

Ferson, S. and L.R. Ginzburg, 1996. Different methods are needed to propagate ignorance and variability. Reliab. Eng. Syst. Saf., 54: 133-144. DOI: 10.1016/S0951-8320(96)00071-3

Fioretti, G., 2004. Evidence theory: A mathematical framework for unpredictable hypotheses. Metroecnomica, 55: 345-366. DOI: 10.1111/j.1467999X.2004.00197.x

Florea, M. C., A.L. Jousselme, E. Bosse and D. Grenier, 2009. Robust combination rules for evidence theory. Inform. Fusion., 10: 183-197. DOI: 10.1016/j.inffus.2008.08.007

Furuta, H. and N. Shiraishi, 1984. Fuzzy importance in fault tree analysis. Fuzzy Set. Syst., 12: 205-214. DOI: 10.1016/0165-0114(84)90068-X

Gaines, B.R. and L.J. Kohout, 1975. The logic of automata. Int. J. General Syst., 2: 191-208. DOI: 10.1080/03081077608547469

Gargama, H. and S.K. Chaturvedi, 2011. Criticality assessment models for failure mode effects and criticality analysis using fuzzy logic. IEEE Trans. Reliab., 60: 102-110. DOI: 10.1109/TR.2010.2103672
Giles, R., 1982. Foundations for a Theory of Possibility, Fuzzy Information and Decision Processes. 1st Edn., North-Holland Publ. Co., Amsterdam, ISBN-10: 0444864911, pp: 451.

Greene, M.S., Y. Liu and W. Chen, 2011. Computational uncertainty analysis in multiresolution materials via stochastic constitutive theory. Comput. Method. Applied Mech. Eng., 200: 309-325. DOI: 10.1016/j.cma.2010.08.013

Guo, S.X., Z.Z. Lu and Y.S. Feng, 2001. A nonprobabilistic model of structural reliability based on interval analysis. Chin. J. Computat. Mech., 18: 56-60.

Hall, D.L. and J. Llinas, 1997. An introduction to multisensor data fusion. Proc. IEEE, 85: 6-23. DOI: $10.1109 / 5.554205$

Hall, J. and J. Lawry, 2001. Imprecise probabilities of engineering system failure from random and fuzzy set reliability analysis. Proceedings of the 2nd International Symposium on Imprecise Probabilities and Their Applications, Jun. 26-29, Cornell University, Ithaca, New York.

Hegarat-Mascle, L.S., I. Bloch and D. Vidal-Madjar, 1997. Application of Dempster-Shafer evidence theory to unsupervised classification in multisource remote sensing. IEEE. Trans. Geosci. Remote., 35: 1018-1031. DOI: $10.1109 / 36.602544$

Henkind, S.J. and M.C. Harrison, 1988. An analysis of four uncertainty calculi. IEEE. Trans. Syst. Man. Cyb., 18: 700-714. DOI: 10.1109/21.21598

Huang H.Z., X. Zhang, W. Yuan, D. Meng and X. Zhang, 2011b. Collaborative reliability analysis under the environment of multidisciplinary design optimization. Concurrent Eng. Res. A., 19: 245-254. DOI: $10.1177 / 1063293 X 11420177$

Huang, H.Z, 2012. Structural reliability analysis using fuzzy sets theory. Eksploatacja NiezawodnoscMaintenance Reliab., 14: 284-295.

Huang, H.Z. and X. Zhang, 2009. Design optimization with discrete and continuous variables of aleatory and epistemic uncertainties. J. Mech. Design, Trans. ASME, 131: 031006-031013. DOI: $10.1115 / 1.3066712$

Huang, H.Z., 1995. Reliability analysis method in the presence of fuzziness attached to operating time. Microelect. Reliab., 35: 1483-1487. DOI: 10.1016/0026-2714(94)00173-L

Huang, H.Z., 1996. Reliability evaluation of a hydraulic truck crane using field data with fuzziness. Microelect. Reliab., 36: 1531-1536. DOI: 10.1016/0026-2714(95)00213-8 
Huang, H.Z., 1997. Fuzzy multi-objective optimization decision-making of reliability of series system. Microelect. Reliab., 37: 447-449. DOI: 10.1016/S0026-2714(96)00040-6

Huang, H.Z., H. Xu and L. He, 2009. GA based fuzzy multi-objective robust design. J. Mult. Valued Log. Soft. Comput., 15: 39-50.

Huang, H.Z., M.J. Zuo and Z.Q. Sun, 2006d. Bayesian reliability analysis for fuzzy lifetime data. Fuzzy Set. Syst., 157: 1674-1686. DOI: 10.1016/j.fss.2005.11.009

Huang, H.Z., P. Wang, M.J. Zuo, W.D. Wu and C.S. Liu, 2006c. A fuzzy set based solution method for multiobjective optimal design problem of mechanical and structural systems using functionallink net. Neural Comput. Appl., 15: 239-244. DOI: 10.1007/s00521-006-0025-2

Huang, H.Z., R.F. Bo and W. Chen, 2006a. An integrated computational intelligence approach to product concept generation and evaluation. Mech. Mach. Theory., 41: 567-583. DOI: 10.1016/j.mechmachtheory.2005.07.006

Huang, H.Z., W.D. Wu and C.S. Liu, 2005b. A coordination method for fuzzy multi-objective optimization of system reliability. J. Intell. Fuzzy Syst., 16: 213-220.

Huang, H.Z., X. Tong and M.J. Zuo, 2004. Posbist fault tree analysis of coherent systems. Reliab. Eng. Syst. Saf., 84: 141-148. DOI: 10.1016/j.ress.2003.11.002

Huang, H.Z., X. Zhang, D.B. Meng, Y. Liu and Y.F. Li, 2012a. A new multidisciplinary design optimization method accounting for discrete and continuous variables under aleatory and epistemic uncertainties. Int. J. Comput. Intell. Syst., 5: 93-110. DOI: 10.1080/18756891.2012.670524

Huang, H.Z., X. Zhang, Z. Wang, D.B. Meng and Y. Liu, 2012b. Enhanced sequential optimization and reliability assessment for reliability-based design optimization. J. Mech. Sci. Technol., 26: 2039-2043. DOI: $10.1007 / \mathrm{s} 12206-012-0511-7$

Huang, H.Z., Y. Li and Y. Liu, 2010. Posbist reliability theory of k-out-of-n: G System. J. Mult. Valued Log. Soft Comput., 16: 45-63.

Huang, H.Z., Y. Tao and Y. Liu, 2008. Multidisciplinary collaborative optimization using fuzzy satisfaction degree and fuzzy sufficiency degree model. Soft Comput., 12: 995-1005. DOI: 10.1007/s00500-0070268-6

Huang, H.Z., Y.K. Gu and X.P. Du, 2006b. An interactive fuzzy multi-objective optimization method for engineering design. Eng. Applied Artif. Intel., $\quad$ 19: $451-460 . \quad$ DOI: 10.1016/j.engappai.2005.12.001
Huang, H.Z., Z.G. Tian and M.J. Zuo, 2005a. Intelligent interactive multi-objective optimization method and its application to reliability optimization. IIE Trans., 37: 983-993. DOI: 10.1080/07408170500232040

Huang, H.Z., Z.L. Wang, Y.F. Li, B. Huang, N.C. Xiao and L.P. He, 2011a. A nonprobabilistic set model of structural reliability based on satisfaction degree of interval. Mechanika.

Jafarian, E. and M. Rezvani, 2012. Application of fuzzy fault tree analysis for evaluation of railway safety risks: An evaluation of root causes for passenger train derailment. J. Railb. Rap. Trans., 226: 14-25. DOI: $10.1177 / 0954409711403678$

Jumarie, G., 1994. Possibility-possibility transformation: A new result via information theory of deterministic functions. Kybernetes, 23: 54-59. DOI: 10.1108/03684929410064509

Kangas, A.S. and J. Kangas, 2004. Probability, possibility and evidence: Approaches to consider risk and uncertainty in forestry decision analysis. Forest. Policy Econ., 6: 169-188. DOI: 10.1016/S1389-9341(02)00083-7

Kaufmann, A., 1983. Advances in Fuzzy Sets: An Overview. In: Advances in Fuzzy Sets, Possibility Theory and Applications, Wang, P.P. (Ed.), Plenum Press, New York, ISBN-10: 0306413906.

Keller, A.Z. and C. Kara-Zaitri, 1989. Further applications of fuzzy logic to reliability assessment and safety analysis. Microelect. Reliab., 29: 399404. DOI: 10.1016/0026-2714(89)90627-6

Klir, G.J. and B. Parviz, 1992. Probability-possibility transformations: A comparison. Int. J. Gen. Syst., 21: 291-310. DOI: 10.1080/03081079208945083

Klir, G.J. and B. Yuan, 1995. Fuzzy Sets and Fuzzy Logic: Theory and Applications. 1st Edn., PrenticeHall, New York, ISBN-10: 0131011715, pp: 574.

Klir, G.J. and T.A. Folger, 1988. Fuzzy Sets, Uncertainty and Information. 1st Edn., Prentice Hall, New Jersey, ISBN-10: 0133459845, pp: 355.

Klir, G.J., 1995. Principles of uncertainty: What are they? Why do we need them? Fuzzy Set. Syst., 74: 15-31. DOI: 10.1016/0165-0114(95)00032-G

Klir, G.J., 2000. Fuzzy Sets: An Overview of Fundamentals, Applications and Personal Views. 1st Edn., Beijing Normal University Press, Beijing, ISBN-10: 7303052941, pp: 358.

Kohlas, J. and P.A. Monney, 1994. Theory of evidenceA survey of its mathematical foundations, applications and computational aspects. Math. Meth. Operat. Res., 39: 35-68. DOI: 10.1007/BF01440734 
Kozine, I.O. and Y.V. Filimonov, 2000. Imprecise reliabilities: Experiences and advances. Reliab. Eng. Sys. Saf., 67: 75-83. DOI: 10.1016/S09518320(99)00044-7

Langley, R.S., 2000. Unified approach to probabilistic and possibilistic analysis of uncertain systems. J. Eng. Mech., 126: 1163-1172. DOI: 10.1061/(ASCE)0733-9399(2000)126:11(1163)

Lee, T., J.A. Richards and P.H. Swain, 1987. Probabilistic and evidential approaches for multisource data analysis. IEEE. Trans. Geosci. Remote, $\quad$ GE-25: 283-293. DOI: 10.1109/TGRS.1987.289800

Li, Y. F., H.Z. Huang, Y. Liu, N. Xiao and H. Li, 2012. A new fault tree analysis method: Fuzzy dynamic fault tree analysis. Eksploat. Niezawodn., 14: 208214.

Liang, J.H., Z.P. Mourelatos and J. Tu, 2008. A singleloop method for reliability-based design optimisation. Int. J. Proje., 5: 76-92. DOI: 10.1504/IJPD.2008.016371

Limbourg, P., 2005. Multi-objective optimization of problems with epistemic uncertainty. Proceedings of the 3rd International Conference on Evolutionary Multi-Criterion Optimization, (EMO’ 05), SpringerVerlag Berlin, Heidelberg, pp: 413-427. DOI: 10.1007/978-3-540-31880-4 29

Liu, W.K., L. Siad, R. Tian, S.H. Lee and D. Lee et al., 2009. Complexity science of multiscale materials via stochastic computations. Int. J. Numer. Meth. Eng., 80: 932-978. DOI: 10.1002/nme.2578

Liu, Y. and H.Z. Huang, 2010. Reliability assessment for fuzzy multi-state systems. Int. J. Syst. Sci., 41: 365379. DOI: $10.1080 / 00207720903042939$

Liu, Y. and H.Z. Huang, 2011. Optimal replacement policy for fuzzy multi-state element. J. Mult-Valued Log. Soft Comput., 17: 69-92. DOI: 10.1109/TR.2010.2051242

Liu, Y., H.Z. Huang and G. Levitin, 2008. Reliability and performance assessment for fuzzy multi-state element. Proc. Institution Mech. Eng., Part O, J. Risk Reliab., 222: 675-686. DOI: 10.1243/1748006XJRR180

Liu, Y., M.S. Greene, W. Chen and D.A. Dikin, 2012. Computational microstructure characterization and reconstruction for stochastic multiscale material design. Comput. Aided. Design, 45: 65-76. DOI: 10.1016/j.cad.2012.03.007

Lowrance, J., T. Garvey and T. Strat, 1986. A framework for evidential reasoning systems. Proceedings of the 5th National Conference of American Association in Artificial Intelligence, (AAAI’ 86), pp: 896-903.
Mentes, A. and I.S. Helvacioglu, 2011. An application of fuzzy fault tree analysis for spread mooring systems. Ocean. Eng., 38: 285-294. DOI: 10.1016/j.oceaneng.2010.11.003

Misra, K.B. and K.P. Soman, 1995. Multistate fault Tree Analysis using Fuzzy Probability Vectors and Resolution Identity, In: Reliability and Safety Analyses Under Fuzziness, Onisawa, T. and Kacprzyk, J. (Eds.), Physica-Verlag, Heidelberg, ISBN-10: 3790808377, pp: 113-125.

Misra, K.B., 1993. New Trends in System Reliability Evaluation. 1st Edn., Elsevier Press, New York, ISBN-10: 0444816607, pp: 715.

Moens, D. and D. Vandepitte, 2004. Non-probabilistic approaches for non-deterministic dynamic FE analysis of imprecisely defined structures. Uncertainties in Structural Dynamics and Acoustics, pp: 3095-3120.

Moller, B., M. Beer, W. Graf and A. Hoffmann, 1999. Possibility theory based safety assessment. Comput. Aided. Civ. Inform., 14: 81-91. DOI: 10.1111/08859507.00132

Moller, B., W. Graf and M. Beer, 2004. Discussion on "Structural reliability analysis through fuzzy number approach, with application to stability". Comput. Struct., 82: 325-327. DOI: 10.1016/S00457949(03)00336-5

Moller, B., W. Graf, M. Beer and J. Sickert, 2006. Timedependent reliability of textile-strengthened $\mathrm{RC}$ structures under consideration of fuzzy randomness. Comput. Struct., 84: 585-603. DOI: 10.1016/j.compstruc.2005.10.006

Mourelatos, Z. and J. Zhou, 2004. Reliability estimation and design with insufficient data based on possibility theory. AIAA J., 43: 1696-1705. DOI: 10.2514/1.12044

Mourelatos, Z.P. and J. Zhou, 2005. A design optimization method using evidence theory. J. Mech. Des., 128: 901-908. DOI: 10.1115/1.2204970

Murthy, D.N.P. and I. Djamaludin, 2002. New product warranty: A literature review. Int. J. Prod. Econ., 79: 231-260. DOI: 10.1016/S0925-5273(02)00153-6

Nikolaidis, E. and R.T. Haftka, 2001. Theories of uncertainty for risk assessment when data is scarce.

Nikolaidis, E., S. Chen and H.H. Cudney, 2004. Comparison of probability and possibility for design against catastrophic failure under uncertainty. J. Mech. Des., 126: 386-394. DOI: 10.1115/1.1701878

Oberguggenberger, M, J. King and B. Schmelzer, 2009. Classical and imprecise probability methods for sensitivity analysis in engineering: A case study. Int. J. Approx. Reason., 50: 680-693. DOI: 10.1016/j.ijar.2008.09.004 
Oberkampf W.L., J.C. Helton, C.A. Joslyn, S.F. Wojtkiewicz and S. Ferson, 2004. Challenge problems: uncertainty in system response given uncertain parameters. Reliab. Eng. Sys. Saf., 85: 1119. DOI: $10.1016 /$ j.ress.2004.03.002

Oberkampf, W.L., S.M. DeLand, B.M. Rutherford, K.V. Diegert and K.F. Alvin, 2000. Estimation of total uncertainty in modeling and simulation.

Onisawa, T., 1988. An approach of system reliability analysis using failure possibility and success possibility. Fuzzy Set. Syst., 27: 87-103. DOI: 10.1109/FUZZY.1995.409963

Pan, H.S and W.Y. Yun, 1997. Fault tree analysis with fuzzy gates. Comput. Ind. Eng., 33: 569-572. DOI: 10.1016/S0360-8352(97)00195-2

Pang, Y., H.Z. Huang, N.C. Xiao, Y. Liu and Y.F. Li, 2012. Possibilistic reliability analysis of repairable system with omitted or delayed failure effects. Eksploat. Niezawodn., 14: 195-202.

Papalambros, P.Y. and N.F. Michelena, 2000. Trend and challenges in system design optimization. Proceedings of the International Workshop on Multidisciplinary Design Optimization, Aug. 7-10, Pretoria, S. Africa.

Perrin, S., E. Duflos and P. Vanheeghe, 2004. Multisensor fusion in the frame of evidence theory for landmines detection. IEEE Trans. Syst., Man, Cybern., 34: 485-498. 10.1109/TSMCC.2004.829266

Robinson, D.G., 1998. A survey of probabilistic methods used in reliability, risk and uncertainty analysis: Analytical techniques I. Sandia National Laboratories report.

Rowe, W.D., 1994. Understanding uncertainty. Risk Ana., 14: 743-750. DOI: 10.1111/j.15396924.1994.tb00284.x

Sentz, K. and S. Ferson, 2002. Combination of evidence in dempster-shafer theory. Department of Energy, Sandia National Laboratories, U.S.

Shafer, G., 1976. A Mathematical Theory of Evidence. 1st Edn., Princeton University Press, New Jersey, ISBN-10: 0691081751, pp: 297.

Singer, D., 1990. A fuzzy set approach to fault tree and reliability analysis. Fuzzy Set. Syst., 34: 145-155. DOI: 10.1016/0165-0114(90)90154-X

Soma, M.R., E. Donetti, C. Parolini, G. Mazzini and C. Ferrari et al., 1993. HMG CoA reductase inhibitors. In vivo effects on carotid intimal thickening in normocholesterolemic rabbits. Arteriosclerosis, Thromb. Vascular Biol., 13: 571-578 DOI: 10.1161/ 01.ATV.13.4.571
Soundappan, P., E. Nikolaidis, R.T. Haftka, R. Grandhi and R. Canfiedl, 2004. Comparison of evidence theory and Bayesian theory for uncertainty modeling. Reliab. Eng. Syst. Saf., 85: 295-311. DOI: $10.1016 /$ j.ress.2004.03.018

$\mathrm{Su}$, J. and J.E. Renaud, 1997. Automatic differentiation in robust optimization. AIAA J., 5: 1072-1079. DOI: $10.2514 / 2.196$

Sudkamp, T., 1992. The semantics of plausibility and possibility. Int. J. Gen. Syst., 21: 273-289. DOI: 10.1080/03081079208945082

Sun, R., H.Z. Huang and Q. Miao, 2008. Improved information fusion approach based on D-S evidence theory. J. Mech. Sci. Technol., 22: 2417-2425. DOI: 10.1007/s12206-008-0801-2

Tanaka, H, L.T. Fan, F.S. Lai and K. Toguchi, 1983. Fault-tree analysis by fuzzy probability. IEEE Trans. Reliab., 32: 453-457. DOI: 10.1109/TR.1983.5221727

Tu, J. and K.K. Choi, 1999. A new study on reliabilitybased design optimization. J. Mech. Des., 121: 557564. DOI: 10.1115/1.2829499

Utkin, L.V. and F.P.A. Coolen, 2007. Imprecise reliability: An introductory overview. Comput. Intell. Reliab. Eng., 40: 261-306. DOI: 10.1007/9783-540-37372-8 10

Utkin, L.V. and S.V. Gurov, 1996. A general formal approach for fuzzy reliability analysis in the possibility context. Fuzzy Set Syst., 83: 203-213. DOI: 10.1016/0165-0114(95)00391-6

Utkin, L.V., 1994. Fuzzy reliability of repairable systems in the possibility context. Microelect. Reliab., 34: 1865-1876. DOI: 10.1016/0026-2714(94)90282-8

Utkin, L.V., S.V. Gurov and I.B. Shubinsky, 1995. A method to solve fuzzy reliability optimization problem. Microelect. Reliab., 35: 171-181. DOI: 10.1016/0026-2714(95)90083-3

Walley, P., 1991. Statistical Reasoning with Imprecise Probabilities. 1st Edn., Chapman and Hall, London, ISBN-10: 0412286602.

Wang, P., 1994. A defect in Dempster-Shafer theory. Uncertainty of Artificial Intelligence.

Wang, Z., H.Z. Huang and L. Du, 2011. Reliability analysis on competitive failure under fuzzy degradation data. Applied Soft. Comput., 11: 29642973. DOI: $10.1016 /$ j.asoc.2010.11.018

Wang, Z., H.Z. Huang and X. Du, 2009. Reliabilitybased design incorporating several maintenance policies. Eksploat. Niezawodn., 4: 37-44.

Wang, Z., H.Z. Huang and X. Du, 2010. Optimal design accounting for reliability, maintenance and warranty. J. Mech. Des., 132: 011007.1-011007.8. DOI: $10.1115 / 1.4000638$ 
Wang, Z., H.Z. Huang, Y. Li, Y. Pang and N.C. Xiao, 2012. An approach to system reliability analysis with fuzzy random variables. Mech. Mach. Theory, 52: 35-46. DOI: 10.1016/j.mechmachtheory.2012.01.007

Wonneberger, S., 1994. Generalization of an invertible mapping between probability and possibility. Fuzzy Set Syst., 64: 229-240. DOI: 10.1016/01650114(94)90336-0

Wu, H.D, M. Siegel, R. Stiefelhagen and J. Yang, 2002. Sensor fusion using Dempster-Shafer theory [for context-aware HCI]. Proceedings of the 19th IEEE Instrumentation and Measurement Technology Conference May 21-23, IEEE Xplore Press, pp: $7-$ 12. DOI: 10.1109/IMTC.2002.1006807

Wu, J.S., G.E. Apostolakis and D. Okrent, 1990. Uncertainties in system analysis: Probabilistic versus non-probabilistic theories. Reliab. Eng. Syst. Saf., 30: 163-181. DOI: 10.1016/09518320(90)90093-3

Wu, W.Z., 2008. Attribute reduction based on evidence theory in incomplete decision systems. Inform. Sci., 178: 1355-1371. DOI: 10.1016/j.ins.2007.10.006

Xiao, N.C., H.Z. Huang, Z. Wang, Y. Li and Y. Liu, 2012. Reliability analysis of series systems with multiple failure modes under epistemic and aleatory uncertainties. Proc. Inst. Mech. Eng. O. J. Risk Reliab., 226: 295-304. DOI: $10.1177 / 1748006$ X11421266

Xiao, N.C., H.Z. Huang, Z. Wang, Y. Pang and L. He, 2011. Reliability sensitivity analysis for structural systems in interval probability form. Struct. Multidiscip. O., 44: 691-705. DOI: 10.1007/s00158011-0652-9

$\mathrm{Xu}, \mathrm{H}$. and P.H. Smets, 1996. Some strategies for explanations in evidential reasoning. IEEE Trans. Syst., Man, Cybern., 26: 599-607. DOI: $10.1109 / 3468.531907$

Yager, R.R., 1986. A modification of the certainty measure to handle subnormal distribution. Fuzzy Set Syst., 20: 317-324. DOI: 10.1016/S01650114(86)90042-4

Yager, R.R., J. Kacprzyk and M. Fedrizzi, 1994. Advances in the Dempster-Shafer Theory of Evidence. 1st Edn., John Wiley and Sons, New York, ISBN-10: 0471552488, pp: 597.

Yamada, K., 2001. Probability-possibility transformation based on evidence theory. Proceedings of the Joint 9th IFSA World Congress and 20th NAFIPS International Conference, Jul. 25-28, IEEE Xplore Press, Vancouver, BC., pp: 70-75. DOI: 10.1109/NAFIPS.2001.944229
Yang, J., H.Z. Huang, L.P. He, D. Wen and S.P. Zhu, 2011a. Risk evaluation in failure mode and effects analysis of aircraft turbine rotor blades using Dempster-Shafer evidence theory under uncertainty. Eng. Fail. Anal., 18: 2084-2092. DOI: 10.1016/j.engfailanal.2011.06.014

Yang, J., H.Z. Huang, Q. Miao and R. Sun, 2011b. A novel information fusion method based on Dempster-Shafer evidence theory for conflict resolution. Intell. Data Anal., 15: 399-411. DOI: 10.3233/IDA-2010-0474

Youn B.D., K.K. Choi, L. Du and D. Gorsich, 2005. Integration of possibility-based optimization to robust design for epistemic uncertainty. Proceedings of the 6th World Congresses of Structural and Multidisciplinary Optimization, (SMO’ 05), Brazil.

Youn, B.D. and K.K. Choi, 2004a. A New response surface methodology for Reliability-Based Design Optimization (RBDO). Comput. Struct., 82: 241256. DOI: 10.1016/j.compstruc.2003.09.002

Youn, B.D. and K.K. Choi, 2004b. Selecting probabilistic approaches for reliability-based design optimization. AIAA J., 42: 124-131. DOI: 10.2514/1.9036

Youn, B.D. and K.K. Choi, 2005. Enriched performance measure approach for reliability-based design optimization. AIAA J., 43: 874-884. DOI: $10.2514 / 1.6648$

Youn, B.D., 2005. Integrated framework for design optimization under aleatory and/or epistemic uncertainties using adaptive-loop method. Proceedings of the International Design Engineering Technical Conferences and Computers and Information in Engineering Conference, Sep. 24-28, California, USA., pp: 1253-1261. DOI: 10.1115/DETC2005-85253

Youn, B.D., K.K Choi and L. Du, 2004. Integration of reliability- and possibility-based design optimizations using performance measure approach. SAE I., 114: 247-256.

Youn, B.D., K.K. Choi and Y.H. Park, 2003. Hybrid analysis method for reliability-based design optimization. J. Mech. Des., 125: 221-232. DOI: 10.1115/1.1561042

Zadeh, L.A., 1978. Fuzzy sets as a basis for a theory of possibility. Fuzzy Set Syst., 1: 3-28. DOI: 10.1016/0165-0114(78)90029-5

Zhang, X., X.L. Zhang, H.Z. Huang, Z. Wang and S. Zeng, 2010b. Possibility-based multidisciplinary design optimization in the framework of sequential optimization and reliability assessment. Int. J. Innov. Comput. I., 11: 5287-5297. 
Zhang, X.D. and H.Z. Huang, 2010. Sequential optimization and reliability assessment for multidisciplinary design optimization under aleatory and epistemic uncertainties. Struct. Multidiscip. O., 40: 165-175. DOI: 10.1007/s00158-008-0348-y

Zhang, X.D., H.Z. Huang and H.W. Xu, 2010a. Multidisciplinary design optimization with discrete and continuous variables of various uncertainties. Struct. Multidiscip. O., 42: 605-618. DOI: 10.1007/s00158-010-0513-y

Zhou, J. and Z.P. Mourelatos, 2008. A sequential algorithm for possibility-based design optimization. J. Mech. Des., 130: 011001-011010. DOI: $10.1115 / 1.2803250$
Zhuang, Z.W., W.X. Yu and H. Wang, 2000. Information fusion and application in reliability assessment (in Chinese). J. Syst. Eng. Electron., 22: 75-80.

Zimmermann, H.J., 2000. An application-oriented view of modeling uncertainty. Eur. J. Oper. Res., 122: 190-198. DOI: 10.1016/S0377-2217(99)00228-3 